\title{
The supernova remnant population of the Small Magellanic Cloud
}

\author{
Pierre Maggi ${ }^{1}$, Miroslav D. Filipović ${ }^{2}$, Branislav Vukotić ${ }^{3}$, Jean Ballet ${ }^{4}$, Frank Haberl ${ }^{5}$, Chandreyee Maitra ${ }^{5}$, \\ Patrick Kavanagh ${ }^{6}$, Manami Sasaki ${ }^{7}$, and Milorad Stupar ${ }^{2}$

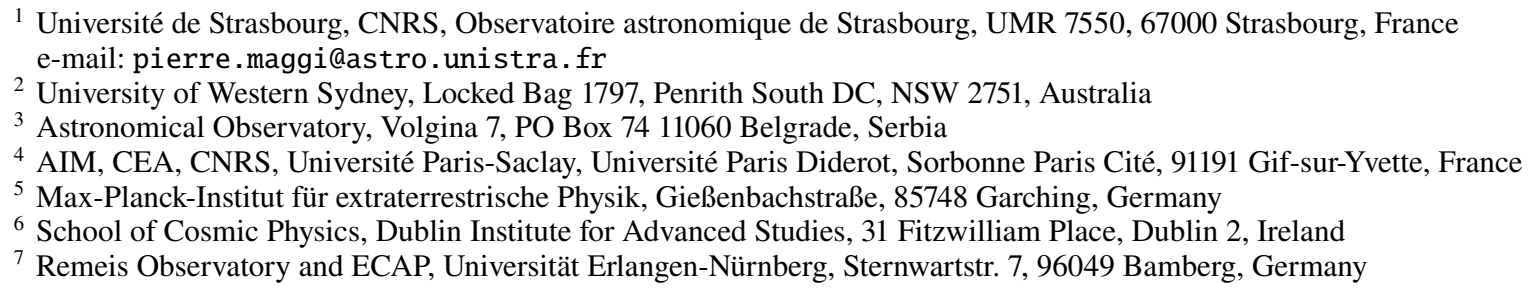

Received 27 August 2019 / Accepted 23 September 2019

\begin{abstract}
Aims. We present a comprehensive study on the supernova remnant (SNR) population of the Small Magellanic Cloud (SMC). We measured multiwavelength properties of the SMC SNRs and compare them to those of the Large Magellanic Cloud (LMC) population. Methods. This study combines the large dataset of XMM-Newton observations of the SMC, archival and recent radio continuum observations, an optical line emission survey, and new optical spectroscopic observations. We were therefore able to build a complete and clean sample of 19 confirmed and four candidate SNRs. The homogeneous X-ray spectral analysis allowed us to search for SN ejecta and $\mathrm{Fe} \mathrm{K}$ line emission, and to measure interstellar medium abundances. We estimated the ratio of core-collapse to type Ia supernova rates of the SMC based on the X-ray properties and the local stellar environment of each SNR.

Results. After the removal of unconfirmed or misclassified objects, and the addition of two newly confirmed SNRs based on multiwavelength features, we present a final list of 21 confirmed SNRs and two candidates. While no Fe K line is detected even for the brightest and youngest SNR, we find X-ray evidence of SN ejecta in 11 SNRs. We estimate a fraction of 0.62-0.92 core-collapse supernova for every supernova (90\% confidence interval), higher than in the LMC. The difference can be ascribed to the absence of the enhanced star-formation episode in the SMC, which occurred in the LMC 0.5-1.5 Gyr ago. The hot-gas abundances of $\mathrm{O}, \mathrm{Ne}, \mathrm{Mg}$, and Fe are 0.1-0.2 times solar. Their ratios, with respect to SMC stellar abundances, reflect the effects of dust depletion and partial dust destruction in SNR shocks. We find evidence that the ambient medium probed by SMC SNRs is less disturbed and less dense on average than in the LMC, consistent with the different morphologies of the two galaxies.
\end{abstract}

Key words. ISM: supernova remnants - Magellanic Clouds - ISM: abundances - supernovae: general - X-rays: ISM

\section{Introduction}

A fraction of stars end their lives in powerful supernova (SN) explosions (e.g. Alsabti \& Murdin 2017, and references therein). This is the case after core-collapse (CC) for some of the most massive stars (zero age main sequence mass $\gtrsim 8 \quad M_{\odot}$ ), and through the thermonuclear disruption of the $\mathrm{CO}$ core of a white dwarf (the so-called type Ia SNe), which is possibly ignited when the Chandrasekhar mass is reached via accretion, or during the merger of a double white dwarf binary. Both types of SNe release large quantities of freshly-produced elements from light $\alpha$-group elements $(\mathrm{O}, \mathrm{Ne}$, and $\mathrm{Mg}$ ) to intermediate-mass elements ( $\mathrm{Si}$ and $\mathrm{S}$ ) and heavier $\mathrm{Fe}$-group elements (Ti, $\mathrm{Cr}, \mathrm{Fe}$, and $\mathrm{Ni}$ ), produced during thermostatic nuclear burning and in the final, explosive nucleosynthesis episode. Together with stellar winds and neutron star mergers, SNe are responsible for the enrichment and chemical content of the Universe (Nomoto et al. 2013; Thielemann et al. 2017).

Furthermore, the tremendous energy release of an SN $\left(\sim 10^{51} \mathrm{erg}\right)$ is transferred to the surrounding interstellar medium (ISM). The object created in the ISM by an $\mathrm{SN}$ is called a supernova remnant (SNR). The $\mathrm{SN}$ ejecta launched at velocities greater than $10^{4} \mathrm{~km} \mathrm{~s}^{-1}$ drive shock waves in the ambient medium, heating the ISM and ejecta up to X-ray emitting temperatures (Vink 2012). Cosmic rays (particles) are accelerated at the shock front where the magnetic field is turbulent, and electrons with energies up to $100 \mathrm{TeV}$ (for the youngest SNRs) emit synchrotron radiation from radio to X-rays (Koyama et al. 1995). Optical line emission can arise mostly from charge exchange and collisional excitation of neutrals at fast shocks (Ghavamian et al. 2001), shock-ionised material, or radiative cooling if the conditions are conducive (Cox \& Daltabuit 1971).

Remnants remain visible for several $10^{4} \mathrm{yr}$, as opposed to a few hundred days for their parent SNe. Consequently, the SNR population of a galaxy collectively holds precious information about the dozen or hundreds of $\mathrm{SNe}$ that recently exploded within it. For instance, the ratio between the rates of $\mathrm{SNe}$ of each type (CC vs. type Ia) can in principle be recovered. The morphologies of individual SNRs can be linked to asymmetries, either intrinsic to the explosion or coming from its surrounding ISM (Wongwathanarat et al. 2013; Lopez 2014). Additionally, the abundances of newly-synthesised ejecta constrain details about both stellar evolution and explosion physics (Chieffi \& Limongi 2017; Leung \& Nomoto 2018). 
The many SNe exploding in a galaxy are the main source of energy of its ISM, in the form of kinetic energy, turbulence, and cosmic ray acceleration (Mac Low \& Klessen 2004). They offer a mode of star formation regulation, as the combined shocks of many $\mathrm{SNe}$ can launch galactic winds which expels gas. Heated to temperatures $T>10^{6} \mathrm{~K}$, the dominant elements of the ISM (C, N, O, Ne, Mg, Si, S, Fe) emit many lines in the X-ray band which can be used to infer their abundances. Therefore, studies of populations of SNRs in a galaxy can reveal key information on the SNe themselves and can be used to probe the host galaxy.

The Milky Way population currently contains about 300 SNRs (Green 2019). The studies regarding them are hampered by large distance uncertainties and line-of-sight confusion and crowding, which prevent accurate comparison of objects. The strong interstellar absorption towards most of these sources in the Galactic plane is even more problematic, particularly for X-rays as the important $0.5-2 \mathrm{keV}$ energy band can be completely masked for $N_{\mathrm{H}}>10^{22} \mathrm{~cm}^{-2}$. Despite larger distances, external galaxies are therefore better suited to SNR population studies. The SNRs of several galaxies in the Local Group (M 31, M 33, Sasaki et al. 2012; Long et al. 2010; Galvin \& Filipovic 2014; Garofali et al. 2017) and beyond (Payne et al. 2004a; Chomiuk \& Wilcots 2009; Leonidaki et al. 2010; Millar et al. 2011, 2012; Pannuti et al. 2011, 2015; O'Brien et al. 2013; Long et al. 2014; Galvin et al. 2014; Yew et al. 2018) have been studied at various wavelengths. Closer to us, our Galactic neighbours the Large and Small Magellanic Clouds (LMC, SMC) provide excellent benchmarks for the study of star-forming galaxies. At only $50 \mathrm{kpc}$ and $60 \mathrm{kpc}$, respectively (Pietrzyński et al. 2019; Hilditch et al. 2005; Graczyk et al. 2014), they are close enough that we can detect and spatially resolve SNRs from radio to X-ray wavelengths, and are located behind only a moderate Galactic foreground $\left(N_{\mathrm{H}} \lesssim\right.$ a few $10^{20} \mathrm{~cm}^{-2}$, Dickey \& Lockman 1990).

In the LMC, our knowledge about the SNR population increased over time with radio, optical, and X-ray observations. In Maggi et al. (2016, hereafter MHK16), a comprehensive X-ray study of the LMC SNRs was conducted, taking advantage of the coverage of a large fraction of the SNR population ( $\sim 60$ objects) with the XMM-Newton X-ray observatory during targeted observations and the extensive survey of the LMC (PI: F. Haberl). The radio counterpart to that study, also presenting 15 further SNR candidates, was published in Bozzetto et al. (2017, hereafter B17).

Likewise, the SNR population of the SMC benefitted greatly from successive X-ray surveys. The first X-ray imaging survey of the SMC (Seward \& Mitchell 1981) revealed six SNRs and an equal number of candidates (Inoue et al. 1983), although their classifications were still uncertain in later analyses (Bruhweiler et al. 1987; Wang \& Wu 1992). The extensive pointed survey of the SMC with ROSAT led to the detection of X-ray emission from 17 SNRs or SNR candidates at the time (Kahabka et al. 1999; Haberl et al. 2000). Observations by the Japanese satellite ASCA did not find new SNRs due to a mediocre pointspread function but allowed the first X-ray spectral analysis of SMC SNRs thanks to the better energy resolution of ASCA gas imaging spectrometer Yokogawa et al. (2000).

In this work, we attempt to provide the most comprehensive study of the SNR population of the SMC. As the sample is about three times smaller than in the LMC, we combine in a single work both the X-ray and radio-continuum analyses, together with archival optical emission line data and new optical spectroscopy. Previous similar studies were more limited in their scope. van der Heyden et al. (2004) analysed
XMM-Newton data for 13 SMC SNRs. We expand considerably on this work, using the much larger body of XMM-Newton observations accumulated since, a larger population augmented with newly-discovered objects (e.g. Filipović et al. 2008), and, as already mentioned, a multiwavelength approach including deep radio and optical surveys.

The paper is organised as follows: in Sect. 2 we describe the XMM-Newton, radio, and optical observations used and how the data were reduced. The X-ray imaging and spectral analyses are detailed in Sect. 3. Our results are presented and discussed in Sect. 4, starting with the final sample and rejected objects in Sects. 4.1 and 4.2, respectively, followed by the X-ray spectral properties of SMC SNRs (Sect. 4.3). Candidates and confirmed SNRs are discussed individually in Sects. 4.4.2 and 4.5. We then measure the gas-phase abundances of the SMC ISM and the ratio of CC to type Ia SNe (Sects. 4.6 and 4.7), discuss the radio properties, size, and morphology of SNRs in both Magellanic Clouds (MCs; Sect. 4.8), and probe the 3D spatial distribution of SNRs within the SMC (Sect. 4.9). Our findings are summarised in Sect. 5.

\section{Observations and data reduction}

\subsection{X-ray data}

There are upwards of 120 XMM-Newton observations of the SMC. In this work, all observations useful for imaging and/or spectroscopic purposes were included. Lists of observation IDs (ObsIDs) sorted by off-axis angle and exposure time were compiled for each SNR. Thanks to the compactness of the SMC and its dense coverage with $X M M-N e w t o n$, all SNRs have multiple observations available. That number ranges from two (for two SNRs) to 38 for four of them. Those highly covered are those in the field of view of SNR 1E 0102.2-7219 (including itself), which is used as a calibration source (Plucinsky et al. 2017) and thus monitored frequently.

All data were processed with the "MPE pipeline", used for XMM-Newton surveys of M31 (Pietsch et al. 2005; Stiele et al. 2011), M33 (Pietsch et al. 2004; Misanovic et al. 2006), and the SMC (Haberl et al. 2012b). A summary of the important steps of the pipeline was given in MHK16. The difference with Haberl et al. (2012b) is that data were reprocessed with version 16.0.0 of the XMM-Newton Science Analysis Software ${ }^{1}$. The resulting event lists and associated good time interval files (gti, one file per detector) were used as the primary source for subsequent analysis.

\subsection{Radio continuum data}

In a similar manner as in B17 where the LMC radio continuum sample were investigated, we used all available radio continuum data described in various surveys to date (Filipović et al. 1997, 1998, 2002, 2005; Payne et al. 2004b, 2007; Reid et al. 2006; Wong et al. 2011a,b, 2012a,b; Crawford et al. 2011). Some earlier radio continuum studies of selected SMC SNRs were shown in Filipović et al. (2008), Owen et al. (2011), Haberl et al. (2012c), Crawford et al. (2014), Maitra et al. (2015). Where possible, our study improves upon these previous SMC SNR studies. We also examine Australia Telescope Online Archive to search for in depth coverage of SNRs studied here. Apart from the ATCA (and Parkes) radio telescope, we make use of MWA observations as described in For et al. (2018) as well as the Australia Square Array Pathfinder (ASKAP) Early Science Project on the

SAS, http://xmm.esac.esa.int/sas/ 

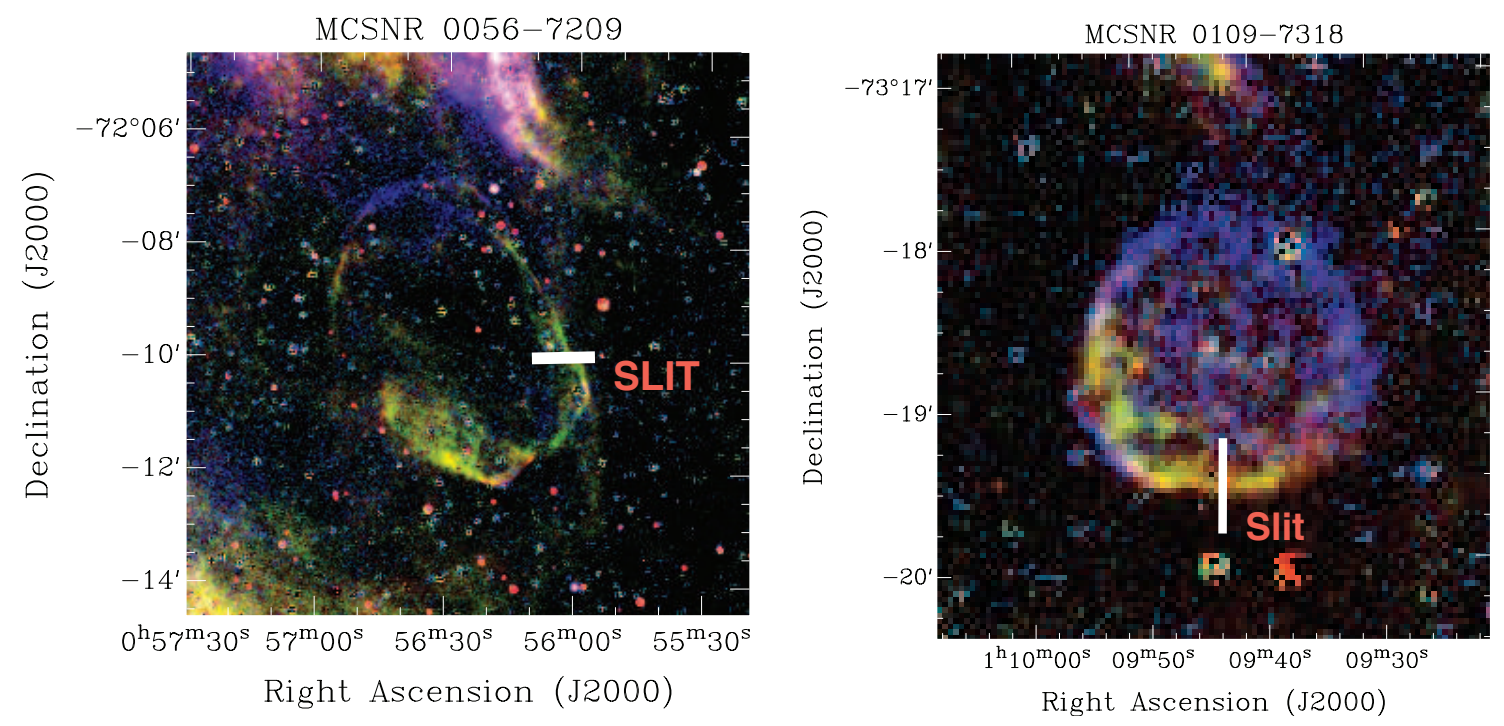

Fig. 1. Left: MCSNR candidate J0056-7209 on a composite MCELS image (R, G, B = [S II], H $\alpha$, and [O III], respectively). On the east and west side of the ellipse, fragmented filaments typical of older supernova remnants are clearly seen. The white bar shows the position of the WiFeS spectrograph slit. The spectrograph slit is actually a combination of $25 \times 1^{\prime \prime}$ wide adjacent slits each, repeated 36 times to yield an effective $25 \times 36^{\prime \prime}$ field of view on the sky. Right: same as left for MCSNR candidate J0109-7318. Compared with J0056-7209, this one exhibits more fragmented filaments creating [S II] and $\mathrm{H} \alpha$ arcs also common in morphological structures of old supernova remnants.

SMC (Joseph et al. 2019). Typical data reduction procedures as described in above papers were used.

Since our SMC SNR sample is morphologically diverse and comes from heterogeneous datasets, various approaches (and initial parameters) were employed in order to measure the best SNR flux densities and their errors. Namely, we used the MIRIAD (Sault et al. 1995) task imfit and imsum to extract integrated flux density (and error), extensions (diameter/axes) and position angle for each radio-detected SNR. For cross checking and consistency, we also used AEGEAN and found no significant difference in integrated flux density estimates.

We used two methods: For SNRs which are known unresolved sources (for example 1E 0102.2-7219) we use simple Gaussian fitting which produced the best result. The second approach was applied to all resolved SNRs. For those, we measured their local background noise $(1 \sigma)$ and carefully select the exact area of the SNR. We then estimated the sum of all brightnesses above $5 \sigma$ of each individual pixel within that area and converted it to SNR integrated flux density following Findlay (1966, Eq. (24)). We also made corrections for an extended background where applicable, that is, for sources where nearby extended object such as H II region(s) is evident. However, for the most of our SMC SNRs this extended background contribution is minimal.

The radio spectral index $(\alpha)$ based on at least two flux density $\left(S_{v}\right)$ measurements is then estimated as defined via $S_{v} \propto v^{\alpha}$. The validation of our entire SMC radio sources sample including SNRs are presented in Joseph et al. (2019). There, we argue that the measured radio flux densities and corresponding errors are well below $10 \%$. We also emphasis that the small radio spectral index error estimates (see Table A.1; Col. 11) are statistical errors which are based on a true flux density error estimates.

\subsection{Optical observations of SMC SNRs candidates}

In our optical search for the SMC SNRs we initially used the Magellanic Clouds Emission Line Survey (MCELS; see Pellegrini et al. 2012). In this survey which covered optical wavebands of [O III] at $5007 \AA, \mathrm{H} \alpha$ at $6563 \AA$ and [S II] at $6716 / 6731 \AA$, we found two new objects (see Fig. 1) whose morphological structures are typical of SNRs. This was the initial motivation for us to go further and obtain optical spectra of these objects, named candidates MCSNR J0056-7209 and MCSNR J0109-7318, and try to confirm their nature.

Spectral observations were undertaken on June 8, 2015 (see Table 2), using the Wide-Field Spectrograph (WiFeS) on the 2.3-m telescope of the Mount Stromlo and Siding Spring Observatory (MSSSO). The WiFeS spectrograph is an image slicer and behaves as an integral field unit (IFU) providing spatiallyresolved spectroscopy (see details in Dopita et al. 2007). The final result, after complex data reduction of WiFeS observations, is a cube with RA, Dec and wavelength as third dimension. From that cube, we can generate $1 \mathrm{D}$ spectra. WiFeS consists of twenty-five $1^{\prime \prime}$ wide adjacent slits which are each $36^{\prime \prime}$ in length to yield an effective $25 \times 36^{\prime \prime}$ on the sky. As our granted observational night definitely was not photometric, we performed observations only in the red part of the spectrum between 5700 and $7000 \AA$ using the R7000 grating with 1200 lines $\mathrm{mm}^{-1}$. In addition, due to the non-photometric night we could neither apply observations of spectrophotometric standard stars to get real line fluxes (but used simple counts) nor estimate the true extinction.

\section{Data analysis}

\subsection{X-ray imaging}

Images were created with a pixel size of $2^{\prime \prime} \times 2^{\prime \prime}$, using single to quadruple-pixel events (PATTERN $=0-12)$ with FLAG $=0$ from the MOS detectors. Single and double-pixel events (PATTERN = $0-4)$ from the pn detector with (FLAG \&\& $0 \times f 0000$ ) $=0$ (i.e. including events next to bad pixels or bad columns) were used. To avoid the higher detector noise contribution from the doublepixel events below $500 \mathrm{eV}$, only single-pixel events were selected at these low energies. Exposure maps taking into account the energy-dependent telescope vignetting were produced with the 
task eexpmap. Out-of-time (OoT) images were created from the EPIC-pn OoT event lists, scaled by the corresponding OoT fraction $f_{\mathrm{OoT}}{ }^{2}$, and subtracted from the corresponding source+background images.

Images and exposure maps were extracted in various energy bands for all three cameras. The set of energy bands was tailored to the thermal spectrum of SNRs: a soft band from 0.3 to $0.7 \mathrm{keV}$ includes strong lines from oxygen; a medium band from 0.7 to $1.1 \mathrm{keV}$ comprises Fe L-shell lines as well as K-shell lines from $\mathrm{Ne}$ IX and Ne X; and a hard band (1.1-4.2 keV) includes $\mathrm{K}$ lines from $\mathrm{Mg}, \mathrm{Si}, \mathrm{S}, \mathrm{Ca}, \mathrm{Ar}$, and possibly non-thermal continuum.

The detector background was subtracted from the images. We used filter wheel closed (FWC) data, obtained with the detectors shielded from astrophysical and soft-proton backgrounds. FWC observations are collected several times per year as part of the XMM-Newton calibration efforts and made available by the XMM-Newton Science Operations Centre ${ }^{3}$. The instrumental background contribution $f_{\mathrm{FWC}}$ to the science image is estimated from the count rate in the detector corners for each instrument individually, as they are always shielded from the X-ray telescopes. The FWC images were scaled by $f_{\mathrm{FWC}}$ and removed from the science image to create the background-subtracted image. Only FWC exposures in full-frame mode are available for MOS detectors, excluding all other modes from our analysis.

We combined all suitable observations of an SNR to produce an image centred on the source. In each band, we merged the images from pn and both MOS. The smoothing of the combined images was done in adaptive mode with the SAS task asmooth. It calculates a library of Gaussian kernels such that the resulting images reach a minimum (Poissonian) signal-to-noise ratio of 5 everywhere. Regions of good statistics (e.g. bright sources) are smoothed with a small kernel, whereas fainter regions are more thoroughly smoothed. The minimum kernel size for adaptive smoothing is either $10^{\prime \prime}$ or $20^{\prime \prime}$, depending on the available data and the surface brightness of the SNR under investigation. Only MCSNR J0104-7201 (1E 0102.2-7219) was smoothed with a smaller kernel of $6^{\prime \prime}$ owing to its small size and excellent photon statistics available. We divided the combined image by the corresponding vignetted and smoothed exposure map. The combined exposure map was produced by weighting the MOS exposure maps with a factor of 0.4 relative to $\mathrm{pn}$, to account for the lower effective area. The smoothing of the exposure map is done with the same template of kernels as the for the images. The resulting composite images (soft-medium-hard bands) are shown in Appendix B with radio and optical features.

\subsection{X-ray spectra}

We follow the spectral analysis method described extensively in MHK16: we simultaneously fit source and background spectra (hereafter SRC and BG), where the latter is explicitly modelled rather than subtracted. This is critical for the analysis of faint extended sources such as SNRs in the SMC. We correct the event lists for vignetting with the SAS task evigweight prior to extraction. This accounts for the energy-dependent effective area variation across the extents of SNRs and background regions. The redistribution matrices are produced by the SAS task rmfgen, and the ancillary response files by arfgen. The latter is used in unvignetted mode (equivalent to a flat detector map), returning the on-axis effective area, because the vignetting is already corrected event-wise.

\footnotetext{
2 Values taken from the XMM-Newton Users Handbook.

3 http://www. cosmos.esa.int/web/xmm-newton/

filter-closed
}

We use the same event pattern for spectra as for imaging. We use the spectral-fitting package XSPEC (Arnaud 1996) version 12.9.0e, with spectra rebinned with a minimum of 25 counts to allow the use of the $\chi^{2}$-statistic. Interstellar absorption is reproduced by the photoelectric absorption model phabs in XSPEC (or vphabs, where the prefix " $\mathrm{v}$ " indicates that abundances can vary), using cross-sections set to those of Balucinska-Church \& McCammon (1992).

The extraction regions for SRC and BG spectra are defined manually, usually guided by the X-ray contours. Simple shapes (circles, ellipses) are preferred, but an arbitrary shape (e.g. polygonal region) is also used if required. Point sources detected during the pipeline data reduction with the task edetectchain are excluded. Details of the definition of extraction regions are given in MHK16.

When defining extraction regions, we also screen out observations not suited for spectroscopy (that might have been used for imaging). For instance, we do not use the shorter observations if many longer exposures are available, those at large off-axis angle, and those where the SNR is only partially in the field of view (i.e. over the detector edges). In the end, a variety of spectra combination can be found, from pn/MOS1/MOS2 data from a single observation (e.g. for MCSNR J0051-7321), up to a combination of spectra from six observations (e.g. for MCSNR J0058-7217, fitting 16 spectra simultaneously).

Spectra extracted from FWC data at the same detector positions as the SRC and BG regions are used to fit the instrumental background model. It comprises electronic noise and particleinduced background, as described in Kuntz \& Snowden (2008); Sturm (2012); and MHK16. The instrumental background is not vignetted, and the vignetting-weighting process used on science data distorts its spectrum, particularly at high-energy where the vignetting effect is the strongest. We correct for this by including an ad-hoc multiplicative spline function in the model of the instrumental background. The best-fit models are used in subsequent fits (including astrophysical signal) with no free parameter, as the instrumental background averaged in the FWC dataset matches generally well with the one in the SNR spectra.

One component of the background is the SMC diffuse emission. This was studied by Sturm (2012), who modelled the diffuse emission with a thermal model :

$S_{\mathrm{SMC} \mathrm{diff}}=\operatorname{phabs}\left(N_{\mathrm{H}}^{\mathrm{Gal}}\right) \times \operatorname{vphabs}\left(N_{\mathrm{H}}^{\mathrm{SMC} *}, 0.2 Z_{\odot}\right) S_{\mathrm{apec}}^{\mathrm{SMC}}$,

where $S_{\text {apec }}^{\mathrm{SMC}}$ is the emission from an apec model ${ }^{4}$ at temperature $k T^{\mathrm{SMC}}$ and normalisation $N^{\mathrm{SMC}}$. The foreground column density $N_{H}^{\mathrm{Gal}}$ at the location of each analysed source is taken from the

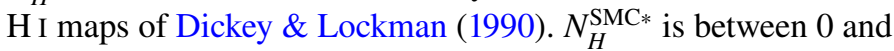
$N_{H}^{\mathrm{SMC}}$, the total line-of-sight column density through the SMC (Stanimirovic et al. 1999). Parameters of SMC diffuse emission are given by Sturm (2012) in a grid of 240 boxes (size of $9^{\prime} \times 9^{\prime}$ ), containing all but one SNR (MCSNR J0127-7333). The significance of the diffuse emission component is higher than $3 \sigma$ in all the boxes hosting an SNR, except for MCSNR J0040-7336, where the diffuse emission is very faint (the normalisation of the diffuse component generally correlates with its significance). For completeness, we included the diffuse SMC emission in the background model of MCSNR J0041-7336, as it does not affect the rest of the fit much.

The remaining astrophysical X-ray background (AXB) comprises Galactic and extragalactic components: unabsorbed

$\overline{4}$ Using AtomDB 3.0, http : //www . atomdb.org/index .php 
thermal emission from the local hot bubble and an absorbed twotemperature plasma emission from the halo. The cosmic X-ray background is modelled as a power law with a photon index $\Gamma$ of 1.41 (De Luca \& Molendi 2004). The final model for the AXB is:

$$
\begin{aligned}
S_{\mathrm{AXB}}= & S_{\text {apec }}^{1}+\operatorname{phabs}\left(N_{\mathrm{H}}^{\mathrm{Gal}}\right)\left(S_{\mathrm{apec}}^{2}+S_{\mathrm{apec}}^{3}\right. \\
& \left.+\operatorname{vphabs}\left(N_{\mathrm{H}}^{\mathrm{SMC}}, 0.2 Z_{\odot}\right) N_{\mathrm{CXB}} E^{-\Gamma}\right) .
\end{aligned}
$$

The temperatures of the thermal components $\left(k T^{1}=108 \mathrm{eV}\right.$, $k T^{2}=36 \mathrm{eV}$, and $k T^{3}=247 \mathrm{eV}$ ) and normalisations are taken from Sturm (2012), where this model was fit on observations around the main SMC field. Thus, it gives a fair representation of Galactic foreground and extragalactic background towards the SMC.

Another non-X-ray background component is the soft proton contamination (SPC), which we model following the prescription of Kuntz \& Snowden (2008). The SPC parameters are highly time-variable and position-dependent, so they were different for each instrument and observation.

The final background model (FWC $+S_{\mathrm{SMC} \text { diff }}+S_{\mathrm{AXB}}+$ $\mathrm{SPC}$ ) is fit to the BG spectra prior to fitting source emission. In most cases good fits are obtained with only a constant renormalisation factor for $S_{\mathrm{SMC} \text { diff }}$ and $S_{\mathrm{AXB}}$. For five SNRs, the background model fit was significantly improved by varying the parameters of the SMC diffuse emission $\left(N_{\mathrm{H}}^{\mathrm{SMC} *}\right.$ and $\left.k T^{\mathrm{SMC}}\right)$ or the normalisations of the various XRB components. This is likely due to variations of the X-ray background or diffuse emission on small angular scales.

\section{Results and discussion}

\subsection{Final sample}

We searched all available optical, radio and X-ray surveys in order to secure the most complete population of the SMC SNRs. The number of confirmed SNRs in the SMC is currently at 19 (see Table A.1). Sources previously classified as SNRs which were not included in the final sample are discussed in Sect. 4.2. In addition, we list in this work two SMC SNR candidates which are presented here for the first time (Table A.2). These new SNR candidates are given the identifiers MCSNR candidate J0106-7242 and MCSNR candidate J0109-7318, and join two other candidates, MCSNR candidate J0056-7209 and MCSNR candidate J0057-7211, which were presented in Haberl et al. (2012b). Primarily, we classified the 4 SMC SNR candidates based on the well established criteria described in Filipović et al. (1998). For more details, see Table A.2 and Sect. 4.5.

The extent of all 23 SNRs and SNR candidates is primarily measured using MCELS images, with some additional information obtained via our various radio images as well as Chandra, XMM-Newton, or ROSAT surveys when needed. Where possible, we determined SNR diameters from the highest resolution image available including optical and X-ray images. We estimated that the error in diameter is smaller than $2^{\prime \prime}$ or $\sim 0.58 \mathrm{pc}$. We also found that our diameters shown here could be different at different wavebands (usually within $\sim 10 \%$ ) as it was the case in the LMC (B17; MHK16). All SMC SNRs and SNR candidates' radio flux density measurements are shown here for the first time and their associated errors are well below $10 \%$. For the sake of consistency, we assumed a common distance of $60 \mathrm{kpc}$ to all sources for our measurements and derived properties. The expected dispersion along the line-of-sight due to the depth of the SMC (see Sect. 4.9) is likely higher than the uncertainties of e.g. angular sizes and fluxes. Because of their very low surface brightness we could not detect radio emission from two of the 4 SMC SNR candidates. Also, we could not measure the flux density of MCSNR J0103-7201 (Haberl et al., in prep.) as it is a very weak radio source with a very thin (but distinguishable in our high sensitivity ATCA-CABB observations) shell, which overlaps with the neighbouring massive H II region DEM S103 (see Fig. B.6). Therefore, Table A.1 is a compilation of our own measurements as well as those of other papers for this well established sample of the SMC SNRs.

\subsection{Objects not included}

We present here a list of objects previously classified as SNR or SNR candidate that, upon closer scrutiny and in light of the new datasets in radio and X-rays, can no longer be bona-fide SNRs. Most of these objects were originally suggested to be (possible) SNRs based on a single feature (e.g. radio, X-rays). None were later confirmed by a multi-wavelength detailed study, although they have been since included in SNR samples and compilations. This attempt at "cleaning" the literature will be beneficial for population studies, making sure they do not include such unrelated objects (as e.g. in Badenes et al. 2010; Auchettl et al. 2019), which is likely to introduce biases.

\subsubsection{N S19 ([FBR2002] J004806-730842)}

From the ATCA radio catalogue of Filipović et al. (2005), this source is the very confused LHA $115-\mathrm{N} 19$ (hereafter N 19) $\mathrm{H}$ II complex. Radio emission thus includes both thermal contribution from the HII region and non-thermal (synchrotron) emission by the nearby three genuine SNRs in that area (see Sect. 4.5.2 and Fig. B.2). The new ASKAP data do not resolve this source into a shell, as expected for a true SNR. Very faint soft X-ray emission was noted in the SMC X-ray survey (Haberl et al. 2012b), but it is to the north-east of the radio source and from a larger region, itself surrounded by small optical features. We conclude that the radio features are likely just thermal emission from optical nebulosities within N 19, while the large size, soft and very faint X-ray diffuse emission is akin to a superbubble, resembling e.g. LHA 120-N 51D in the LMC (Bomans et al. 2003; Yamaguchi et al. 2010).

\subsubsection{SNR B0045-73.3}

Like N S19, it is a radio source in the N 19 complex (Filipović et al. 2002), but the ASKAP emission is not resolving a shell, instead highlighting optical nebulosity of the $\mathrm{H}$ II region with interior [O III] emission, a better indication of photoionisation. Some X-ray diffuse emission is also seen (Haberl et al. 2012b), but again it does not match the radio or optical features. This could be just hot gas seen within N 19.

\subsubsection{NS21 ([FBR2002] J004748-731727)}

It is an unresolved radio source (Filipović et al. 2002) with no X-ray emission (down to $\sim 10^{-14} \mathrm{erg} \mathrm{cm}^{-2} \mathrm{~s}^{-1} \operatorname{arcmin}^{-2}$, Haberl et al. 2012b). The small optical nebulosities at that position point towards the radio source being thermal emission from photoionised $\mathrm{H}$ II regions.

\subsubsection{IKT7 ([HFP2000]424)}

It was suggested as an SNR candidate based on Einstein observatory hardness ratios (Inoue et al. 1983). Later this source was 
confidently identified via its $172 \mathrm{~s}$ pulsations as the Be/X-ray binary AX J0051.6-7311 (Haberl \& Pietsch 2004). The absence of optical, radio, or diffuse X-ray emission proves that this is not an SNR, a conclusion already mentioned in Haberl et al. (2012b).

\subsubsection{DEM S130 ([FBR2002] J010539-720341)}

This is a radio source around a bright emission-line star (LHA 115-N 78C). DEM S130 designates the compact HII region which likely is the source of thermal radio emission. There is no X-ray emission despite this area being the deepest covered with XMM-Newton, in the field of view of MCSNR J0104-7201.

\subsubsection{LHA 115-N 83C}

There are no studies of this radio candidate (Filipović et al. 2005) in the south-east of the SMC, which shows no X-ray emission. It is likely a compact $\mathrm{H}$ II region (photoionised, filled with [O III] emission) within the LHA 115-N 83 (=NGC 456) complex.

\subsection{X-ray spectral properties of SMC SNRs}

\subsubsection{General properties}

The results of the spectral analysis for the SMC sample are given in Table A.3. Only MCSNR J0103-7201 and J0104-7201 have not been included (see Sect. 4.5).

The X-ray analysis opens the possibility to follow the evolution of thermal energy $(P=n k T)$ of the SNR, whose volume integral we expect to be at most $0.47 E_{\mathrm{SN}}$ in the Sedov phase, as function of the size $R$. A proxy for the density is obtained from the emission measure as $n_{\mathrm{H}}=f^{-1} 0 \sqrt{\mathrm{EM} / 1.2 \mathrm{~V}}$, with a factor 1.2 for a fully ionised plasma, and a filling factor $f<1$. The volume was calculated assuming an ellipsoid shape with minor and major axes, and a third axis assumed to be in between these values. We propagate the uncertainty of this assumption in our calculation. To avoid having the size $R$ entering both $x-$ and $y$-axes (because $n \propto R^{-1 / 3}$ ), we plot in Fig. 2 the product $P \times \sqrt{V} \equiv f^{-1} \sqrt{\mathrm{EM}} \times k T$ as a function of average diameter in pc. For multiple-component spectra, we used the sum of all EM, and the EM-averaged temperature. Lines of constant energy $f \times E_{\mathrm{SN}} \propto R^{-3 / 2}$ are overplotted. We show the results for the SMC population (this work) and the LMC SNRs (MHK16). There is no tight downward correlation. The scatter reflects at least some intrinsic variation in explosion energy, but most likely it is due to part of these SNRs having entered the radiative phase (in many cases indicated by prominent optical emission), which lowered their internal energy.

\subsubsection{Fe K emission}

$\mathrm{Fe} \mathrm{K}$ lines were shown to be a valuable tool to distinguish type Ia from CC SNRs (Yamaguchi et al. 2014, but see caveats in Maggi \& Acero 2017). However, no Fe $\mathrm{K}$ emission has been reported from SMC SNRs in the literature or found in our analysis. We examined the high-energy emission of MCSNR J0104-7201 (1E 0102.2-7219), the brightest SNRs in our sample with the hottest plasma, to assess the presence of faint $\mathrm{Fe} \mathrm{K}$ emission or derive upper limits. With a simple Bremsstrahlung continuum, we fit the spectrum above $3 \mathrm{keV}$, a band devoid of strong line besides $\mathrm{Fe} \mathrm{K}$, as $\mathrm{Ar}$ and $\mathrm{Ca}$ lines ( 3-4.5 keV) have much smaller equivalent widths. Then, a Gaussian line with zero width is added at a centroid energy ranging from $6.4-6.7 \mathrm{keV}$ and the $3 \sigma$ uncertainty on the line

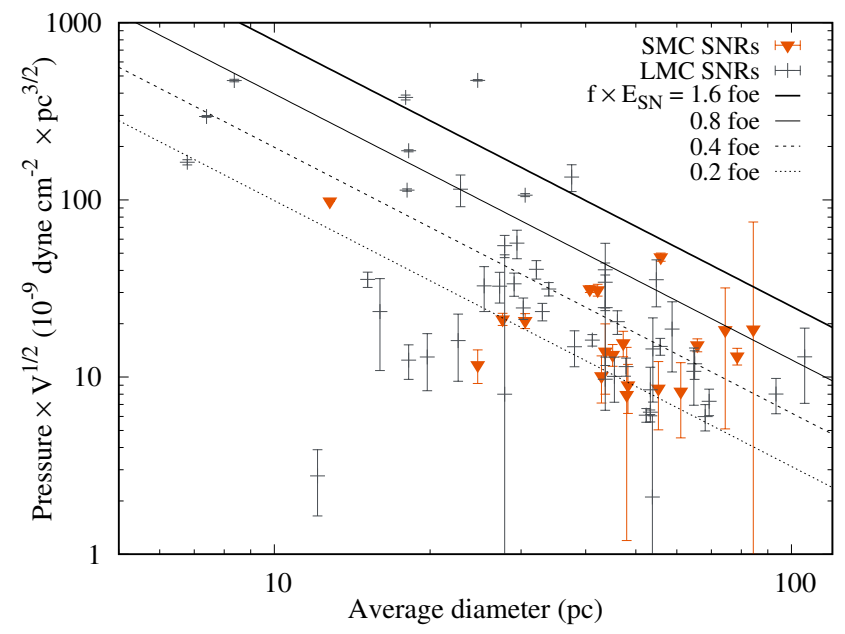

Fig. 2. $P \times V^{1 / 2}$ of SNRs in SMC (orange triangles, this work) and LMC (grey plus signs, MHK16) as a function of their size. Lines of constant SN energy (times filling factor $f$, see text in Sect. 4.3.1) are overplotted in units of $10^{51} \mathrm{erg} \equiv 1$ foe.

normalisation is calculated. Over the tested centroid range the error bars always cross zero, consistent with no detection.

The upper limit was at most $0.9 \times 10^{-6} \mathrm{ph} \mathrm{s}^{-1} \mathrm{~cm}^{-2}$. In the whole sample of Fe K emitting SNRs, only two Galactic sources (Cas A and W49B) and two LMC sources (N103B and N132D) would have fluxes above this limit at the SMC distance and would potentially be detectable. At an age of about $2000 \mathrm{yr}$ (1700 to 2600 yr, Finkelstein et al. 2006; Xi et al. 2019), the expansion measurement and explosion modelling suggest that the remaining unshocked ejecta in MCSNR J0104-7201 include most of the iron produced in the SN (Xi et al. 2019), explaining the lack of Fe emission. All other SMC SNRs are more evolved and their X-ray temperatures are likely too low to promote $\mathrm{Fe} \mathrm{K}$ line emission, in addition to being fainter overall, further preventing detection with existing instruments.

\subsubsection{Detection of $\mathrm{SN}$ ejecta}

Supernova ejecta can be revealed in X-ray spectra by high abundances of metals, significantly above the average SMC abundance (0.1-0.2 times solar) or even super-solar. Since there are stark contrasts in the nucleosynthesis yields of thermonuclear and $\mathrm{CC} \mathrm{SNe}$, ratios of $\mathrm{O}, \mathrm{Ne}$, and $\mathrm{Mg}$ to $\mathrm{Fe}$ abundances can provide valuable information as to the type of progenitor of a given SNR.

Using the results of our spectral analysis, we flag in Table 1 the SNRs where the $\mathrm{X} / \mathrm{Fe}$ abundance ratios (where $\mathrm{X}$ is $\mathrm{O}, \mathrm{Ne}$, $\mathrm{Mg}$, or $\mathrm{Si}$ ) are significantly higher or lower than the average SMC value (Russell \& Dopita 1992). The three SNRs with low $\mathrm{X} / \mathrm{Fe}$ ratios are those akin to several evolved LMC SNRs with iron-rich, centrally-bright emission (MHK16). In some cases an elevated $\mathrm{Fe}$ abundance or sometimes a pure $\mathrm{Fe}$ component are needed to fit the spectra, leading to their classification as "low $\mathrm{X} / \mathrm{Fe}$ " cases, even if the low-Z elements were not left free. We make use of these flags in the typing of SMC SNRs (Sect. 4.7).

\subsection{New SMC SNR candidates}

\subsubsection{Optical spectroscopy}

Examination of WiFeS 1D spectra (see Fig. 3) shows all main lines typical of old SNRs. They show lines of [N II] at 6548 and 
Table 1. SNRs used for measurements of ISM composition (top part), and with detected ejecta (bottom part).

\begin{tabular}{|c|c|c|c|c|c|c|c|}
\hline \multirow{2}{*}{ MCSNR } & \multicolumn{4}{|c|}{ High $\mathrm{X} / \mathrm{Fe}$ flags } & \multicolumn{3}{|c|}{ Low X/Fe flags } \\
\hline & $\mathrm{O}$ & $\mathrm{Ne}$ & $\mathrm{Mg}$ & $\mathrm{Si}$ & $\mathrm{O}$ & $\mathrm{Ne}$ & $\mathrm{Mg}$ \\
\hline J0046-7308 & $\mathrm{Y}$ & $\mathrm{Y}$ & - & $\mathrm{Y}$ & - & - & - \\
\hline J0047-7308 & - & $\mathrm{Y}$ & $\mathrm{Y}$ & - & - & - & - \\
\hline J0047-7309 & $\mathrm{Y}$ & $\mathrm{Y}$ & $\mathrm{Y}$ & - & - & - & - \\
\hline J0049-7314 & - & - & - & - & $\mathrm{Y}$ & $\mathrm{Y}$ & $\mathrm{Y}$ \\
\hline J0051-7321 & $\mathrm{Y}$ & $\mathrm{Y}$ & $\mathrm{Y}$ & $\mathrm{Y}$ & - & - & - \\
\hline J0059-7210 & $\mathrm{Y}$ & $\mathrm{Y}$ & - & - & - & - & - \\
\hline J0103-7209 & - & $\mathrm{Y}$ & - & - & - & - & - \\
\hline J0104-7201 & $\mathrm{Y}$ & $\mathrm{Y}$ & $\mathrm{Y}$ & $\mathrm{Y}$ & - & - & - \\
\hline $\mathrm{J} 0105-7223$ & $\mathrm{Y}$ & $\mathrm{Y}$ & $\mathrm{Y}$ & $\mathrm{Y}$ & - & - & - \\
\hline $\mathrm{J} 0105-7210$ & - & - & - & - & $\mathrm{Y}$ & $\mathrm{Y}$ & $\mathrm{Y}$ \\
\hline \multirow[t]{3}{*}{ J0106-7205 } & - & - & - & - & $\mathrm{Y}$ & Y & Y \\
\hline & \multicolumn{7}{|c|}{ ISM abundances } \\
\hline & \multicolumn{2}{|c|}{$\mathrm{O}$} & \multicolumn{2}{|c|}{$\mathrm{Ne}$} & \multicolumn{2}{|c|}{$\mathrm{Mg}$} & $\mathrm{Fe}$ \\
\hline J0047-7308 & \multicolumn{2}{|c|}{-} & \multicolumn{2}{|c|}{-} & \multicolumn{2}{|c|}{-} & $\mathrm{Y}$ \\
\hline J0047-7309 & \multicolumn{2}{|c|}{-} & \multicolumn{2}{|c|}{-} & \multicolumn{2}{|c|}{-} & $\mathrm{Y}$ \\
\hline J0051-7321 & \multicolumn{2}{|c|}{$\mathrm{Y}$} & \multicolumn{2}{|c|}{$\mathrm{Y}$} & \multicolumn{2}{|c|}{$\mathrm{Y}$} & Y \\
\hline $\mathrm{J} 0052-7236$ & \multicolumn{2}{|c|}{$\mathrm{Y}$} & \multicolumn{2}{|c|}{$\mathrm{Y}$} & \multicolumn{2}{|c|}{$\mathrm{Y}$} & $\mathrm{Y}$ \\
\hline J0056-7209 & \multicolumn{2}{|c|}{$\mathrm{Y}$} & \multicolumn{2}{|c|}{$\mathrm{Y}$} & \multicolumn{2}{|c|}{$\mathrm{Y}$} & $\mathrm{Y}$ \\
\hline J0058-7217 & \multicolumn{2}{|c|}{$\mathrm{Y}$} & \multicolumn{2}{|c|}{ Y } & \multicolumn{2}{|c|}{$\mathrm{Y}$} & Y \\
\hline J0059-7210 & \multicolumn{2}{|c|}{-} & \multicolumn{2}{|c|}{-} & \multicolumn{2}{|c|}{$Y$} & Y \\
\hline $\mathrm{J} 0105-7223$ & \multicolumn{2}{|c|}{$\mathrm{Y}$} & \multicolumn{2}{|c|}{ Y } & \multicolumn{2}{|c|}{$\mathrm{Y}$} & $\mathrm{Y}$ \\
\hline
\end{tabular}

$6583 \AA$, $\mathrm{H} \alpha$ and $[\mathrm{S} \mathrm{II}]$ at 6717 and $6731 \AA$. The latter are shocksensitive line: the ratio of [S II] lines to $\mathrm{H} \alpha$ should be $\gtrsim 0.40$ for an object to be classified as an SNR shock from the point of view of optical spectra. Our MCSNR candidates have values of 0.47 and 0.46 (see also Table 2) so we can classify them as SNRs. Also, the ratio between the individual [S II] lines $(6717 \AA$ / $6731 \AA$ ) of 1.32 and 1.56 is fully in line with optical SNR spectra. The only exception are [N II] lines whose ratios to $\mathrm{H} \alpha$ have values of 0.39 and 0.42 , which is somewhat low. In Milky Way SNRs, this (mostly) would not be accepted as an SNR, however in the SMC it is different. From the early optical spectral observations of the MCs (see examples in Dopita 1979) it is well known that $\left[\mathrm{N}_{\mathrm{II}}\right]$ lines are very weak due to an abundance effect: nitrogen, particularly in the SMC, is even more underabundant (by 0.3-0.5 dex) than other elements (Russell \& Dopita 1992; Dopita et al. 2019). So, if we exclude the value and comparison of nitrogen lines with $\mathrm{H} \alpha$, all other spectral characteristics of these two candidates match SNRs.

\subsubsection{Notes on individual SNR candidates}

MCSNR candidate J0056-7209. It is a large optical shell of $99 \mathrm{pc}$ by $65 \mathrm{pc}$ in size (Fig. 1, left), among the largest SNRs in the SMC. The weak diffuse X-ray emission, first identified in Haberl et al. (2012b), is however confined to the northern region of the optical loop only, centred at $\mathrm{RA}(\mathrm{J} 2000)=00^{\mathrm{h}} 56^{\mathrm{m}} 33.0^{\mathrm{s}}$ and $\operatorname{Dec}(\mathrm{J} 2000)=-72^{\circ} 08^{\prime} 00^{\prime \prime}$ and with extent of about $48 \mathrm{pc}$. Using WiFeS spectroscopic data, we found a strong indication of shock excitation with $[\mathrm{S} \mathrm{II}] / \mathrm{H} \alpha$ ratio of 0.46 (Fig. 3, left), lending further support to a true SNR nature for this source. We also found that the weak $[\mathrm{O} \mathrm{III}]$ emission is coinciding with the X-ray emission of MCSNR candidate J0056-7209, which we confirm as of thermal nature with low metal abundances (Fig. B.9, Table A.3). The lack of radio continuum detection is surprising but not unheard of (see Venn diagrams of Leonidaki et al. 2010, B17), although this issue arises more commonly for galaxies beyond the Local Group where sources are often not spatially resolved. Based on optica and X-ray features, we can nevertheless confidently confirm this source as a bona-fide SNRs, attributing it the identifier MCSNR J0056-7209.

MCSNR candidate J0057-7211 (aka NS66D). It was first suggested as an SNR candidate based on the XMM-Newton mosaic of the SMC (Haberl et al. 2012b). The faint and extended soft X-ray emission is very close to the listed position, which is based on our new ASKAP EMU (SMC Early Science Project) radio continuum images (Joseph et al. 2019). The radio emission forms a partial shell at the north, while the diffuse thermal X-ray emission completes a shell in the south and south-western quadrant (Fig. B.9; note a likely unrelated point source at the south-western edge of the SNR). The low absorption measured in X-rays towards this SNR (Table A.3) suggests a position on the near side of the SMC. Its radio detection is clear, although it is a low surface brightness $\mathrm{SNR}\left(\mathrm{S}_{1 \mathrm{GHz}}=0.031 \mathrm{Jy}\right)$. Its radio $\mathrm{SED}$ is quite steep $(\alpha=-0.75 \pm 0.04)$. There is no obvious optical emission associated to that source (Fig. B.9), but the combination of radio and X-ray evidence leads us to conclude as a true SNR nature for it, to which we assign the identifier MCSNR J0057-7211. However, it is an optically "quiet" SNR, similar to some Galactic SNRs as described by Stupar et al. (2008).

MCSNR candidate J0106-7242. This is a newly suggested SMC SNR candidate based solely on its radio-continuum detection and morphology. We discovered this candidate in our new ASKAP radio continuum images (Joseph et al. 2019). Although a low surface brightness SNR candidate $\left(S_{1 \mathrm{GHz}}=0.0236 \mathrm{Jy}\right)$, its radio SED is typical for an SNR $(\alpha=$ $-0.55 \pm 0.02$, Fig. B.9). We do not detect any optical emission from this object - similarly to SMC SNR [HFP2000] 334. Also, we found no significant $\mathrm{X}$-ray detection, but this region is poorly covered with low exposure time ( $20 \mathrm{ks}$ only, combining all EPIC detectors). From the soft and medium fluxed mosaic image in and around the radio SNR position (Table A.2), we estimate a $3 \sigma$ upper limit of $1.9 \times 10^{34} \mathrm{erg} \mathrm{s}^{-1}$ for the $0.3-8 \mathrm{keV}$ luminosity (or $8.3 \times 10^{-15} \mathrm{erg} \mathrm{cm}^{-2} \mathrm{~s}^{-1} \operatorname{arcmin}^{-2}$ for surface brightness) of the MCSNR candidate J0106-7242. As several SMC SNRs are similarly faint (Table A.1), there is still comfortable room for a subsequent X-ray detection with deeper observations. Until then this object remains a good SNR candidate.

MCSNR candidate J0109-7318. We suggest this shelllike object (Fig. 1, right) as an SNR candidate because of its strong [S II] emission. Namely, its [S II]/H $\alpha$ ratio is 0.46 (Fig. 3, right), which indicates shock emission that could be attributed to an SNR nature. However, none of our present generation radio images show any signs of the object due to its projected proximity to a bright radio source (the background AGN XMMU J011053.5-731 415, Sturm et al. 2013a), whose sidelobes distort any nearby emission. The X-ray coverage is mediocre, and in addition it was covered at high off-axis angle in the two overlapping observations of that region, further decreasing the possibility of detecting associated emission. Like MCSNR candidate J0106-7242, this object remains a candidate awaiting $\mathrm{X}$-ray confirmation. 

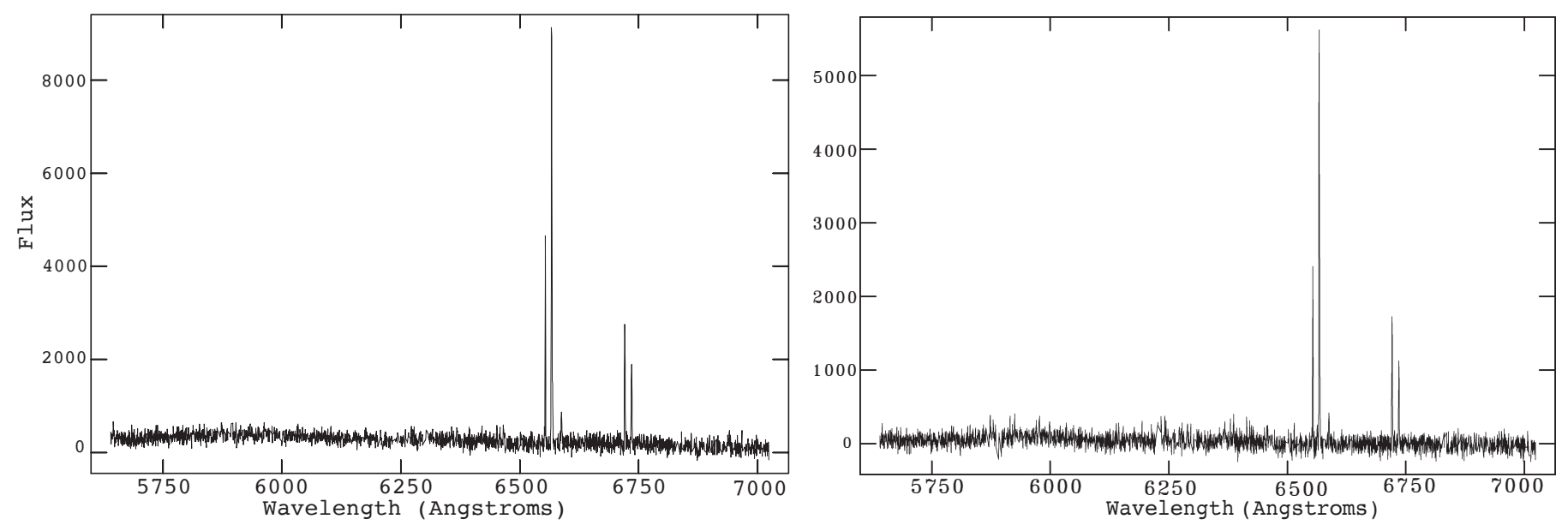

Fig. 3. Red part of optical spectra of SNR candidates J0056-7209 (left) and J0109-7318 (right) as seen by the WiFeS spectrograph. All main lines characteristics of old SNRs are seen: [N II] $\lambda \lambda 6548,6583 \AA$, H $\alpha$ and [S II] $\lambda \lambda 6717,6731 \AA$.

Table 2. Emission line intensities and ratios for two SNR candidates observed with the WiFeS spectrograph, taking $\mathrm{H} \alpha=100$.

\begin{tabular}{|c|c|c|c|c|c|c|c|c|c|c|c|c|}
\hline \multirow[t]{2}{*}{ Date } & \multirow{2}{*}{$\begin{array}{c}\text { Object name } \\
\text { MCSNR }\end{array}$} & \multicolumn{2}{|c|}{ Slit position (J2000) } & \multirow{2}{*}{$\begin{array}{r}{[\mathrm{N} \text { II }]} \\
6548 \AA\end{array}$} & \multirow[t]{2}{*}{$\mathrm{H} \alpha$} & \multirow{2}{*}{$\begin{array}{r}{[\mathrm{N} \text { II }]} \\
6583 \AA \\
\end{array}$} & \multirow{2}{*}{$\begin{array}{r}{[S \text { II }]} \\
6717 \AA \\
\end{array}$} & \multirow{2}{*}{$\begin{array}{r}{[S \mathrm{II}]} \\
6731 \AA \\
\end{array}$} & \multirow[t]{2}{*}[\mathrm{N}\mathrm{II}]{$/ \mathrm{H} \alpha$} & \multirow[t]{2}{*}[\mathrm{S}\text{II}]{$/ \mathrm{H} \alpha$} & \multirow{2}{*}{$\begin{array}{c}{[\text { S II] }} \\
6717 / 6731 \AA\end{array}$} & \multirow{2}{*}{$\begin{array}{c}\text { Electron density } \\
\left(\mathrm{cm}^{-3}\right)\end{array}$} \\
\hline & & RA & Dec & & & & & & & & & \\
\hline & J0056 & 0055 & -1 & 26. & $100^{(a)}$ & 14. & 27 & 20.4 & 0.42 & 0 . & 1. & $\sim 10^{2}$ \\
\hline $08 / 11 / 2015$ & J0109-7318 & 010947 & -731927 & 29.5 & $100^{(b)}$ & 9.1 & 28.1 & 18 & 0.39 & 0.46 & 1.56 & LDL \\
\hline
\end{tabular}

Notes. The rms wavelength dispersion error from the arc calibrations was $0.09 \AA$ while the relative percentage error in the flux determination from the calibration using the brightest lines was estimated as $\sim 13 \% .{ }^{(a)} \mathrm{H} \alpha$ flux $=19154$ counts; ${ }^{(b)} \mathrm{H} \alpha$ flux $=8837$ counts.

\subsection{Notes on individual SMC SNRs}

\subsubsection{MCSNR J0041-7336 (DEM S5)}

This SNR is a particularly large optical shell around a central X-ray emission (Fig. B.1), first studied with ROSAT and XMM-Newton in Haberl et al. (2000) and Filipović et al. (2008), respectively. The presence of an X-ray point-like source within the remnant associated to resolved radio emission recently led Alsaberi et al. (2019) to the discovery of a candidate pulsar wind nebula (PWN). The X-ray analysis of the diffuse emission (SNR component) in this work, which was also presented in Alsaberi et al. (2019), reveals that the emission arises from shocked ambient medium, as no abundance enhancement is found.

\subsubsection{The "triumvirate" of LHA 115-N 19}

In this large optical emission nebula lie the three SNRs MCSNR J0046-7308, J0047-7308, and J0047-7309. Although it is a confused region in the optical due to the bright emission and the lack of well-defined borders, these three sources have strongly different X-ray colours that allow us to distinguish them (Fig. B.2). The spectral fits indicate a similar temperature for these three SNRs $(\approx 0.6 \mathrm{keV})$. The variety in X-ray colours is instead due to variations in $N_{\mathrm{H}}$ (by up to an order of magnitude), ionisation age, and abundances. For instance, MCSNR J0047-7309 has highly elevated O, Ne, and $\mathrm{Mg}$ abundances (several times solar), while the strongest abundance enhancement of $\mathrm{J0046-7308}$ is $\mathrm{Si}$. $\mathrm{Ne}$ and $\mathrm{Mg}$ are also higher than solar in J0047-7308, while its high absorption $\left(N_{\mathrm{H}}=1.4 \pm 0.2 \times 10^{22} \mathrm{~cm}^{-2}\right)$ prevents a meaningful measurement of its oxygen abundance. Finally, we note that the large variation of $N_{\mathrm{H}}$ between these three objects indicates that even though close in projected position, they might be at different distances and not associated to the same star-forming event/region, as implicitly assumed in Auchettl et al. (2019).

\subsubsection{MCSNR J0048-7319 (IKT 4)}

This faint SNR has an irregular X-ray emission, filling a welldefined optical shell (Fig. B.1) and peaking in the Fe L-shell band, which led to the suggestion ( $\mathrm{vdH} 04$ ) that this was a type Ia SNR, similar to those discovered later by Borkowski et al. (2006a); Bozzetto et al. (2014); Maggi et al. (2014). We have doubled the exposure time compared to the first XMM-Newton analysis of vdH04, enabling us to confirm enhanced iron abundances. However, the $\mathrm{Mg}$ abundance is also formally enhanced in our spectral fits, making a conclusion as to the type of progenitor indecisive for IKT 4.

\subsubsection{MCSNR J0049-7314 (IKT 5)}

We found the interior X-ray emission to be enriched in iron (Fig. B.2), which we fit with a supplementary Fe-only component. The first component possibly shows enhanced $\mathrm{Mg}$ abundance, but not as markedly as in IKT 4 . This iron-rich core inside an [S II] shell and radio dimness make it very similar to other evolved type Ia SNRs found in the LMC.

\subsubsection{MCSNR J0051-7321 (IKT 6)}

The third brightest among SMC SNRs in X-rays, IKT 6 has two components, with ejecta-dominated emission in the centre, surrounded by a soft X-ray shell of shocked SMC-abundance ISM (Fig. B.3). The abundance pattern of the ejecta (elevated 
$\mathrm{Ne}, \mathrm{Mg}$, and $\mathrm{Si}$ ) betray a core-collapse $\mathrm{SN}$ origin. The shell can be used to measure SMC ISM abundances (Sect. 4.6).

\subsubsection{MCSNR J0052-7236}

Only the south-west part of this structure was suggested as an SNR, before Haberl et al. (2012b) suggested a possible close connection with other X-ray knots further north, linked by very faint emission. This could either be two close SNRs or a single large one. The slightly brighter SW X-ray knots correlate with the strongest optical emission, while the $\mathrm{N}$ part exhibits small filaments in [S II], possibly part of the remnant (Fig. B.3). Our X-ray spectral analysis reveals that the N and SW knots have strikingly similar spectra $\left(N_{\mathrm{H}}, k T\right.$, abundances). Combined with the morphology, we propose that these knots indeed form a single, large SNR, actually the largest SNR of the SMC.

\subsubsection{MCSNR J0058-7217 (IKT 16)}

This SNR is atypical in the SMC because of the hard extended source near its centre, suggested as the first PWN of the SMC (Owen et al. 2011) and then confirmed with high-resolution Chandra observations (Maitra et al. 2015). To characterise the extended soft X-ray emission from the underlying SNR, we included the pulsar and PWN components obtained in the Chandra analysis with fixed parameters in our analysis of the integrated emission. The X-ray size is roughly circular (1.2' radius) and matches some fait optical filamentary structure (Fig. B.4), albeit over a confused larger nebula.

\subsubsection{MCSNR J0059-7210 (IKT 18)}

Just $10^{\prime}$ north-east of IKT 16, IKT 18 has an irregular centrefilled X-ray morphology (Fig. B.4). Its location in a large optical nebula (N66) makes an optical identification difficult, but analysis of radio to $\mathrm{H} \alpha$ emission ratio allowed Ye et al. (1991) to separate the larger H II region from the SNR emission which matches the detected X-ray SNR fairly well. The abundances measured in the X-ray spectrum are low, except for $\mathrm{O}$ and $\mathrm{Ne}$ that are slightly above the SMC average values. This possibly points to ejecta contamination from regions we cannot pinpoint with the available data and spatial resolution of XMM-Newton.

\subsubsection{MCSNR J0100-7133 (DEM S108)}

The northernmost SNR of the SMC sample (by half a degree) is detected in radio, optical, and X-rays (Fig. B.5). The low surface brightness of the latter does not allow for a definite conclusion regarding elemental abundances. The diffuse interior X-ray emission is well outlined by an optical shell with enhanced [S II] and strong [O III] emission typical of radiative shocks (Cox \& Daltabuit 1971), indicating an evolved SNR.

\subsubsection{MCSNR J0103-7209 (IKT 21)}

Analysis of this SNR is complicated by the bright point-source AX J0103-722 within it, first identified in Hughes \& Smith (1994), and confirmed as a Be/X-ray binary by Israel et al. (2000). A compact radio and optical shell $\left(\approx 90^{\prime \prime}\right.$ diameter $)$ is seen around the X-ray binary (Fig. B.5), suggested as an SNR by Mathewson et al. (1984). No X-rays were found in this region (Ye et al. 1995) until the XMM-Newton analysis of vdH04, who modelled the faint thermal X-ray emission simultaneously with that of the binary. Much like for MCSNR J0052-7236, the
XMM-Newton mosaic (Haberl et al. 2012b) revealed a much larger diffuse emission than the former compact nebula. We used that $270^{\prime \prime}$ diameter region for our X-ray spectral analysis. We could thus afford to excise the X-ray binary point-source contribution by excluding a circle of 50 " radius (i.e. $90 \%$ encircled energy fraction at $8^{\prime}$ off-axis angle). This removes about $15 \%$ of the total SNR area. The emission was best fit with an NEI model, with only neon having a higher abundance than SMC ISM, which we take as a marginal indication of a core-collapse origin (Sect. 4.3.3). Finally, we note that the best-fit $N_{\mathrm{H}}$ of the large thermal SNR is about half that towards the X-ray binary $\left(3.9 \times 10^{21} \mathrm{~cm}^{-2}\right.$; Israel et al. 2000). In addition, the binary is far from the centre of our larger SNR, making an SNR-binary physical association far less likely than e.g. in SXP 1062 (Haberl et al. 2012c).

\subsubsection{MCSNR J0103-7247 ([HFP2000] 334)}

Without any detected optical emission (Fig. B.6), this object, discovered via its faint radio and X-ray emission (Filipović et al. 2008), was thought to host a putative PWN because of a central radio and X-ray point-like source. Later resolved with Chandra (Crawford et al. 2014), it was however attributed to a background object. We included the spectral parameters of that source obtained with Chandra (Crawford et al. 2014) in our analysis of the integrated X-ray emission. The extent of the SNR was measured using both radio and X-ray contours.

\subsubsection{MCSNR J0103-7201}

This faint SNR is detected as an $\mathrm{H} \alpha$ circular shell (Fig. B.6) centred on the long-period X-ray pulsar SXP 1323 (Gvaramadze et al. 2019). Deep radio and co-added Chandra data reveal faint radio and X-ray emission of the shell, thus confirming its SNR status (Haberl et al., in prep.). Interestingly, this is the second case in the SMC of an SNR containing a Be X-ray binary after SXP 1062 (see below), both objects harbouring long-period pulsars (>1000 s). Also, a recent SMC radio pulsar survey by Titus et al. (2019) did not reveal any coincident radio pulsars in this area even though the observations were specifically targeted to this area.

\subsubsection{MCSNR J0104-7201 (IKT 22)}

Most commonly known as 1E 0102.2-7219, this is the brightest X-ray and radio SNR in the SMC (Fig. B.6). It has been and continues to be extensively studied. Not wanting to expand on the bulk of past detailed studies, our approach was merely to include it in our analysis for completeness, and use a multi-component spectral model to derive consistently its X-ray luminosity. We needed three NEI components with variable abundances to satisfactorily reproduce the integrated emission. For pn spectra, a redshift parameter was added to account for small gain variations (Plucinsky et al. 2017) that shift the line centroid between observations and would otherwise cause large residuals. We have independently verified the lack of detected Fe K emission at high energies (Sect. 4.3.2).

\subsubsection{MCSNR J0105-7223 (IKT 23)}

This is the second brightest X-ray SNR of the SMC, although the radio flux density is about the median value of the whole sample. It is also very similar to IKT 6, with a soft ISM-abundance shell enclosing an ejecta-enhanced hotter plasma. The abundances of 
$\mathrm{O}$ and $\mathrm{Ne}$ are clearly super-solar, and in particular are enhanced relative to iron (Table A.3), yielding a clear CC SN origin for this remnant. In the optical, the remnant is not seen in [S II] or $\mathrm{H} \alpha$. However, a faint thin shell of [O III] emission delineates clearly the remnant on the outer side of the soft X-ray shell (Fig. B.7). This is strongly suggestive of the radiative part of the outer blast wave, from regions where the plasma cooled down below $\mathrm{X}$-ray emitting temperatures. Furthermore, this transition is very recent, as [O III] is emitted before $\mathrm{H} \alpha$ in cooling order.

\subsubsection{MCSNR J0105-7210 (DEM S128)}

This SNR is slightly elongated and consists mainly of diffuse interior X-ray emission and a faint radio shell encasing it at the northern and southern ends (Fig. B.7). In the optical only faint emission in the north can be associated to this object. The interior X-ray emission exhibits a strong iron enhancement, which was already seen with XMM-Newton (vdH04) and Chandra (Roper et al. 2015), that we interpret as a strong indicator of a type Ia SN origin (Sect. 4.7).

\subsubsection{MCSNR J0106-7205 (IKT 25)}

Bright in X-rays and optical but relatively radio-dim (Fig. B.8), J0106-7205 has a debated type. The elevated iron abundances measured with XMM-Newton (vdH04) and Chandra (Lee et al. 2011 ) led to the suggestions that it was a type Ia SNR, like IKT 5 and DEM S128. It was argued in Lopez et al. (2014) that the SMC abundance pattern used in the spectral analysis of Lee et al. (2011) was erroneous. While true, this does not affect the conclusion that the $\mathrm{Ne} / \mathrm{Fe}$ ratio was clearly skewed towards iron. We found a similar result in our re-analysis of the XMM-Newton data. On the contrary, Lopez et al. (2014) or Takeuchi et al. (2016), using Suzaku data, measured a Ne/Fe $\gtrsim 1$ ratio, albeit without resolving an $\mathrm{Ne} \mathrm{X}$ line that would be a tell-tale sign of enhanced neon enhancement. Meanwhile, the argument of the disrupted morphology being against a type Ia origin (Lopez et al. 2014) remains weak, as other SNRs have been found with elongated iron-rich cores where the optical emission does not follow the diffuse X-ray emission (e.g. DEM L238, DEM L249, or MCSNR J0527-7104, Borkowski et al. 2006a; Kavanagh et al. 2016), as is the case in IKT 25.

\subsubsection{MCSNR J0127-7333 (SXP 1062)}

Located far off to the south-east, in the Wing of the SMC, this SNR is observed as an optical shell (Hénault-Brunet et al. 2012), and radio and X-ray shell (Haberl et al. 2012c, Fig. B.8). The central source is an associated Be/X-ray binary, harbouring a long-period pulsar (SXP 1062). In this work we analysed the diffuse X-ray shell, including more observations that were obtained subsequently for the monitoring of the central binary (Sturm et al. 2013b).

\subsection{Abundances of the SMC ISM}

The current elemental abundances of the SMC ISM have been measured using several methods: (i) spectrophotometric observations of photospheric abundances of B stars (Hunter et al. 2009) or O-type dwarfs (Bouret et al. 2013), as these are young, short-lived stars and thus still presenting ISM abundances at their surfaces. Complications arise from the modelling of nonlocal thermodynamical equilibrium effects (e.g. Takeda et al. 2010) and the amount of rotational mixing (Heap et al. 2006). (ii) Photoionisation modelling of $\mathrm{H}$ II nebular spectra (Kurt et al. 1999; Peimbert et al. 2000; Testor 2001; Relaño et al. 2002; Peña-Guerrero et al. 2012; Carlos Reyes et al. 2015) remains affected by uncertainties of available atomic data, escape fraction, and incident spectra. (iii) Spectral modelling of radiative shocks in dense ISM clouds, as found within some SNRs (Russell \& Dopita 1990; Dopita et al. 2019).

Finally, in cases where the X-ray emission of SNRs is solely comprised of, or dominated by swept-up ISM, we can use the fitted abundances as measurements of the chemical composition of the ISM gas phase. This was used previously for the LMC (Hughes et al. 1998; Maggi et al. 2016; Schenck et al. 2016). One advantage is that it constrains directly the set of elements most relevant to X-ray observations, those which have emission lines and absorption edges in the $0.3-10 \mathrm{keV}$ band. SMC SNRs have been used previously for that purpose by vdH04, but only three objects were used in their study. Here, we attempt to improve this result, taking advantage of the higher number of SNRs known and observed.

Three SNRs (MCSNR J0052-7237, J0058-7217, and J0059-7210) had their abundances already measured in the fitting procedure of Sect. 4.3. To increase that number, we re-analyse the sample using their previous best-fit model and thawing the abundances of $\mathrm{O}, \mathrm{Ne}, \mathrm{Mg}$, and Fe. The fit improvements are not statistically significant, since by construction we would have identified these cases in Sect. 4.3. If the true abundances in an SNR are very close to the starting values of Russell \& Dopita (1992), there will be no strong improvement of the $\chi^{2}$-statistic. Therefore, we look instead at the uncertainties, that is, how well the abundance of a given element is constrained. Often the abundances are severely unconstrained (i.e. $\mathrm{X} / \mathrm{Fe}$ between a small fraction and hundred times the solar value) and we easily discard these objects as unsuitable. We add three SNRs (MCSNR J0047-7308, J0047-7309, and J0056-7209) to the sample from which (some) abundances can be measured. Although the latter is first presented here as an SNR candidate, we provided strong evidence to its confirmation as an SNR (Sect. 4.5), and at any rate its thermal emission is probing the gas-phase abundance of the ISM and can be used for that purpose.

Finally, the two bright objects MCSNR J0051-7321 and J0105-7223 clearly show two morphological components (see images in B), a central region of shocked ejecta surrounded by a shell of shocked ISM (vdH04; Hendrick et al. 2005; Schenck et al. 2014; Park et al. 2003). For these sources we go beyond the spatially-integrated analysis of Sect. 3.2. Using X-ray contours in soft and hard bands, we define an "interior" region (ejecta-rich) and a "shell" region (ISM), which is the whole SNR minus the interior region and a small buffer zone. This minimises cross-talk between regions and thus contamination of the shell with shocked ejecta emission. The shell emission is well fit by a vpshock model with low abundances ( $\sim 10 \%$ solar $)$. We thus obtain eight SNRs in which ISM abundances of various elements can be measured (Table 1). We list the mean abundances and uncertainties in Table 3, using the simple arithmetic mean for Col. (1) and a mean weighted in inverse proportion to each SNR uncertainty in Col. (2).

Before comparing with previous studies, we investigate two potentially important sources of systematic errors: The effects on the derived SMC abundances of the chosen NEI models, and of the abundance table used. Firstly, we replace the vpshock model by a vnei (single ionisation timescale) or vsedov (used in vdH04) in the analysis of the six SNRs where abundances other than just Fe were measured (Table 1). The spectral model 
Table 3. Abundances of the SMC ISM.

\begin{tabular}{cccccccc}
\hline \hline & $\begin{array}{c}\text { SMC SNRs } \\
(1)\end{array}$ & $\begin{array}{c}\text { weighted SNRs } \\
(2)\end{array}$ & $\begin{array}{c}\text { RD92 (H II + SNRs) } \\
\text { vdH04 (SNRs) }\end{array}$ & $\begin{array}{c}\text { Radiative shocks } \\
(5)\end{array}$ & $\begin{array}{c}\text { B stars } \\
(6)\end{array}$ & $\begin{array}{c}\text { H II regions } \\
(7)\end{array}$ \\
\hline $\mathrm{O}$ & $7.80_{-0.10}^{+0.38}$ & $7.61_{-0.16}^{+0.59}$ & $8.03 \pm 0.10$ & $8.13_{-0.16}^{+0.11}$ & $8.02 \pm 0.06$ & $7.99 \pm 0.21$ & $7.99 \pm 0.04$ \\
$\mathrm{Ne}$ & $7.17_{-0.11}^{+0.39}$ & $7.02_{-0.15}^{+0.54}$ & $7.27 \pm 0.20$ & $7.50 \pm 0.15$ & $7.04 \pm 0.10$ & - & $7.22 \pm 0.04$ \\
$\mathrm{Mg}$ & $6.76_{-0.13}^{+0.41}$ & $6.76_{-0.13}^{+0.42}$ & $6.98 \pm 0.12$ & $7.07 \pm 0.13$ & $(6.72)^{(a)}$ & $6.72 \pm 0.18$ & - \\
$\mathrm{Fe}$ & $6.60_{-0.14}^{+0.32}$ & $6.35_{-0.26}^{+0.58}$ & $6.84 \pm 0.13$ & $6.75 \pm 0.18$ & $(6.77)^{(a)}$ & - & - \\
{$[\mathrm{Fe} / \mathrm{H}]$} & $-0.83_{-0.14}^{+0.32}$ & $-1.08_{-0.26}^{+0.58}$ & $-0.59 \pm 0.13$ & $-0.68 \pm 0.18$ & -0.66 & - \\
{$[\mathrm{O} / \mathrm{Fe}]$} & $-0.06_{-0.18}^{+0.49}$ & $-0.01_{-0.60}^{+0.64}$ & $-0.07 \pm 0.16$ & $0.12_{-0.24}^{+0.21}$ & -0.01 & - \\
{$[\alpha / \mathrm{Fe}]$} & $-0.03_{-0.24}^{+0.75}$ & $0.06_{-0.63}^{+0.94}$ & $-0.06 \pm 0.15$ & $0.15_{-0.31}^{+0.29}$ & -0.04 & - & - \\
\hline
\end{tabular}

Notes. Elemental abundances are given as $12+\log (X / H)$; abundance ratios follow the convention $[X / Y]=\log (X / Y)-\log (X / Y)_{\odot}$. Columns $(1)-(4)$ are listing gas-phase abundance values, while Cols. (5)-(7) are total gas+dust abundances. ${ }^{(a)}$ Values assumed in their model.

References. References for (1) and (2) this work; (3) Russell \& Dopita (1992); (4) vdH04; (5) Dopita et al. (2019); (6) Hunter et al. (2009); (7) Testor (2001).
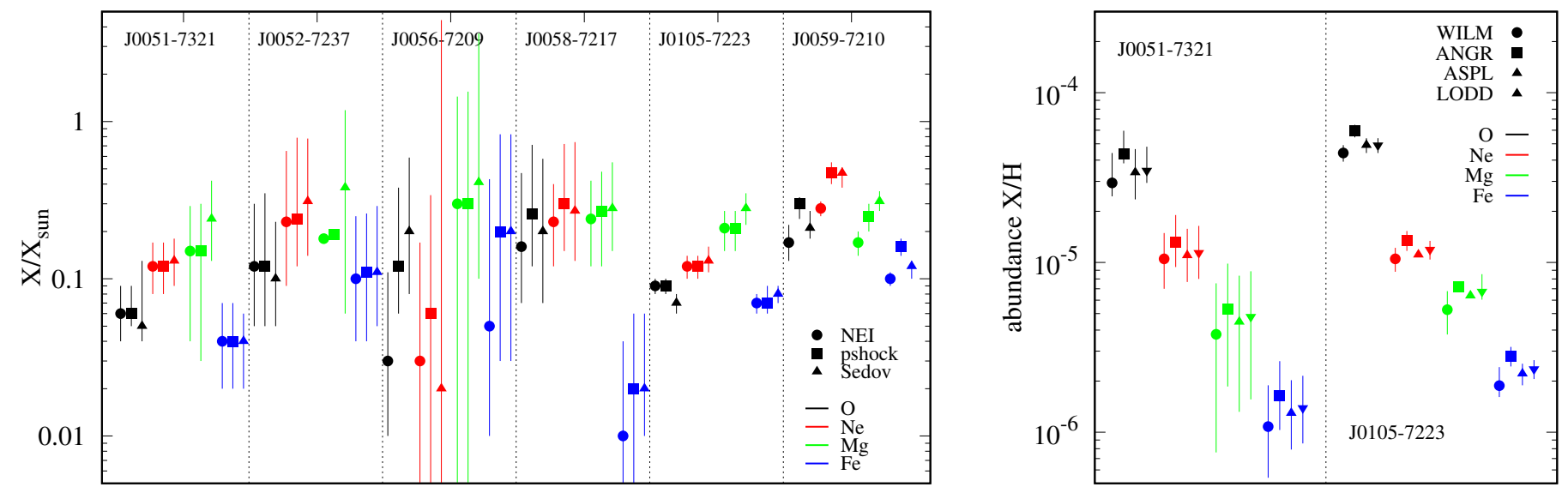

Fig. 4. Comparison of the effect of type of spectral models (left) and input abundance tables (right) on abundance derived with X-ray spectra of SNRs. Left: O, Ne, Mg, and Fe abundances (by group of three, from left to right, respectively) relative to the reference value of Wilms et al. (2000), in five SMC SNRs (labelled on top). Different symbols are used for the three types of spectral models used. Right: for two SNRs, derived number abundances relative to hydrogen as function of input abundance tables (reference in Sect. 4.6), each coded by different symbols.

chosen has no strong impact on the abundances, as shown on Fig. 4 (left). If anything, there is a tendency for the vnei model to yield slightly lower abundances. Results of a Sedov model, as used in vdH04 (SMC) and in Hughes et al. (1998) to measure LMC abundances, are essentially indistinguishable from those of the vpshock model.

Secondly, we used other abundance tables available in XSPEC (ANGR: Anders \& Grevesse 1989, LODD: Lodders 2003, ASPL: Asplund et al. 2009) to fit the spectra of MCSNR J0051-7321 and J0105-7223. The fitted abundances should be insensitive to the different starting points of these tables. However, they also differ in the abundances of trace elements (e.g. odd-Z nuclei) or other non-fitted elements, such as $\mathrm{Si}$ and $\mathrm{S}$. This affects the free electron balance $\left(n_{\mathrm{e}} / n_{\mathrm{H}}\right)$ and thus the emission continuum. Furthermore, in some cases rare elements have lines in similar energy bands as the fitted elements, like $\mathrm{N}, \mathrm{Ar}$, and $\mathrm{Ca}$ in the $0.5-0.6 \mathrm{keV}$ band dominated by oxygen. We show the absolute abundances obtained in Fig. 4 (right). There are no discernible differences. The abundance ratios such as $\mathrm{O} / \mathrm{Fe}$, should be the least affected by the choice of abundance tables. Indeed, the scatter is very small, with less than 5\% scatter between the four input tables.
Our results are best compared to those of vdH04 since they come from the same environment. In absolute abundances we find values lower by about $0.3-0.5$ dex. This could be ascribed to our larger sample, including several more evolved SNRs: as pointed in vdH04, larger remnants tend to have lower abundances $^{5}$, as they swept up more ISM and thus further dilute the effect of potential SN ejecta contamination. On that topic, we note that our derived abundance ratios $[\mathrm{O} / \mathrm{Fe}]$ or $[\alpha / \mathrm{Fe}]$ are well consistent (within $0.1 \mathrm{dex}$ ) with other studies, indicating that we have efficiently vetoed contamination by the more frequent CC SNRs ejecta, as we have shown for the LMC ISM as well (Hughes et al. 1998, MHK16).

We remind that our abundances are those of the (hot) gasphase. Compared to stellar abundance measurements (Hunter et al. 2009; Bouret et al. 2013), or dust depletion-corrected measurements (Dopita et al. 2019, Table 3), we found similar $\mathrm{Ne}$ abundance but lower $\mathrm{O}, \mathrm{Mg}, \mathrm{Fe}$ abundances, on average by $\approx 0.1-0.3 \mathrm{dex}$, which reflects partial depletion of these elements onto dust. However, the depletion factors $D_{\mathrm{X}}=\log \left(N_{\mathrm{X}} / N_{\mathrm{H}}\right)-$ $\log \left(N_{\mathrm{X}} / N_{\mathrm{H}}\right)_{\text {stellar }}$ are less (closer to 0 ) than in other ISM phases

\footnotetext{
5 We found a similar trend in our larger sample.
} 
Table 4. "Hint-spec" attributed to SNRs as function of spectral results.

\begin{tabular}{cc}
\hline \hline Hint-spec & Criteria \\
\hline 1 & At least three "low X/Fe" flags AND no "high X/Fe" flag \\
1.5 & (Two "low X/Fe" flags OR low O/Fe) AND no "high X/Fe" flag \\
2 & One "low X/Fe" flag (except O/Fe) AND "high X/Fe' flag \\
2.5 & Low Si/Fe AND no "high X/Fe" flag \\
3 & ISM abundances, unfitted abundances \\
3.5 & High Si/Fe AND no "low X/Fe" flag \\
4 & One "high X/Fe" flag (except O/Fe) AND no "low X/Fe" flag \\
4.5 & (Two "high X/Fe" flags OR high O/Fe) AND no "low X/Fe" flag \\
5 & (At least three "high X/Fe" flags AND no "low X/Fe" flag) OR pulsar/PWN detected \\
\hline
\end{tabular}

(e.g. $D_{\mathrm{Fe}} \approx-1$ in the warm ionised medium of $\mathrm{H}$ II regions). This can be explained by the (partial) destruction of dust by the SNR shocks (Borkowski et al. 2006b; Williams et al. 2006; Koo et al. 2016). At least a fraction of these elements are released into the gas phase and are contributing to the observed X-ray emission. Although we do not attempt to quantify this further, the average SMC SNR depletion is less than measured directly in radiative shocks, for instance in LMC SNRs (Dopita et al. 2016, 2018), probably an effect of the faster shocks probed in X-rays, that increase the intensity of dust grain destruction (Slavin et al. 2015).

\subsection{The ratio of $C C$ to type la $S N e$ in the $S M C$}

Here, we aim to establish the type (CC or Ia) of all SMC SNRs to measure $N_{\mathrm{CC}} / N_{\text {Ia }}$, the ratio of CC to Ia SNe rates. We covered the various methods of SNR typing in MHK16. We mostly use our $\mathrm{X}$-ray spectral results (i.e. the measurement of nucleosynthesis products in the ejecta), or the detection of an associated (NS) or PWN. We then add secondary evidence based on the local stellar environment of SMC SNRs to tentatively type the rest of the sample, a method we explain in detail in MHK16.

In Table 1 we flagged the detection of ejecta in 11 SNRs. As in MHK16, we assign a number "hint-spec" ranging from 1 (strongly favouring a type Ia origin) to 5 (strongly favouring a CC SN origin) depending on the flags raised, as summarised in Table 4. This leads to a (relatively) secure typing for 13 SNRs, including MCSNR J0058-7217 and J0127-7333, which host a PWN and a BeXRB, respectively. The remaining six SNRs can only be tentatively typed using the local stellar environment, which we characterised by two metrics as described in the following paragraphs.

First, we construct a $V$ vs. $(B-V)$ colour-magnitude diagram (CMD) of all stars within a projected distance of $100 \mathrm{pc}\left(\sim 5.7^{\prime}\right)$ of each SNR, using the photometric catalogue of Zaritsky et al. (2002, hereafter MCPS). We add stellar evolutionary tracks from Lejeune \& Schaerer (2001) to identify the upper main sequence of stars in the SMC, using initial masses from 3 to $40 M_{\odot}$ and a metallicity $Z=0.004=0.1 Z_{\odot}$. A distance modulus of $\mu=$ 18.89 is assumed, and the average extinction for "hot" SMC stars is taken as $A_{V}=0.6$ (Zaritsky et al. 2002). We use the criteria $V<16.4$ and $B-V<0.03$ to select blue early-type stars. The CMDs are shown in Appendix B. We denote $N_{\mathrm{OB}}$ the number of massive stars $\left(\gtrsim 8 M_{\odot}\right)$ in the vicinity of the remnant identified this way.

Second, we plot for each SNR the star formation rate (SFR) of its surroundings as a function of lookback time (Appendix B), obtained from the reconstructed SMC star formation history (SFH) of Harris \& Zaritsky (2004). Since the SMC SFH is noisier than in the LMC (Harris \& Zaritsky 2009), we take the average SFH in a grid of $3 \times 3$ cells centred on each SNR. We then compute $r=N_{\mathrm{CC}} / N_{\mathrm{Ia}}$, the ratio of CC SNe to thermonuclear $\mathrm{SNe}$ expected from the observed distribution of stellar ages in the neighbourhood of the remnants, as:

$r=\frac{\Psi_{1} M_{1}}{\Psi_{2} M_{2}+\Psi_{3} M_{3}}$

where $\Psi_{i}$ is the delay-time distribution, the SN rate following a star formation event, as measured by Maoz \& Badenes (2010) in the MCs, in time intervals $i=1,2$, and 3 corresponding to $t<35 \mathrm{Myr}, 35 \mathrm{Myr}<t<330 \mathrm{Myr}$, and $330 \mathrm{Myr}<t<14 \mathrm{Gyr}$, respectively. This $r$ provides us with a measure of the relative size of the pool of possible progenitors of both SN types, taking into account their delay-time distributions.

As massive stars are rarely formed in isolation, high values of $N_{\mathrm{OB}}$ and the CC-to-Type Ia SN ratio $r$ in a region hosting an SNR would strongly suggest a CC SN origin, while low values favour type Ia. We showed it to be the case in the LMC, where SNRs with well-established types (i.e. based on other methods) have bimodal distributions of $N_{\mathrm{OB}}$ and $r$ (MHK16). In the SMC we have the additional difficulty that there is significant extent along the line of sight, such that $N_{\mathrm{OB}}$ and $r$ might not reflect the correct environment of an SNR. For instance, an SNR might be located in front or behind a star forming region, without its progenitor drawn from that stellar population. If $N_{\mathrm{OB}}$ and $r$ are low, however, it is still a solid indication that no recent star formation occurred along the line of sight, as there is not enough internal extinction to mask the bright young stars that would have been created. Therefore, we can be relatively confident for typing SNRs with low $N_{\mathrm{OB}}$ and $r$ as type Ia, while classifying the high $N_{\mathrm{OB}}-r$ SNRs as $\mathrm{CC}$ should be done with caution.

The average $N_{\mathrm{OB}}$ is $128 \pm 67$ for $18 \mathrm{SNRs}^{6}$. It is higher $(160 \pm 63)$ for the ten secure CC SNRs than for the (only) three likely type Ia $(92 \pm 22)$, confirming that this dagnostic has some discriminatory power, even in the SMC. The average $N_{\mathrm{OB}}$ for the remaining six SNRs of uncertain type is 86 , with a very large scatter. It includes the two lowest occurrences, 18 around MCSNR J0040-7336 and 32 around J0100-7132. MCSNR J0056-7209 has $N_{\mathrm{OB}}=76$, less than all likely CC SNRs. The three other uncertain SNRs have $N_{\mathrm{OB}}$ between 117

6 MCSNR J0127-7333 is too far east to be in the area covered by the MCPS and will not be included in this discussion. It is however classified as a secure CC SNR based on its associated Be/X-ray binary. 
Table 5. "Hint-SF" attributed to SNRs as function of $N_{\mathrm{OB}}$ and $r$.

\begin{tabular}{|c|c|c|c|}
\hline$N_{N_{\mathrm{OB}}} r$-value & $r<0.6$ & $0.6<r<1.5$ & $r>1.5$ \\
\hline$N_{\mathrm{OB}}<80$ & 1 & 1.5 & 2 \\
\hline $80 \leq N_{\mathrm{OB}} \leq 115$ & 2.5 & 3 & 3.5 \\
\hline$N_{\mathrm{OB}}>11 \overline{5}$ & 4 & 4.5 & 5 \\
\hline
\end{tabular}

and 142, higher than those of type Ia SNRs and consistent with many CC SNRs.

Even when averaging over several cells, the SMC SFH is noisy, especially at recent times, which are critical. The distribution of $r$-values of all regions (not just those hosting an SNR) is less bimodal than in the LMC, without a prominent high- $r$ peak. This is again due to the elongated shape of the SMC along the line of sight. Older episodes of star-formation permeate most of the Cloud (Harris \& Zaritsky 2004) and are seen in projection in all the cells, thus lowering $r$ and blurring its peak in regions with recent star formation. Consequently, we put more emphasis on $N_{\mathrm{OB}}$ than $r$. We choose three intervals for $N_{\mathrm{OB}}$, each split in three intervals depending on $r$, to assign a number "hintSF" (for star formation) to our SNR, following the criteria from Table 5. As with "hint-spec", values close to 1 favour type Ia, and those close to 5 favour a CC origin. We combine the two hints by taking their weighted mean, with a coefficient of two for the "hint-spec" which is deemed to be more critical, since it does not have the projection effect of the star-formation hint. We take a slightly more conservative approach than in the LMC, classifying sources as 'likely-Ia' when the final hint is $<2.5$, and "likely-CC" if it is $>3.5$. Objects between 2.5 and 3.5 (inclusive) remain undecided. There are two SNRs in that category (MCSNR J0048-7319 and J0100-7133).

Out of 17 typed SNRs, we estimate $14 \mathrm{CC}$ and three type Ia SNRs. If we knew the true fraction of core-collapse SNRs $p_{\mathrm{CC}}$, the observed number $N_{\mathrm{CC}}$ would follow a binomial distribution for a given $N_{\text {typed }}$. We calculate the Bayesian posterior distribution of $p_{\mathrm{CC}}$ for the observed $N_{\mathrm{CC}}$, assuming a flat prior on $p_{\mathrm{CC}}$ between 0 and 1 . We measure a $90 \%$ confidence interval on $p_{\text {CC }}$ of $0.62-0.92$. To compare with the LMC results of MHK16, which presented the results in the form of a simple number ratio $N_{\text {CC }} / N_{\text {Ia }}=1.35$ (1.11-1.46), we conducted a similar analysis out of the 54 typed LMC SNRs. We find there a smaller value of $p_{\mathrm{CC}}$ between 0.46 and 0.68 (90\% confidence interval).

Therefore, despite a small overlap, the fraction of CC SNRs is higher in the SMC than in the LMC, or conversely, there are relatively more type Ia SNRs in the LMC. We argued in MHK16 that the apparent excess of type Ia SNe in the LMC, as compared to direct $\mathrm{SN}$ search in the local Universe or $N_{\mathrm{CC}} / N_{\text {Ia }}$ measurements from intracluster medium abundances, was due to the specific recent and intermediate age SFH of the LMC. Several studies found enhanced star formation episodes at 1.5-2 Gyr ago and 250-500 Myr ago, based on both CMD fitting of field stars (at various limiting magnitudes, Harris \& Zaritsky 2009; Weisz et al. 2013; Rubele et al. 2012; Meschin et al. 2014) and star cluster formation history (e.g. Baumgardt et al. 2013), which mostly agree with field star formation at recent times (Maschberger \& Kroupa 2011). Combined with the type Ia delay-time distribution, peaking below 1 Gyr (Maoz \& Mannucci 2012), there is a large pool of possible progenitors for type Ia SNRs.

In the SMC, several studies point to a major SFR enhancement about 5 Gyr ago (Rubele et al. 2015; Weisz et al. 2013; Cignoni et al. 2012; Noël et al. 2009), possibly related to early
LMC - SMC interaction, with only some evidence for a secondary peak at 1.5 Gyr ago (Rubele et al. 2015; Cignoni et al. 2012). Harris \& Zaritsky (2004) found the most significant intermediate star formation episode 2-3 Gyr ago. In recent times, SFR peaked again 200-400 Myr ago, most notably on the LMC side with the formation of the SMC Wing by tidal interaction. The smaller SMC SFR 0.5-1.5 Gyr ago as compared to the LMC could explain the currently smaller number of type Ia SNRs.

An important caveat, however, is that while recent star formation is strong in the Bar and Wing regions which are well covered with $X M M-N e w t o n$, the outskirts (at galactocentric radius larger than $1.5^{\circ}$ ) are poorly known, and might host more type Ia SNRs owing to the ancient SFH of these regions (e.g. Rubele et al. 2015). The detection of low surface brightness SNRs in these areas might be possible in the near future with the eROSITA all-sky survey and subsequent pointed surveys (Merloni et al. 2012).

Our suggested classification of three SNRs as type Ia is mostly driven by their X-ray spectral features, most notably the large $\mathrm{Fe}$ abundance of ejecta origin, and are considered robust. In no case is an SNR classified as type Ia based on local (projected) star-formation alone. MCSNR J0041-7336 (DEM S5) is far off to the south-west of the main SMC Bar and thus has the lowest recent star formation of the whole sample, but a PWN candidate was recently identified in it, strongly suggesting a CC SN origin. This explains the discrepancy with Auchettl et al. (2019) who list only one type Ia candidate SNR, but whose CC SNR classification is based on the projected star-formation history alone. Given the significant extent/depth of the SMC, such an interpretation is not warranted. A similar study for the LMC population, however, would be much more significant because the recent star forming regions and other regions potentially hosting type Ia SNRs are better segregated thanks to the thinness of the LMC and a favourable viewing angle.

\subsection{Radio properties, size, and morphology of SMC SNRs}

As in B17 we estimate the distributions of the radio parameters for the sample of 19 confirmed SMC SNRs using kernel smoothing with a Gaussian kernel. The maximum likelihood method with "leave one out" cross-validation is applied (Duin 1976) in order to calculate the optimal smoothing kernel bandwidth $(h)$. Confidence bands are calculated from $10^{4}$ bootstrap (Efron \& Tibshirani 1994) resamples. The kernel bandwidth, optimal for the original data sample, is used to calculate the resulting distributions of the bootstrap resamples. All distributions are calculated at 100 equidistant points along the plotted interval (on the $x$-axis, Fig. 5). At each $x$-axis coordinate we calculate the median value and the confidence bands as the $95 \%$ confidence interval around the median value. The same procedure and re-sampled data is also used to estimate uncertainties of the distribution mean, mode and median (Fig. 5). The boundary correction for the smoothed distributions that cannot have negative values (diameter and ovality) is done using the reflection method (Silverman 1986). We note that in B17 a different method (smooth bootstrap resampling) was used to estimate the optimal kernel bandwidth, but the method in this work is less computationally intensive and better suited to apply data reflection. For the flux density distribution we used a log scale.

Figure 5 shows estimates of the distributions for average diameter, ovality, radio spectral index and $1 \mathrm{GHz}$ flux density. The diameter and radio spectral index distributions appear to be symmetric. The ovality shows significant asymmetry with 

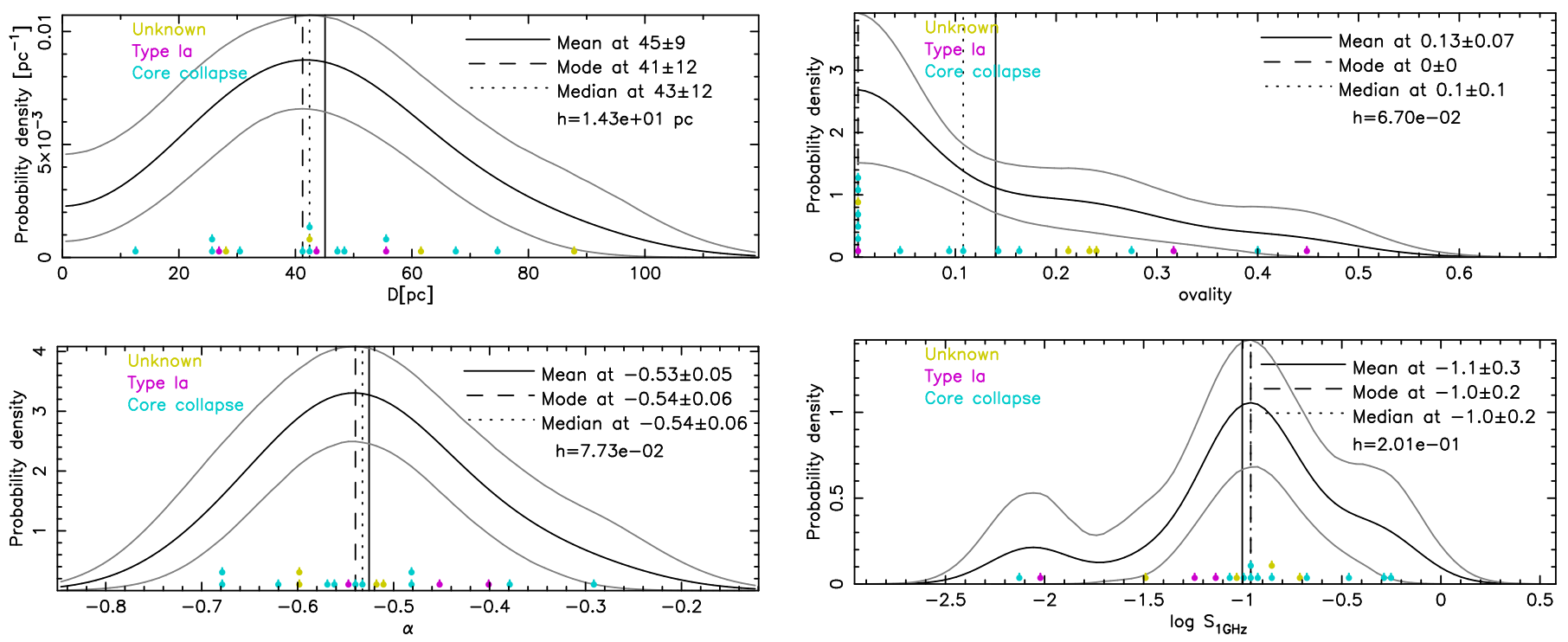

Fig. 5. Distribution of parameters of 19 confirmed SMC SNRs using kernel smoothing. The colour of symbols indicate progenitor type for a particular SNR. Data points that fall in the same bin on the $x$-axis are plotted within a vertical column with equidistant spacing. The upper and lower grey curves delineate the $95 \%$ uncertainty level. The corresponding kernel bandwidth $h$ and distribution parameters are shown on each panel. Top left: average diameter distribution. Top right: distribution of ovality, which is defined as $2\left(D_{\text {maj }}-D_{\min }\right) /\left(D_{\operatorname{maj}}+D_{\min }\right)$, where $D_{\text {maj }}$ and $D_{\text {min }}$ are the major and minor axes, respectively. Bottom left: radio spectral index distribution. Bottom right: estimate of the $1 \mathrm{GHz}$ flux density distribution for the 18 SNRs with available $1 \mathrm{GHz}$ flux density estimates from Table A.1.

many data points consistent with zero (circular morphology). The median ovality is the same within the uncertainties for SMC and LMC, although the SMC distribution contains a higher fraction of circular SNRs, pointing to a less disturbed ambient medium in that galaxy.

SNRs of the LMC population are slightly smaller (median of $33 \mathrm{pc}$ vs. $43 \mathrm{pc}$ in the $\mathrm{SMC}^{7}$ ). The observed difference might be due to the lower completeness of the LMC SNR population compared to that of the SMC because of the lower coverage fraction, for instance in X-rays (only central areas have been surveyed by XMM-Newton), if the outer area hosts on average larger, older SNRs, that could have been missed by previous surveys (for instance ROSAT all-sky survey and LMC pointed survey). Such incompleteness of the faint, large LMC SNR population was already suggested based on the X-ray luminosity function (MHK16). Another plausible factor for the smaller size of LMC remnants is an ambient medium denser on average than in the $\mathrm{SMC}$, which is expected given the concentration of gas and star formation in a disk in the LMC. This explanation is supported by the distribution of ambient densities shown in Fig. 6, where the density is estimated from the X-ray derived emission measure as $n \propto \sqrt{\mathrm{EM} / V}$ (see Sect. 4.3.1). The LMC exhibits a bimodal behaviour with about $25 \%$ of SNRs studied in X-rays interacting in a denser environment $\left(n>1 \mathrm{~cm}^{-3}\right)$ than the rest of the population, which clusters around $n \sim 0.1 \mathrm{~cm}^{-3}$. Only the latter, lower density mode $\left(n \lesssim 0.1 \mathrm{~cm}^{-3}\right)$ is seen for the SMC SNR population, the sole "high-density" SNR being J0104-7201 (IKT 22).

The distribution of spectral indices is the same in the SMC and LMC. This is likely due to the marginal dependency of $\alpha$ with age of the SNR (B17), and a similar contribution of PWNcontaminated SNRs, which if not properly resolved, would drive

\footnotetext{
7 In this work we used LMC values updated since B17, recalculating the distributions and their parameters with the same optimal kernel bandwidth and reflection methods used for the SMC.
}

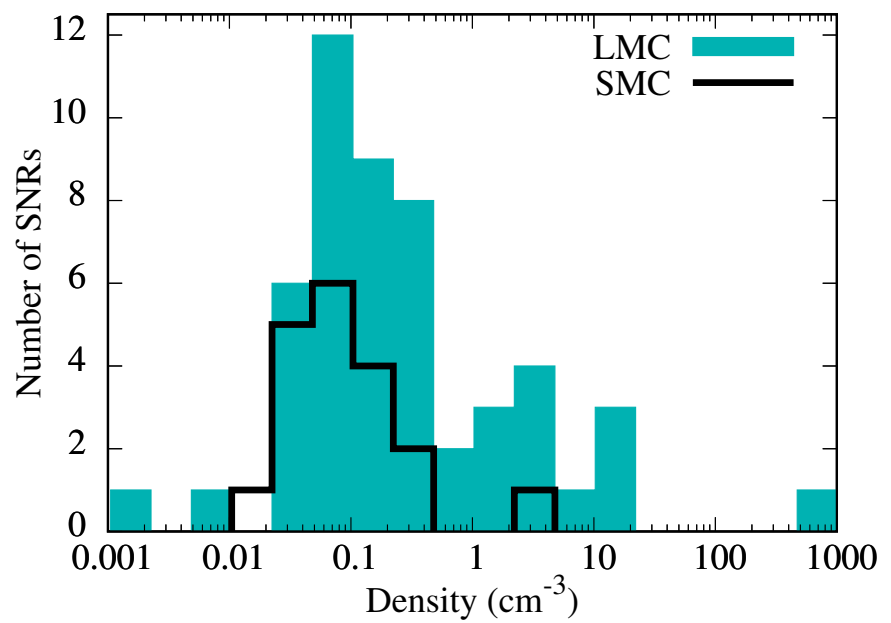

Fig. 6. Histogram of ambient density around LMC and SMC SNRs, estimated from the X-ray spectrum as $n \propto \sqrt{\mathrm{EM} / V}$ (see Sect. 4.3.1).

the radio spectra to flatter indices (e.g. Owen et al. 2011; Haberl et al. 2012a), in both galaxies.

Finally, the radio flux densities of SMC and LMC SNRs have consistent values, with the bulk of the population around 0.1 Jy. Chomiuk \& Wilcots (2009) already noted the similar radio luminosities across extragalactic SNR populations, which can be explained because the radio luminosity, that is, the synchrotron emission, is mostly controlled by the magnetic field strength. As SNR shocks amplify $B \propto \rho_{0} v_{\mathrm{s}}^{2}$ (with $\rho_{0}$ the ambient density and $v_{\mathrm{s}}$ the shock speed), the shock speeds and thus hydrodynamical states of the SNRs are more critical than the ambient density. Since most of the LMC and SMC are in the Sedov state, we can expect their radio luminosities to be similar.

We add on Fig. 7 the SMC objects to the $F_{\mathrm{X}}-S_{1 \mathrm{GHz}}$ diagram of B17. SMC SNRs appear to be fainter X-ray emitters than the LMC objects (median observed $0.3-8 \mathrm{keV}$ flux of 


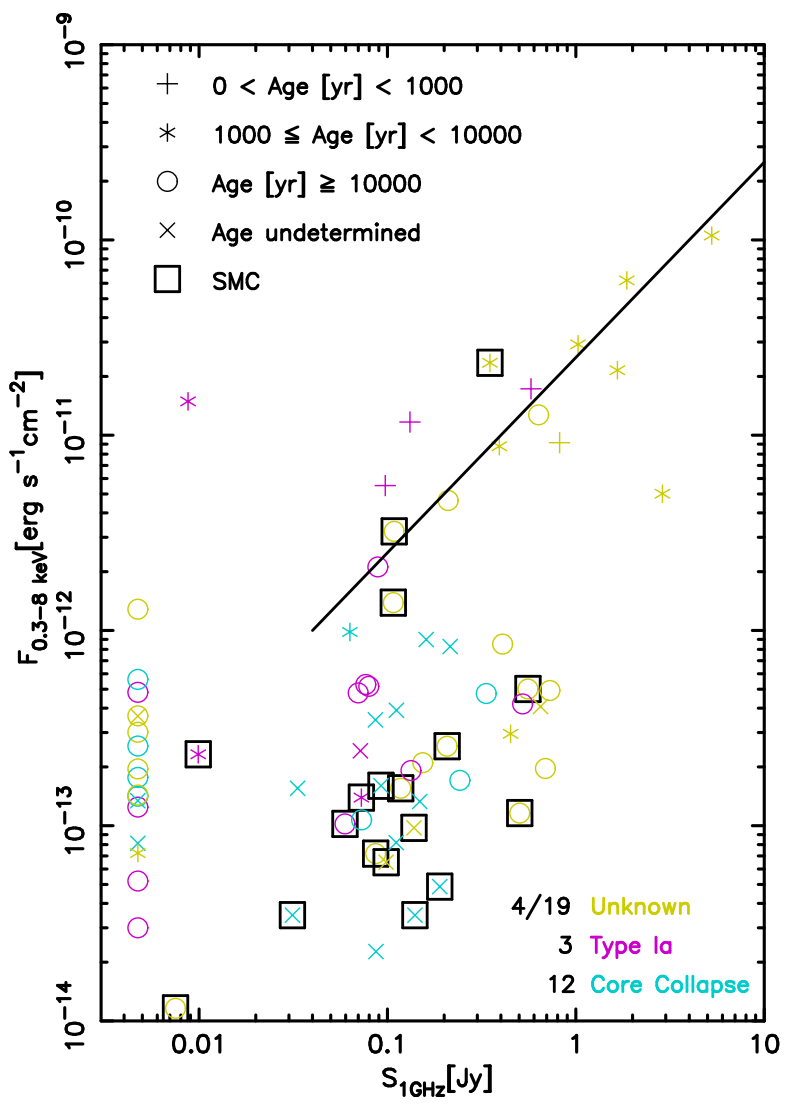

Fig. 7. Broad-band X-ray flux vs. $1 \mathrm{GHz}$ flux density for the sample of SMC and LMC SNRs with available data for age and explosion type (Table A.1, B17). The position of the colour-coded symbols along the axis with no measured radio flux is offset by -0.2 dex from the faintest detection. The solid line marks the high flux correlation from B17.

$1.2 \times 10^{-13}$ erg cm $\mathrm{cm}^{-2}$ vs. $4.1 \times 10^{-13} \mathrm{erg} \mathrm{cm}^{-2} \mathrm{~s}^{-1}$ ). This difference is likely again due to the lower average density in which SMC SNRs explode. The X-ray luminosity scales with the density squared and is thus much more affected by it than the radio luminosity (see above). The solid black line is plotted to guide the eye and indicates a linear correlation between X-ray and radio fluxes, which was noted for young $\left(\lesssim 10^{4} \mathrm{yr}\right)$ SNRs in B17. IKT 6 and IKT 23, two of the SMC SNRs close to that line, are however estimated to be older than $10^{4}$ yr (Hendrick et al. 2005; Schenck et al. 2014; Park et al. 2003), and are probably representing the extrapolation of that multi-wavelength correlation down to lower fluxes. This highlights the need for further, both theoretical and empirical, investigation in this direction to further examine the possible origin of this correlation.

\subsection{Three-dimensional spatial distribution}

$\mathrm{X}$-ray emitting objects in a galaxy are subjected to absorption by hydrogen and metals of that galaxy's ISM. These are obviously only sensitive to the amount of material between the source and the observer, while $21 \mathrm{~cm}$ observations can measure the total H I column density through a galaxy, in this case the SMC. Therefore, combining the equivalent $N_{\mathrm{H}}$ measured in X-rays $\left(=N_{\mathrm{H}}^{X}\right)$ with $N_{\mathrm{H}}^{21 \mathrm{~cm}}$, the line-of-sight integrated column density derived from H I surveys, gives us a proxy for the location of sources within the SMC along the line of sight.

As in MHK16, we define the " $N_{\mathrm{H}}$ fraction" as the ratio $N_{\mathrm{H}}^{X} / N_{\mathrm{H}}^{21 \mathrm{~cm}}$. It is a measurement of how deep an SNR is with respect to the H I structure. Such a relative line-of-sight proxy is particularly useful in the case of the SMC, because the main body of this galaxy has been shown to be inclined, with the north-eastern tip of the Bar closer than the south-western one by up to $10 \mathrm{kpc}$ (Subramanian \& Subramaniam 2012; Scowcroft et al. 2016).

The distribution of $N_{\mathrm{H}}$ fraction for 19 SMC SNRs observed in X-rays is shown in Fig. 8 (left) and compared to that of the LMC. The SMC distribution is flatter than in the LMC, where there is a strong mode at 0 and a second, fainter mode around 1 . This reflects the SMC neutral gas structure which has a large depth of 3-7.5 kpc (Subramanian \& Subramaniam 2009; North et al. 2010; Kapakos et al. 2011; Haschke et al. 2012), while that of the LMC has a well-defined thin disk distribution (Kim et al. 1999). Furthermore, there are no SMC SNRs with $N_{\mathrm{H}}$ fraction $>1$, while in the LMC this betrays the presence along the line of sight of foreground molecular clouds traced by $\mathrm{CO}$ emission (MHK16). Only MCSNR J0103-7201 (which we did not study with XMM-Newton) and the SNRs in the LHA 115N 19 complex (see Sect. 4.5.2) lie close in projection to some known giant molecular clouds (GMC) in the SMC (Mizuno et al. 2001; Leroy et al. 2007; Muller et al. 2010). In the latter case, MCSNR J0046-7308 is the best candidate to be physically associated with molecular clouds as evidenced by the detection of shocked CO emission in higher-resolution ALMA observations (Sano et al. 2019).

We find no obvious correlation of depth with spatial location (Fig. 8, right). SNRs clustered closely in projected position might be at widely different $N_{\mathrm{H}}$ fraction, and thus line-of-sight depth, in particular in the N19 region (south-west of SMC). This probably reflects the complex H I structure of that area, with two "sheets" of neutral gas (e.g. Stanimirović et al. 2004, Fig. 6).

\section{Summary}

We summarise below our work and findings:

- By combining deep, large scale XMM-Newton and radio surveys of the SMC, we presented a clean list of 19 bona-fide SNRs and identified 4 more candidates. Upon new optical spectroscopic observations and based on multiwavelength features, we confirm the two candidates MCSNR J0056-7209 and MCSNR J0057-7211 as bona-fide SNRs. we also argued against the SNR nature of six poorly studied objects which were previously classified as SNRs. This leads to a final list of 21 SNRs and 2 candidates in the SMC.

- We characterised the SNRs using a multiwavelength approach to best capture their size and morphology.

- The homogeneous X-ray spectral analysis allowed us to measure the hot-gas abundance of $\mathrm{O}, \mathrm{Ne}, \mathrm{Mg}$, and $\mathrm{Fe}$ to be between 0.1 and 0.2 times their solar values. $\mathrm{O}, \mathrm{Mg}$, and Fe are only partially depleted onto dust grains, as some of the grains have been destroyed by the fast shocks producing the X-ray emission through which we are measuring these abundances.

- We constrained $p_{\mathrm{CC}}$, the fraction of core-collapse $\mathrm{SN}$ for each SN in the SMC by using both intrinsic properties (detection of SN ejecta, presence of compact remnant) and extrinsic properties (local stellar population from which the $\mathrm{SN}$ progenitor is taken) to infer the type of each SNR. This fraction is between 0.62 and 0.92 ( $90 \%$ confidence interval), larger than that obtained from the same method in the LMC. This difference can potentially be attributed to an enhanced SFR episode 0.5-1.5 Gyr in the LMC which is not found in the SMC. Characterising the so far poorly-known SNR 

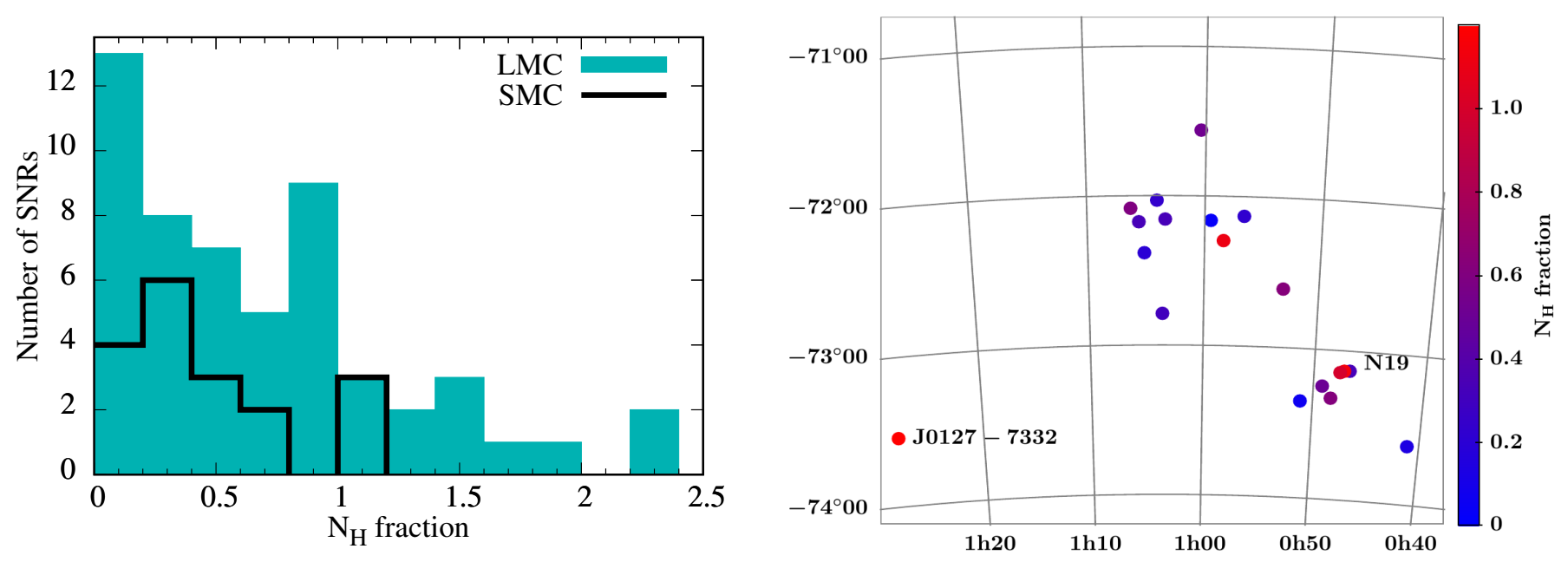

Fig. 8. Left: comparison of the distribution of $N_{\mathrm{H}}$ fraction for LMC and SMC SNRs. Right: spatial distribution of SMC SNRs, with $N_{\mathrm{H}}$ fraction used as a proxy for the line-of-sight depth within the neutral gas.

population on the outskirts of both Clouds, which is likely to preferentially contain type Ia SNRs, is needed to provide a more definitive answer.

- Radio properties like the spectral index and median flux density at $1 \mathrm{GHz}$ are remarkably consistent between the LMC and SMC population. This stems from the fact that such properties are governed by the ISM magnetic field and hydrodynamical states of the SNRs which are similar in both galaxies.

- LMC remnants are slightly smaller and more elongated than their SMC counterparts. A plausible explanation is a more disturbed and denser ambient medium in the LMC, as expected given the concentration of gas and star formation in the LMC disk or giant star forming complex (such as 30 Dor), where many SNRs explode.

- The SMC is inclined with respect to the plane of the sky and has significant depth, as opposed to the LMC. The line-ofsight proxy that is obtained by X-ray absorption reflects that fact. Although SNRs cannot be used as probes of absolute distances within the Cloud, we can show that several SNRs close in projection are likely to be at a different line-of-sight location. This should serve as an important caveat for studies that rely solely on the projected positions of these objects.

Acknowledgements. We thank the anonymous referee for their comments that helped to improve the presentation of our results. P. M. and J. B. acknowledge support by the Centre National d'Études Spatiales (CNES). BV acknowledges financial support from the Ministry of Education, Science and Technological Development of the Republic of Serbia through the project \#ON176005 "Emission nebulae: structure and evolution". M.S. acknowledges support by the Deutsche Forschungsgemeinschaft through the Heisenberg professor grants SA 2131/5-1 and 12-1. The Australian Compact Array and the Australian SKA Pathfinder (ASKAP) are part of the Australian Telescope which is funded by the Commonwealth of Australia for operation as National Facility managed by CSIRO. This paper includes archived data obtained through the Australia Telescope Online Archive (http://atoa.atnf.csiro.au). We used the KARMA and MIRIAD software packages developed by the ATNF. Operation of ASKAP is funded by the Australian Government with support from the National Collaborative Research Infrastructure Strategy. ASKAP uses the resources of the Pawsey Supercomputing Centre. Establishment of ASKAP, the Murchison Radio-astronomy Observatory and the Pawsey Supercomputing Centre are initiatives of the Australian Government, with support from the Government of Western Australia and the Science and Industry Endowment Fund. We acknowledge the Wajarri Yamatji people as the traditional owners of the Observatory site. This research has made use of Aladin, SIMBAD and VizieR, operated at the CDS, Strasbourg, France.

\section{References}

Alsaberi, R. Z. E., Maitra, C., Filipović, M. D., et al. 2019, MNRAS, 486, 2507

Alsabti, A. W., \& Murdin, P. 2017, Handbook of Supernovae (Berlin: Springer) Anders, E., \& Grevesse, N. 1989, Geochim. Cosmochim. Acta, 53, 197

Arnaud, K. A. 1996, in Astronomical Data Analysis Software and Systems V, eds. G. H. Jacoby, \& J. Barnes, ASP Conf. Ser., 101, 17

Asplund, M., Grevesse, N., Sauval, A. J., \& Scott, P. 2009, ARA\&A, 47, 481

Auchettl, K., Lopez, L. A., Badenes, C., et al. 2019, ApJ, 871, 64

Badenes, C., Maoz, D., \& Draine, B. T. 2010, MNRAS, 407, 1301

Balucinska-Church, M., \& McCammon, D. 1992, ApJ, 400, 699

Baumgardt, H., Parmentier, G., Anders, P., \& Grebel, E. K. 2013, MNRAS, 430 676

Bomans, D. J., Rossa, J., Weis, K., \& Dennerl, K. 2003, in A Massive Star Odyssey: From Main Sequence to Supernova, eds. K. van der Hucht, A. Herrero, \& C. Esteban, IAU Symp., 212, 637

Borkowski, K. J., Hendrick, S. P., \& Reynolds, S. P. 2006a, ApJ, 652, 1259

Borkowski, K. J., Williams, B. J., Reynolds, S. P., et al. 2006b, ApJ, 642, L141

Bouret, J.-C., Lanz, T., Martins, F., et al. 2013, A\&A, 555, A1

Bozzetto, L. M., Kavanagh, P. J., Maggi, P., et al. 2014, MNRAS, 439, 1110

Bozzetto, L. M., Filipović, M. D., Vukotić, B., et al. 2017, ApJS, 230, 2

Bruhweiler, F. C., Klinglesmith, III, D. A., Gull, T. R., \& Sofia, S. 1987, ApJ, 317,152

Carlos Reyes, R. E., Reyes Navarro, F. A., Meléndez, J., Steiner, J., \& Elizalde, F. 2015, Rev. Mex. Astron. Astrofis., 51, 135

Chieffi, A., \& Limongi, M. 2017, ApJ, 836, 79

Chomiuk, L., \& Wilcots, E. M. 2009, ApJ, 703, 370

Cignoni, M., Cole, A. A., Tosi, M., et al. 2012, ApJ, 754, 130

Cox, D. P., \& Daltabuit, E. 1971, ApJ, 167, 113

Crawford, E. J., Filipovic, M. D., de Horta, A. Y., et al. 2011, Serb. Astron. J., 183,95

Crawford, E. J., Filipović, M. D., McEntaffer, R. L., et al. 2014, AJ, 148, 99

De Luca, A., \& Molendi, S. 2004, A\&A, 419, 837

Dickey, J. M., \& Lockman, F. J. 1990, ARA\&A, 28, 215

Dopita, M. A. 1979, Aust. J. Phys., 32, 123

Dopita, M., Hart, J., McGregor, P., et al. 2007, Ap\&SS, 310, 255

Dopita, M. A., Seitenzahl, I. R., Sutherland, R. S., et al. 2016, ApJ, 826, 150

Dopita, M. A., Vogt, F. P. A., Sutherland, R. S., et al. 2018, ApJS, 237, 10

Dopita, M. A., Seitenzahl, I. R., Sutherland, R. S., et al. 2019, AJ, 157, 50

Duin, R. P. W. 1976, IEEE Trans. Comput., 25, 1175

Efron, B., \& Tibshirani, R. J. 1994, An Introduction to the Bootstrap (Boca Raton, US: CRC press)

Filipovic, M. D., Jones, P. A., White, G. L., et al. 1997, A\&AS, 121, 321

Filipovic, M. D., Haynes, R. F., White, G. L., \& Jones, P. A. 1998, A\&AS, 130, 421

Filipović, M. D., Bohlsen, T., Reid, W., et al. 2002, MNRAS, 335, 1085

Filipović, M. D., Payne, J. L., Reid, W., et al. 2005, MNRAS, 364, 217

Filipović, M. D., Haberl, F., Winkler, P. F., et al. 2008, A\&A, 485, 63

Findlay, J. W. 1966, ARA\&A, 4, 77

Finkelstein, S. L., Morse, J. A., Green, J. C., et al. 2006, ApJ, 641, 919 
For, B.-Q., Staveley-Smith, L., Hurley-Walker, N., et al. 2018, MNRAS, 480, 2743

Galvin, T. J., \& Filipovic, M. D. 2014, Serb. Astron. J., 189, 15

Galvin, T. J., Filipović, M. D., Tothill, N. F. H., et al. 2014, Ap\&SS, 353, 603

Garofali, K., Williams, B. F., Plucinsky, P. P., et al. 2017, MNRAS, 472, 308

Ghavamian, P., Raymond, J., Smith, R. C., \& Hartigan, P. 2001, ApJ, 547, 995

Graczyk, D., Pietrzyński, G., Thompson, I. B., et al. 2014, ApJ, 780, 59

Green, D. A. 2019, JApA, 40, 36

Gvaramadze, V. V., Kniazev, A. Y., \& Oskinova, L. M. 2019, MNRAS, 485, L6

Haberl, F., \& Pietsch, W. 2004, A\&A, 414, 667

Haberl, F., Filipović, M. D., Pietsch, W., \& Kahabka, P. 2000, A\&AS, 142, 41

Haberl, F., Filipović, M. D., Bozzetto, L. M., et al. 2012a, A\&A, 543, A154

Haberl, F., Sturm, R., Ballet, J., et al. 2012b, A\&A, 545, A128

Haberl, F., Sturm, R., Filipović, M. D., Pietsch, W., \& Crawford, E. J. 2012c, A\&A, 537, L1

Harris, J., \& Zaritsky, D. 2004, AJ, 127, 1531

Harris, J., \& Zaritsky, D. 2009, AJ, 138, 1243

Haschke, R., Grebel, E. K., \& Duffau, S. 2012, AJ, 144, 107

Heap, S. R., Lanz, T., \& Hubeny, I. 2006, ApJ, 638, 409

Hénault-Brunet, V., Oskinova, L. M., Guerrero, M. A., et al. 2012, MNRAS, 420, L13

Hendrick, S. P., Reynolds, S. P., \& Borkowski, K. J. 2005, ApJ, 622, L117

Hilditch, R. W., Howarth, I. D., \& Harries, T. J. 2005, MNRAS, 357, 304

Hughes, J. P., \& Smith, R. C. 1994, AJ, 107, 1363

Hughes, J. P., Hayashi, I., \& Koyama, K. 1998, ApJ, 505, 732

Hunter, I., Brott, I., Langer, N., et al. 2009, A\&A, 496, 841

Inoue, H., Koyama, K., \& Tanaka, Y. 1983, in Supernova Remnants and their X-ray Emission, eds. J. Danziger, \& P. Gorenstein, IAU Symp., 101, 535

Israel, G. L., Campana, S., Covino, S., et al. 2000, ApJ, 531, L131

Joseph, T. D., Filipović, M. D., Crawford, E. J., et al. 2019, MNRAS, 490, 1202

Kahabka, P., Pietsch, W., Filipović, M. D., \& Haberl, F. 1999, A\&AS, 136, 81

Kapakos, E., Hatzidimitriou, D., \& Soszyński, I. 2011, MNRAS, 415, 1366

Kavanagh, P. J., Sasaki, M., Bozzetto, L. M., et al. 2016, A\&A, 586, A4

Kim, S., Dopita, M. A., Staveley-Smith, L., \& Bessell, M. S. 1999, AJ, 118, 2797

Koo, B.-C., Lee, J.-J., Jeong, I.-G., Seok, J. Y., \& Kim, H.-J. 2016, ApJ, 821, 20

Koyama, K., Petre, R., Gotthelf, E. V., et al. 1995, Nature, 378, 255

Kuntz, K. D., \& Snowden, S. L. 2008, A\&A, 478, 575

Kurt, C. M., Dufour, R. J., Garnett, D. R., et al. 1999, ApJ, 518, 246

Lee, J.-J., Park, S., Hughes, J. P., Slane, P. O., \& Burrows, D. N. 2011, ApJ, 731 L8

Lejeune, T., \& Schaerer, D. 2001, A\&A, 366, 538

Leonidaki, I., Zezas, A., \& Boumis, P. 2010, ApJ, 725, 842

Leroy, A., Bolatto, A., Stanimirovic, S., et al. 2007, ApJ, 658, 1027

Leung, S.-C., \& Nomoto, K. 2018, ApJ, 861, 143

Lodders, K. 2003, ApJ, 591, 1220

Long, K. S., Blair, W. P., Winkler, P. F., et al. 2010, ApJS, 187, 495

Long, K. S., Kuntz, K. D., Blair, W. P., et al. 2014, ApJS, 212, 21

Lopez, L. A. 2014, in Supernova Environmental Impacts, eds. A. Ray, \& R. A. McCray, IAU Symp., 296, 239

Lopez, L. A., Castro, D., Slane, P. O., Ramirez-Ruiz, E., \& Badenes, C. 2014, ApJ, 788, 5

Mac Low, M.-M., \& Klessen, R. S. 2004, Rev. Mod. Phys., 76, 125

Maggi, P., \& Acero, F. 2017, A\&A, 597, A65

Maggi, P., Haberl, F., Kavanagh, P. J., et al. 2014, A\&A, 561, A76

Maggi, P., Haberl, F., Kavanagh, P. J., et al. 2016, A\&A, 585, A162

Maitra, C., Ballet, J., Filipović, M. D., et al. 2015, A\&A, 584, A41

Maoz, D., \& Badenes, C. 2010, MNRAS, 407, 1314

Maoz, D., \& Mannucci, F. 2012, PASA, 29, 447

Maschberger, T., \& Kroupa, P. 2011, MNRAS, 411, 1495

Mathewson, D. S., Ford, V. L., Dopita, M. A., et al. 1984, ApJS, 55, 189

Merloni, A., Predehl, P., Becker, W., et al. 2012, ArXiv e-prints [arXiv:1209.3114]

Meschin, I., Gallart, C., Aparicio, A., et al. 2014, MNRAS, 438, 1067

Millar, W. C., White, G. L., Filipović, M. D., et al. 2011, Ap\&SS, 332, 221

Millar, W. C., White, G. L., \& Filipovic, M. D. 2012, Serb. Astron. J., 184, 19

Misanovic, Z., Pietsch, W., Haberl, F., et al. 2006, A\&A, 448, 1247

Mizuno, N., Rubio, M., Mizuno, A., et al. 2001, PASJ, 53, L45

Muller, E., Ott, J., Hughes, A., et al. 2010, ApJ, 712, 1248

Noël, N. E. D., Aparicio, A., Gallart, C., et al. 2009, ApJ, 705, 1260

Nomoto, K., Kobayashi, C., \& Tominaga, N. 2013, ARA\&A, 51, 457

North, P., Gauderon, R., Barblan, F., \& Royer, F. 2010, A\&A, 520, A74

O'Brien, A. N., Filipović, M. D., Crawford, E. J., et al. 2013, Ap\&SS, 347, 159

Owen, R. A., Filipović, M. D., Ballet, J., et al. 2011, A\&A, 530, A132

Pannuti, T. G., Schlegel, E. M., Filipović, M. D., et al. 2011, AJ, 142, 20
Pannuti, T. G., Swartz, D. A., Laine, S., et al. 2015, AJ, 150, 91 Park, S., Hughes, J. P., Burrows, D. N., et al. 2003, ApJ, 598, L95 Payne, J. L., Filipović, M. D., Pannuti, T. G., et al. 2004a, A\&A, 425, 443 Payne, J. L., Filipović, M. D., Reid, W., et al. 2004b, MNRAS, 355, 44 Payne, J. L., White, G. L., Filipović, M. D., \& Pannuti, T. G. 2007, MNRAS, 376,1793

Peña-Guerrero, M. A., Peimbert, A., Peimbert, M., \& Ruiz, M. T. 2012, ApJ, 746, 115

Peimbert, M., Peimbert, A., \& Ruiz, M. T. 2000, ApJ, 541, 688

Pellegrini, E. W., Oey, M. S., Winkler, P. F., et al. 2012, ApJ, 755, 40

Pietrzyński, G., Graczyk, D., Gallenne, A., et al. 2019, Nature, 567, 200

Pietsch, W., Misanovic, Z., Haberl, F., et al. 2004, A\&A, 426, 11

Pietsch, W., Freyberg, M., \& Haberl, F. 2005, A\&A, 434, 483

Plucinsky, P. P., Beardmore, A. P., Foster, A., et al. 2017, A\&A, 597, A35

Reid, W. A., Payne, J. L., Filipović, M. D., et al. 2006, MNRAS, 367, 1379

Relaño, M., Peimbert, M., \& Beckman, J. 2002, ApJ, 564, 704

Roper, Q., McEntaffer, R. L., DeRoo, C., et al. 2015, ApJ, 803, 106

Rubele, S., Kerber, L., Girardi, L., et al. 2012, A\&A, 537, A106

Rubele, S., Girardi, L., Kerber, L., et al. 2015, MNRAS, 449, 639

Russell, S. C., \& Dopita, M. A. 1990, ApJS, 74, 93

Russell, S. C., \& Dopita, M. A. 1992, ApJ, 384, 508

Sano, H., Matsumura, H., Yamane, Y., et al. 2019, ApJ, 881, 85

Sasaki, M., Pietsch, W., Haberl, F., et al. 2012, A\&A, 544, A144

Sault, R. J., Teuben, P. J., \& Wright, M. C. H. 1995, in Astronomical Data Analysis Software and Systems IV, eds. R. A. Shaw, H. E. Payne, \& J. J. E. Hayes, ASP Conf. Ser., 77, 433

Schenck, A., Park, S., Burrows, D. N., et al. 2014, ApJ, 791, 50

Schenck, A., Park, S., \& Post, S. 2016, AJ, 151, 161

Scowcroft, V., Freedman, W. L., Madore, B. F., et al. 2016, ApJ, 816, 49

Seward, F. D., \& Mitchell, M. 1981, ApJ, 243, 736

Silverman, B. 1986, Density Estimation for Statistics and Data Analysis (Boca Raton, Florida: Chapman and Hall/CRC)

Slavin, J. D., Dwek, E., \& Jones, A. P. 2015, ApJ, 803, 7

Stanimirovic, S., Staveley-Smith, L., Dickey, J. M., Sault, R. J., \& Snowden, S. L. 1999, MNRAS, 302, 417

Stanimirović, S., Staveley-Smith, L., \& Jones, P. A. 2004, ApJ, 604, 176

Stiele, H., Pietsch, W., Haberl, F., et al. 2011, A\&A, 534, A55

Stupar, M., Parker, Q. A., \& Filipović, M. D. 2008, MNRAS, 390, 1037

Sturm, R. K. N. 2012, PhD Thesis, Fakultät für Physik, Technische Universität München, Germany

Sturm, R., Drašković, D., Filipović, M. D., et al. 2013a, A\&A, 558, A101

Sturm, R., Haberl, F., Oskinova, L. M., et al. 2013b, A\&A, 556, A139

Subramanian, S., \& Subramaniam, A. 2009, A\&A, 496, 399

Subramanian, S., \& Subramaniam, A. 2012, ApJ, 744, 128

Takeda, Y., Kambe, E., Sadakane, K., \& Masada, S. 2010, PASJ, 62, 1239

Takeuchi, Y., Yamaguchi, H., \& Tamagawa, T. 2016, PASJ, 68, S9

Testor, G. 2001, A\&A, 372, 667

Thielemann, F. K., Eichler, M., Panov, I. V., \& Wehmeyer, B. 2017, Annu. Rev. Nucl. Part. Sci., 67, 253

Titus, N., Stappers, B. W., Morello, V., et al. 2019, MNRAS, 487, 4332

van der Heyden, K. J., Bleeker, J. A. M., \& Kaastra, J. S. 2004, A\&A, 421, 1031

Vink, J. 2012, A\&ARv, 20, 49

Wang, Q., \& Wu, X. 1992, ApJS, 78, 391

Weisz, D. R., Dolphin, A. E., Skillman, E. D., et al. 2013, MNRAS, 431, 364

Williams, B. J., Borkowski, K. J., Reynolds, S. P., et al. 2006, ApJ, 652, L33

Wilms, J., Allen, A., \& McCray, R. 2000, ApJ, 542, 914

Wong, G. F., Filipovic, M. D., Crawford, E. J., et al. 2011a, Serb. Astron. J., 182, 43

Wong, G. F., Filipovic, M. D., Crawford, E. J., et al. 2011b, Serb. Astron. J., 183,

Wong, G. F., Crawford, E. J., Filipovic, M. D., et al. 2012a, Serb. Astron. J., 184, 93

Wong, G. F., Filipovic, M. D., Crawford, E. J., et al. 2012b, Serb. Astron. J., 185, 53

Wongwathanarat, A., Janka, H. T., \& Müller, E. 2013, A\&A, 552, A126

Xi, L., Gaetz, T. J., Plucinsky, P. P., \& Hughes, J. P., \& Patnaude, D. J. 2019, ApJ, 874, 14

Yamaguchi, H., Sawada, M., \& Bamba, A. 2010, ApJ, 715, 412

Yamaguchi, H., Badenes, C., Petre, R., et al. 2014, ApJ, 785, L27

Ye, T., \& Turtle, A. J., \& Kennicutt, Jr. R. C. 1991, MNRAS, 249, 722

Ye, T. S., Amy, S. W., Wang, Q. D., Ball, L., \& Dickel, J. 1995, MNRAS, 275, 1218

Yew, M., Filipović, M. D., Roper, Q., et al. 2018, PASA, 35, e015

Yokogawa, J., Imanishi, K., Tsujimoto, M., et al. 2000, ApJS, 128, 491

Zaritsky, D., Harris, J., Thompson, I. B., \& Grebel, E. K., \& Massey, P. 2002, AJ, 123, 855 


\section{Appendix A: List and properties of SMC SNRs}

We list here the confirmed (Table A.1) and candidate (Table A.2) SNRs of the SMC, with their names, position and size, X-ray, optical, radio main properties, and hints regarding their type. In Table A.3 we give the spectral parameters of confirmed and candidate SNRs with existing XMM-Newton analysis. All relevant parameters are listed with their $90 \%$ C.L. uncertainties: the fitted SMC absorption column density (Col. 2), plasma temperature $k T$ (3), ionisation age $\tau$ (4), emission measure $\mathrm{EM}=n_{\mathrm{d}} n_{\mathrm{H}} V(5)$, and abundances (6). When a second component is used (in five SNRs), its parameters are given in Cols. (7)-(11). The first component is the one with higher EM. The quality of the fits are evaluated by the $\chi^{2} / v\left(=\chi_{\text {red }}^{2}\right)$ of Col. (12), where $v$ is the number of degrees of freedom. The median $\chi_{\text {red }}^{2}$ is $1.16 .90 \%$ of the fitted objects have a reduced $\chi^{2}$ less than 1.24. For the best-fit model, we derive the total observed (not corrected for abosrption) $0.3-8 \mathrm{keV} \mathrm{X}$-ray flux. We convert it to an X-ray luminosity assuming a common distance of $60 \mathrm{kpc}$ to all SNRs, which we list with other multiwavelength properties in the Table A.1.

Table A.1. SNRs in the SMC.

\begin{tabular}{|c|c|c|c|c|c|c|c|c|c|c|c|c|c|c|c|c|c|}
\hline Name & Other name & RA & Dec & $D_{\text {maj }} \times D_{\min }$ & $\mathrm{PA}$ & $D_{\mathrm{av}}$ & $L_{\mathrm{X}}$ & $N_{\mathrm{H}}$ fraction & $S_{1 \mathrm{GHz}}$ & $\alpha \pm \Delta \alpha$ & $\Sigma_{1 \mathrm{GHz}}\left(\times 10^{-20}\right)$ & {$[\mathrm{S} \mathrm{II}] / \mathrm{H} \alpha$} & $N_{\mathrm{OB}}$ & $r$ & & Hints & \\
\hline $\begin{array}{l}\text { MCSNR } \\
\text { (1) }\end{array}$ & (2) & $\begin{array}{c}(\mathrm{J} 2000) \\
(3)\end{array}$ & $\begin{array}{c}(\mathrm{J} 2000) \\
(4)\end{array}$ & $\begin{array}{l}\left({ }^{\prime \prime}\right) \\
(5)\end{array}$ & $\begin{array}{l}\left({ }^{\circ}\right) \\
(6)\end{array}$ & $\begin{array}{l}\text { (pc) } \\
(7)\end{array}$ & $\begin{array}{c}\left(10^{35} \mathrm{erg} \mathrm{s}^{-1}\right) \\
(8)\end{array}$ & (9) & $\begin{array}{l}\text { (Jy) } \\
(10)\end{array}$ & (11) & $\begin{array}{c}\left(\mathrm{W} \mathrm{m}{ }^{-2} \mathrm{~Hz}^{-1} \mathrm{sr}^{-1}\right) \\
\text { (12) }\end{array}$ & (13) & (14) & (15) & $\begin{array}{c}\text { (Spec. } \\
(16)\end{array}$ & $\mathrm{SF}$ & Final) \\
\hline J0041-7336 & DEM S5 & 00:41:01.7 & $-73: 36: 30$ & $245 \times 219$ & 105 & 67.6 & 0.42 & $0.14_{-0.14}^{+0.31}$ & 0.1383 & $-0.29 \pm 0.01$ & 0.1389 & 0.8 & 18 & $0.44_{-0.18}^{+2.55}$ & 5 & 1 & 3.67 \\
\hline J0046-7308 & [HFP2000] 414 & $00: 46: 40.6$ & $-73: 08: 15$ & $185 \times 140$ & 35 & 46.8 & 0.67 & $0.33_{-0.09}^{+0.15}$ & 0.1176 & $-0.38 \pm 0.05$ & 0.2460 & 0.4 & 135 & $0.94_{-0.56}^{+0.48}$ & 5 & 4.5 & 4.83 \\
\hline J0047-7308 & IKT2 & $00: 47: 16.6$ & $-73: 08: 36$ & $110 \times 100$ & 45 & 30.5 & 0.50 & $1.16_{-0.18}^{+0.18}$ & 0.5037 & $-0.54 \pm 0.04$ & 2.4740 & 0.6 & 139 & $0.94_{-0.56}^{+0.48}$ & 4.5 & 4.5 & 4.5 \\
\hline J0047-7309 & & $00: 47: 36.5$ & $-73: 09: 20$ & $180 \times 120$ & 75 & 42.8 & 1.10 & $1.00_{-0.20}^{+0.10}$ & 0.2074 & $-0.56 \pm 0.04$ & 0.5199 & 0.6 & 133 & $1.00_{-0.60}^{+0.35}$ & 5 & 4.5 & 4.83 \\
\hline J0048-7319 & IKT 4 & $00: 48: 19.6$ & $-73: 19: 40$ & $165 \times 130$ & 90 & 42.6 & 0.15 & $0.62_{-0.28}^{+0.35}$ & 0.1399 & $-0.60 \pm 0.04$ & 0.3531 & 0.4 & 142 & $0.85_{-0.50}^{+0.43}$ & 2.5 & 4.5 & 3.17 \\
\hline J0049-7314 & IKT 5 & 00:49:07.7 & $-73: 14: 45$ & $190 \times 190$ & 0 & 55.3 & 0.60 & $0.51_{-0.23}^{+0.25}$ & 0.0727 & $-0.45 \pm 0.04$ & 0.1091 & 0.7 & 104 & $0.87_{-0.51}^{+0.41}$ & 1 & 3 & 1.67 \\
\hline J0051-7321 & IKT 6 & 00:51:06.7 & $-73: 21: 26$ & $145 \times 145$ & 0 & 42.2 & 5.98 & $0.07_{-0.01}^{+0.02}$ & 0.1074 & $-0.57 \pm 0.04$ & 0.2767 & 0.5 & 128 & $0.83_{-0.48}^{+0.44}$ & 5 & 4.5 & 4.83 \\
\hline J0052-7236 & DEM S68 & 00:52:59.9 & $-72: 36: 47$ & $340 \times 270$ & 135 & 88.2 & 0.69 & $0.63_{-0.27}^{+0.57}$ & 0.0924 & $-0.52 \pm 0.02$ & 0.0545 & 0.4 & 130 & $1.60_{-1.16}^{+0.31}$ & 3 & 5 & 3.67 \\
\hline J0058-7217 & IKT 16 & $00: 58: 22.4$ & $-72: 17: 52$ & $256 \times 256$ & 0 & 74.5 & 0.31 & $1.12_{-0.33}^{+0.36}$ & 0.0866 & $-0.53 \pm 0.03$ & 0.0715 & 0.25 & 134 & $0.92_{-0.64}^{+0.92}$ & 5 & 4.5 & 4.83 \\
\hline J0059-7210 & IKT 18 & $00: 59: 27.7$ & $-72: 10: 10$ & $140 \times 140$ & 0 & 40.7 & 2.16 & $0.03_{-0.03}^{+0.02}$ & 0.5569 & $-0.48 \pm 0.03$ & 1.5389 & 1.7 & 204 & $1.37_{-0.95}^{+0.72}$ & 4.5 & 4.5 & 4.5 \\
\hline J0100-7133 & DEM S108 & 01:00:23.9 & $-71: 33: 41$ & $210 \times 210$ & 0 & 61.1 & 0.21 & $0.53_{-0.38}^{+0.85}$ & 0.1895 & $-0.51 \pm 0.02$ & 0.2327 & [O III] & 32 & $4.48_{-2.18}^{+3.21}$ & 3 & 2 & 2.67 \\
\hline J0103-7209 & IKT 21 & 01:03:17.0 & $-72: 09: 42$ & $270 \times 270$ & 0 & 78.5 & 0.28 & $0.33_{-0.16}^{+0.24}$ & 0.0984 & $-0.68 \pm 0.03$ & 0.6802 & 0.5 & 260 & $2.82_{-1.95}^{+0.09}$ & 4 & 5 & 4.33 \\
\hline J0103-7247 & [HFP2000] 334 & 01:03:29.1 & $-72: 47: 33$ & $105 \times 85$ & 0 & 27.5 & 0.15 & $0.31_{-0.19}^{+0.26}$ & 0.0313 & $-0.60 \pm 0.05$ & 0.1896 & - & 117 & $1.76_{-1.00}^{+0.51}$ & 3 & 5 & 3.67 \\
\hline J0103-7201 & & 01:03:36.5 & $-72: 01: 35$ & $98 \times 83$ & 90 & 26.2 & - & - & - & - & - & 0.5 & 296 & $2.29_{-1.56}^{+0.50}$ & 5 & 5 & 5 \\
\hline J0104-7201 & 1E 0102.2-7219 & 01:04:01.2 & $-72: 01: 52$ & $45 \times 43$ & 0 & 12.8 & 101.2 & $0.23_{-0.001}^{+0.001}$ & 0.3500 & $-0.68 \pm 0.02$ & 9.7926 & [O III] & 291 & $2.67_{-1.80}^{+0.36}$ & 5 & 5 & 5 \\
\hline J0105-7223 & IKT 23 & 01:05:04.2 & $-72: 23: 10$ & $192 \times 192$ & 0 & 55.9 & 13.86 & $0.20_{-0.03}^{+0.03}$ & 0.1086 & $-0.62 \pm 0.02$ & 0.1595 & {$[\mathrm{O} \mathrm{II}] ?$} & 96 & $2.82_{-1.87}^{+0.94}$ & 5 & 3.5 & 4.5 \\
\hline $\mathrm{J} 0105-7210$ & DEM S 128 & $01: 05: 30.5$ & $-72: 10: 40$ & $190 \times 120$ & 150 & 43.9 & 0.44 & $0.33_{-0.19}^{+0.27}$ & 0.0595 & $-0.55 \pm 0.03$ & 0.1413 & 0.6 & 110 & $2.77_{-1.86}^{+0.20}$ & 1 & 3.5 & 1.83 \\
\hline J0106-7205 & IKT 25 & $01: 06: 17.5$ & $-72: 05: 34$ & $110 \times 80$ & 25 & 27.3 & 1.00 & $0.60_{-0.07}^{+0.17}$ & 0.0099 & $-0.40 \pm 0.03$ & 0.0609 & 0.4 & 61 & $2.68_{-1.79}^{+0.24}$ & 1 & 2 & 1.33 \\
\hline J0127-7333 & SXP1062 & $01: 27: 44.1$ & $-73: 33: 01$ & $166 \times 166$ & 0 & 48.3 & 0.05 & $3.40_{-0.70}^{+0.63}$ & 0.0075 & $-0.48 \pm 0.05$ & 0.0148 & [O III] & - & - & 5 & - & 5 \\
\hline
\end{tabular}

Notes. The columns are the following: (1) MCSNR identifier, in the form JHHMM-DDMM. (2) Old "common" name used in the literature. (3) and (4) Position of the remnant, in J2000 equinox. (5) SNR extent using radio, optical, and X-ray emission (see Sect. 4.1). Major and minor axes are given in arcsecond. The error in diameter is usually smaller than $2^{\prime \prime}$. (6) Position angle of the SNR, in degrees, increasing in the north-east-south direction. A 0 value is given in circular cases. (7) Average size in parsec, assuming a common distance to all sources of $60 \mathrm{kpc}$. (8) $L_{\mathrm{X}}$, the X-ray luminosity in the $0.3-8 \mathrm{keV}$ band, in units of $10^{35} \mathrm{erg} \mathrm{s}^{-1}$, obtained as described in Sect. 4.3.1, at $60 \mathrm{kpc}$. (9) $N_{\mathrm{H}}$ fraction, as defined in Sect. 4.9. Uncertainties are given at the $90 \%$ C.L. (10) $1 \mathrm{GHz}$ flux density in Jansky. Errors are typically less than 10\%. (11) Radio spectral index with corresponding uncertainties. (12) $1 \mathrm{GHz}$ surface brightness. (13) Optical [S II]/H $\alpha$ ratio measured in MCELS images. [O III] indicates that the given SNR is dominated by [O III] $\lambda 5007 \AA$ emission. (14) $N_{\mathrm{OB}}$, the number of blue early-type stars within $100 \mathrm{pc}$ of the remnant, and (15) $r$, the ratio of CC SNe to thermonuclear SNe expected from the observed distribution of stellar ages in the neighbourhood of the remnant, as obtained by Eq. 3 (see Sect. 4.7). (16) "Hints" to the type of SN progenitor, based on spectral properties, local star formation, and a combination of both, as described in Sect. 4.7 and Tables 4 and 5.

Table A.2. Four candidate SNRs in the SMC. This is our own measurements shown here for the first time.

\begin{tabular}{|c|c|c|c|c|c|c|c|c|c|}
\hline $\begin{array}{c}\text { Name } \\
\text { MCSNR } \\
(1)\end{array}$ & $\begin{array}{l}\text { Other } \\
\text { name } \\
(2)\end{array}$ & $\begin{array}{c}\text { RA } \\
(\mathrm{J} 2000) \\
(3)\end{array}$ & $\begin{array}{c}\text { Dec } \\
(\mathrm{J} 2000) \\
(4)\end{array}$ & $\begin{array}{c}D_{\text {maj }} \times D_{\text {min }} \\
\left({ }^{\prime \prime}\right) \\
(5)\end{array}$ & $\begin{array}{l}\text { PA } \\
\left({ }^{\circ}\right) \\
(6)\end{array}$ & $\begin{array}{l}D_{\mathrm{av}} \\
(\mathrm{pc}) \\
(7)\end{array}$ & $\begin{array}{c}L_{\mathrm{X}} \\
\left(10^{35} \mathrm{erg} \mathrm{s}^{-1}\right) \\
(8)\end{array}$ & $\begin{array}{c}{[\mathrm{S} \mathrm{II}] / \mathrm{H} \alpha} \\
\text { (9) }\end{array}$ & $\begin{array}{l}\text { Notes } \\
\text { (10) }\end{array}$ \\
\hline J0056-7209 & SNRC1 & 005628.1 & -720942.2 & $340 \times 225$ & 30 & 80.5 & 0.14 & 0.47 & X-ray \& Optical; confirmed as SNR \\
\hline J0057-7211 & N S66D & 005749.9 & -721147.1 & $180 \times 145$ & 45 & 47.0 & 0.17 & - & $\mathrm{X}$-ray \& Radio $\left(\alpha=-0.75 \pm 0.04 ; S_{1 \mathrm{GHz}}=0.0308 \mathrm{Jy}\right)$; confirmed as SNR \\
\hline J0106-7242 & & 010632.1 & -724217.0 & $140 \times 170$ & 0 & 44.9 & - & - & Radio $\left(\alpha=-0.55 \pm 0.02 ; S_{1 \mathrm{GHz}}=0.0236 \mathrm{Jy}\right) ;$ SNR candidate \\
\hline J0109-7318 & SNRC4 & 010943.6 & -731846.0 & $105 \times 105$ & 0 & 30.5 & - & 0.46 & Optical; SNR candidate \\
\hline
\end{tabular}

Notes. Columns (1)-(8) are as in Table A.1. Column (9) is the $[\mathrm{S} \mathrm{II]/H} \alpha$ measured with the WiFeS spectrograph. In Notes (Col. 10) we indicate the waveband in which SNR features are detected and the final status of the source (confirmed or candidate). 
P. Maggi et al.: The supernova remnant population of the Small Magellanic Cloud

Table A.3. X-ray spectral results of SMC SNRs.

\begin{tabular}{|c|c|c|c|c|c|c|c|c|c|c|c|}
\hline \multirow[b]{2}{*}{ MCSNR } & \multicolumn{5}{|c|}{ Component 1} & \multicolumn{5}{|c|}{ Component 2} & \multirow{2}{*}{$\chi^{2} / v\left(\chi_{\text {red }}^{2}\right)$} \\
\hline & $\begin{array}{c}N_{\mathrm{H} \mathrm{LMC}} \\
\left(10^{21} \mathrm{~cm}^{-2}\right)\end{array}$ & $\begin{array}{c}k T \\
(\mathrm{keV})\end{array}$ & $\begin{array}{c}\tau \\
\left(10^{11} \mathrm{scm}^{-3}\right)\end{array}$ & $\begin{array}{c}\mathrm{EM} \\
\left(10^{58} \mathrm{~cm}^{-3}\right)\end{array}$ & Abundances & $\begin{array}{c}N_{\mathrm{H} \mathrm{LMC}} \\
\left(10^{21} \mathrm{~cm}^{-2}\right)\end{array}$ & $\begin{array}{c}k T \\
(\mathrm{keV})\end{array}$ & $\begin{array}{c}\tau \\
\left(10^{11} \mathrm{scm}^{-3}\right)\end{array}$ & $\begin{array}{c}\mathrm{EM} \\
\left(10^{58} \mathrm{~cm}^{-3}\right)\end{array}$ & Abundances & \\
\hline (1) & (2) & (3) & (4) & (5) & (6) & (7) & (8) & (9) & (10) & (11) & (12) \\
\hline J0041-7336 & $1.09_{-0.80}^{+1.04}$ & $0.65_{-0.12}^{+0.09}$ & $2.24_{-0.93}^{+1.30}$ & $4.01_{-0.39}^{+0.96}$ & RD92 & & & - & & & $5788.5 / 5562(1.04)$ \\
\hline $\begin{array}{c}\text { J0046-7308 } \\
*\end{array}$ & $4.06_{-1.13}^{+1.71}$ & $0.60 \pm 0.11$ & $1.01_{-0.40}^{+0.80}$ & $7.71_{-1.64}^{+3.45}$ & $\begin{array}{l}\text { O: } 0.42_{-0.10}^{+0.15} \\
\text { Ne: } 0.45_{-0.10}^{+0.13} \\
\text { Si: } 2.24_{-0.78}^{+1.15}\end{array}$ & & & - & & & $12392.8 / 11672(1.06)$ \\
\hline J0047-7308 & $14.1 \pm 2.20$ & $0.60_{-0.05}^{+0.04}$ & $75.8_{-50.1}^{+424}$ & $13.7_{-0.45}^{+4.87}$ & $\begin{array}{l}\text { Ne: } 3.11_{-0.78}^{+1.10} \\
\text { Mg: } 1.5_{-0.42}^{+0.58}\end{array}$ & & & - & & & $7554.32 / 6219(1.21)$ \\
\hline J0047-7309 & $12.2_{-2.40}^{+1.10}$ & $0.63_{-0.13}^{+0.25}$ & $3.30_{-1.69}^{+5.43}$ & $5.64_{-4.78}^{+4.87}$ & $\begin{array}{c}\text { O: } 8.25_{-4.89}^{+67} \\
\text { Ne: } 7.71_{-3.63}^{+52.3} \\
\text { Mg: } 3.56_{-1.35}^{+4.75}\end{array}$ & & & - & & & 10224.9/8801 (1.16) \\
\hline J0048-7319 & $6.31_{-2.84}^{+3.54}$ & $1.07_{-0.18}^{+0.76}$ & $2.03_{-0.80}^{+4.49}$ & $1.03_{-0.58}^{+0.65}$ & $\begin{array}{c}\text { Mg: } 2.39_{-0.92}^{+1.82} \\
\text { Fe: } 1.17_{-0.49}^{+1.25}\end{array}$ & & & - & & & $4596.9 / 3702(1.24)$ \\
\hline J0049-7314 ${ }^{(a)}$ & $4.39_{-2.02}^{+2.13}$ & $0.52_{-0.14}^{+0.15}$ & $\mathrm{CIE}$ & $3.16_{-1.64}^{+3.63}$ & RD92 & 4.39 & $0.91 \pm 0.03$ & CIE & $7.28_{-0.99}^{+1.25}$ & pure $\mathrm{Fe}$ & 2337.0/2148 (1.09) \\
\hline J0051-7321 ${ }^{(a),(b)}$ & $0.56_{-0.09}^{+0.17}$ & $0.21 \pm 0.01$ & CIE & $171_{-33.6}^{+22.4}$ & RD92 & 0.56 & $0.73_{-0.10}^{+0.16}$ & $1.95_{-0.69}^{+0.93}$ & $12.4_{-2.15}^{+5.26}$ & $\begin{array}{c}\text { Ne: } 1.74_{-0.52}^{+0.77} \\
\text { Mg: } 0.93_{-0.32}^{+0.46} \\
\text { Si: } 2.13_{-0.77}^{+1.14}\end{array}$ & 2124.4/1576 (1.35) \\
\hline J0052-7236 & $3.72_{-1.60}^{+3.35}$ & $0.38_{-0.16}^{+0.66}$ & $0.71_{-0.44}^{+3.79}$ & $27.4_{-24.6}^{+308}$ & $\begin{array}{c}\text { O: } 0.12_{-0.07}^{+0.23} \\
\text { Ne: } 0.24_{-0.12}^{+0.55} \\
\text { Mg: } 0.19(<0.45) \\
\text { Fe: } 0.11_{-0.07}^{+0.15}\end{array}$ & & & - & & & 9101.2/8265 (1.10) \\
\hline $\mathrm{J} 0058-7217^{(c)}$ & $8.49_{-2.49}^{+2.75}$ & $0.37_{-0.09}^{+0.26}$ & $6.31_{-4.61}^{+4.94}$ & $28.5_{-22.1}^{+60.5}$ & $\begin{array}{c}\text { O: } 0.26_{-0.14}^{+0.45} \\
\text { Ne: } 0.30_{-0.15}^{+0.42} \\
\text { Mg: } 0.27_{-0.15}^{+0.21} \\
\text { Fe: } 0.02_{-0.02}^{+0.04} \\
\text { O: } 0.30_{-0.06}^{+0.03}\end{array}$ & & & - & & & $41890.5 / 35193(1.19)$ \\
\hline J0059-7210 & $0.12(<0.21)$ & $0.66 \pm 0.03$ & $7.08_{-1.48}^{+1.99}$ & $25.9_{-2.15}^{+2.37}$ & $\begin{array}{c}\text { Ne: } 0.47_{-0.07}^{+0.08} \\
\text { Mg: } 0.25 \pm 0.05 \\
\text { Fe: } 0.16 \pm 0.02\end{array}$ & & & - & & & $12096.5 / 10380(1.17)$ \\
\hline J0100-7133 & $1.96_{-1.40}^{+3.16}$ & $0.49_{-0.16}^{+0.14}$ & $3.58_{-1.80}^{+11.3}$ & $3.28_{-0.99}^{+4.96}$ & Ne: $0.43 \pm 0.21$ & & & - & & & $3722.0 / 3610(1.03)$ \\
\hline $\mathrm{J} 0103-7209^{(d)}$ & $1.89_{-0.91}^{+1.35}$ & $1.0_{-0.20}^{+0.35}$ & $0.66_{-0.35}^{+0.58}$ & $1.97_{-0.40}^{+0.45}$ & Ne: $0.50_{-0.13}^{+0.15}$ & & & - & & & $12282.7 / 10266(1.20)$ \\
\hline $\mathrm{J} 0103-7247^{(e)}$ & $1.60_{-0.97}^{+1.33}$ & 1.3 & 0.07 & $0.93_{-0.35}^{+0.45}$ & RD92 & & & - & & O: $1.23_{-0.25}^{+0.35}$ & 1877.4/1642 (1.14) \\
\hline $\mathrm{J} 0105-7223^{(a)}$ & $0.82 \pm 0.01$ & $0.19 \pm 0.01$ & $\mathrm{CIE}$ & $448 \pm 51.7$ & RD92 & 0.82 & $0.38 \pm 0.01$ & CIE & $85.3_{-20.7}^{+26.3}$ & $\begin{array}{c}\text { Ne: } 1.64_{-0.57}^{+0.53} \\
\text { Mg: } 0.94_{-0.20}^{+0.28} \\
\text { Fe: } 0.13 \pm 0.06\end{array}$ & $8606.8 / 7123(1.21)$ \\
\hline J0105-7210 $0^{(a)}$ & $1.74_{-0.98}^{+1.41}$ & $0.68_{-0.11}^{+0.20}$ & CIE & $4.44_{-0.78}^{+1.72}$ & RD92 & 1.74 & $0.78_{-0.10}^{+0.07}$ & CIE & $1.88_{-0.58}^{+0.63}$ & pure $\mathrm{Fe}$ & $3722.6 / 3610(1.03)$ \\
\hline J0106-7205 ${ }^{(a)}$ & $3.76_{-0.45}^{+1.10}$ & $0.72_{-0.08}^{+0.07}$ & $\mathrm{CIE}$ & $9.95_{-1.51}^{+1.59}$ & RD92 & 3.76 & $1.0 \pm 0.03$ & CIE & $7.88_{-0.95}^{+1.03}$ & pure $\mathrm{Fe}$ & $3816.96 / 311$ (1.23) \\
\hline $\mathrm{J} 0127-7333^{(f)}$ & $10.2_{-8.10}^{+12.1}$ & $0.19_{-0.01}^{+0.02}$ & $\mathrm{CIE}$ & $25.8_{-12.7}^{+19.1}$ & RD92 & & & - & & & 1918.9/1749 (1.10) \\
\hline & & & & & Candidate SNRs & $X$-raye & sion & & & & \\
\hline J0056-7209 & $0.97(<3.10)$ & $0.7_{-0.46}^{+0.89}$ & $0.28_{-0.25}^{+0.79}$ & $1.48_{-0.81}^{+4.21}$ & $\begin{array}{c}\text { O: } 0.12_{-0.06}^{+0.38} \\
\text { Ne: } 0.06(<0.34) \\
\text { Mg: } 0.30(<1.55) \\
\text { Fe: } 0.20_{-0.17}^{+0.63}\end{array}$ & & & - & & & $5204.2 / 4743(1.10)$ \\
\hline J0057-7211 & $0(<0.24)$ & $0.88_{-0.23}^{+0.41}$ & $1.04_{-0.61}^{+0.66}$ & $1.10(<0.28)$ & RD92 & & & - & & & $26563.4 / 22410(1.19)$ \\
\hline
\end{tabular}

Notes. Columns are described in Sect. 4.3.1. ${ }^{(a)}$ Same absorption column in the two components. ${ }^{(b)}$ Only MOS data used. ${ }^{(c)}$ Fit includes the model from Maitra et al. (2015) for the interior PWN. ${ }^{(d)}$ Bright point source AX J0103-722 excised, see Sect. 4.5. ${ }^{(e)}$ Include a power-law $(\Gamma=2.8)$ and thermal parameters $k T$ and $\tau$ fixed to the values from joint XMM-Newton/Chandra analysis of Crawford et al. $(2014) .{ }^{(f)}$ Central point source SXP 1062 excised. 


\section{Appendix B: Multiwavelength images, spectra, and local star formation history of SMC SNRs}

In this appendix we show for each SNR information about their stellar environment in the form of CMD and SFH plots (see Sect. 4.7 for details), radio and X-ray spectra, and multiwavelength images. We show ASKAP $1320 \mathrm{MHz}$ contours on colour-coded X-ray images, where the red, green, and blue components are the images in the soft, medium, and hard $\mathrm{X}$-ray band as described in Sect. 2.1. The ASKAP beam size of $16.3^{\prime \prime} \times 15.1^{\prime \prime}$ is indicated by the thick magenta ellipse. We also show X-ray contours on optical emission-line images (MCELS), with bands as on Fig. 1. Radio flux density contours increase in the order white-cyan-magenta-red, with the levels used for the contours tailored for each case and given in the captions. The red, green, and blue X-ray contours are taken from the corresponding X-ray band. On the images a spatial scale of $1^{\prime}$ is shown by the white bar. For clarity, we only show up to one pn (black) and one MOS spectrum (blue), although much more might have been used for spectral analysis (see Sect. 2.1). The sum of all background components is shown has the grey dotted line. The SNR model is shown in magenta by the solid (pn) or dashed (MOS) line. For cases with a second SNR component it is displayed in green. When a contributing point source (X-ray binary, PWN), related to the SNR or not, is included, we show its emission model in cyan. Fit statistics $\left(\chi^{2} / v\right)$ are given on the plot. Residuals are shown in units of standard deviation.
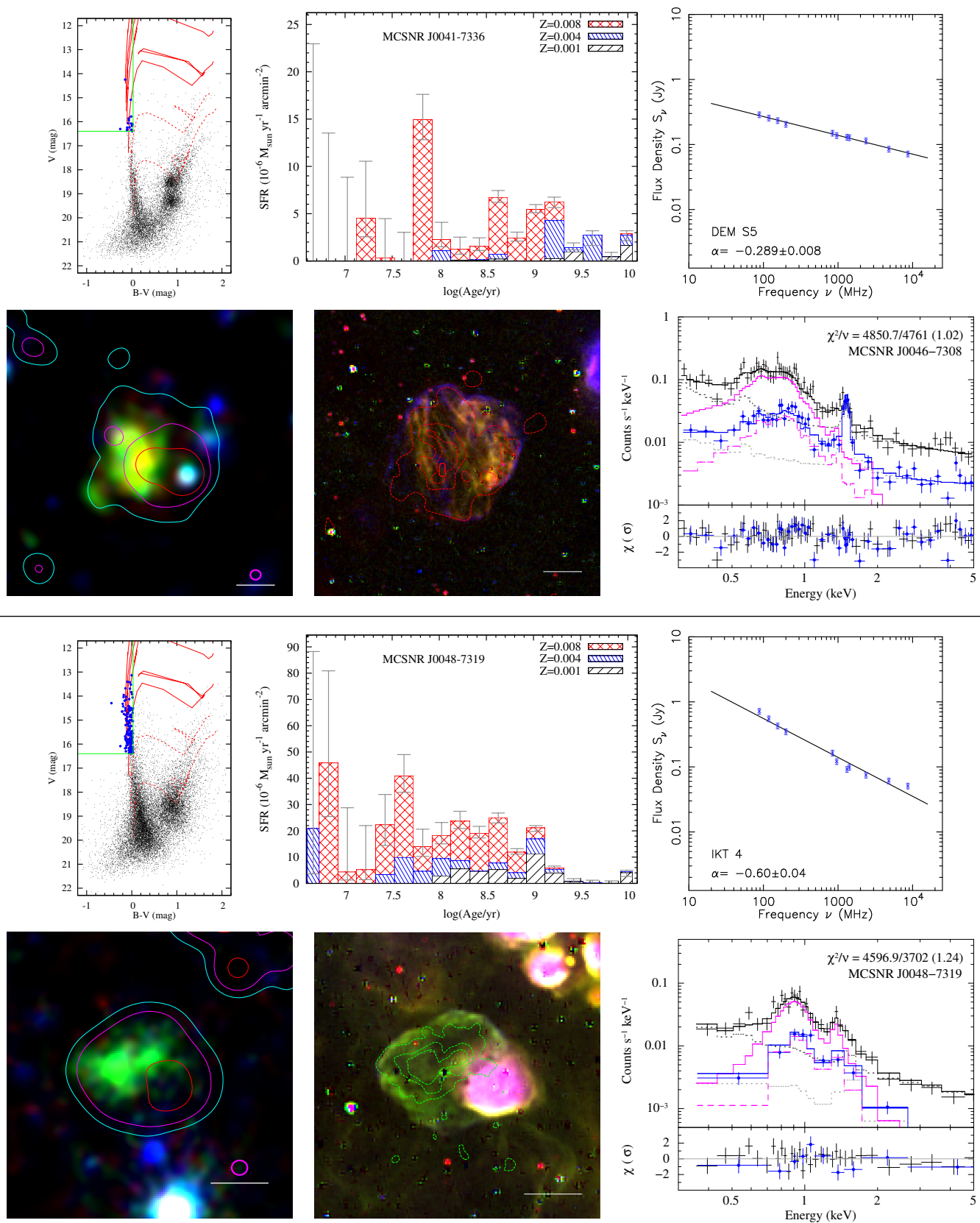

Fig. B.1. Top part: CMD, SFH, and radio-continuum spectrum of MCSNR J0041-7336 (first line), X-ray image with radio contours, optical image with X-ray contours, and X-ray spectrum (left, middle, and right panel of the second line, respectively). The radio flux density levels shown are at $0.1 \mathrm{mJy}$ beam $^{-1}, 0.5 \mathrm{mJy}_{\text {beam }}^{-1}$, and $2 \mathrm{mJy}_{\text {beam }}{ }^{-1}$. Bottom part: same as above for MCSNR J048-7319, with same radio contour levels. 
P. Maggi et al.: The supernova remnant population of the Small Magellanic Cloud
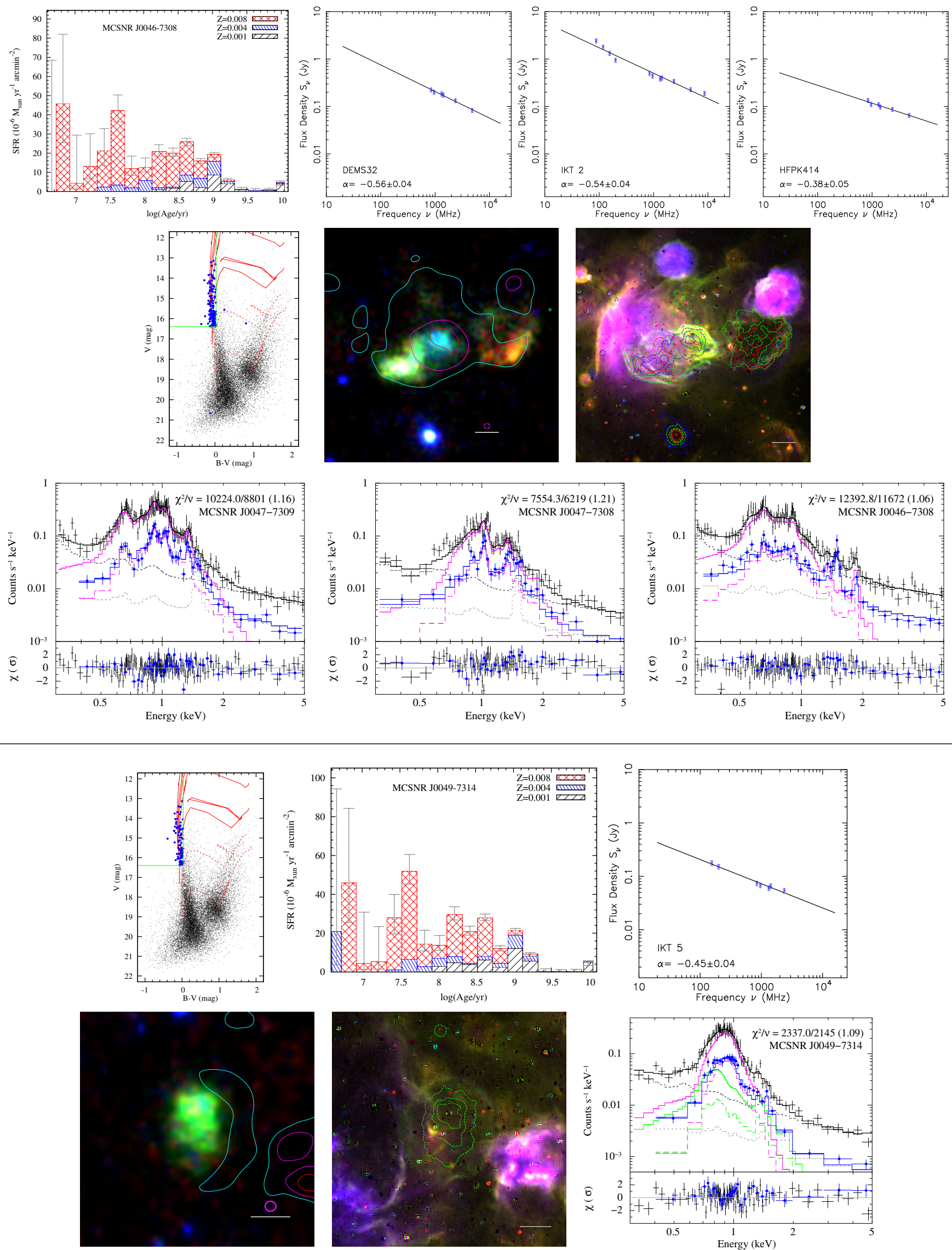

Fig. B.2. Top part: for the three SNRs in the N19 complex (Sect. 4.5.2) we show a single CMD and SFH as they are in the same photometric grid of Harris \& Zaritsky (2004). The three SNRs are shown on the same images, with from left to right (decreasing RA) : MCNR J0047-7309, J0047-7308, J0046-7308; the radio and X-ray spectra are shown in the same order. The radio contour levels are at 1, 3, and $8 \mathrm{mJy} \mathrm{beam}^{-1}$. Bottom part: same as Fig. B.1 for MCSNR J0049-7314, with radio contour levels at 0.5, 1, and $2 \mathrm{mJy} \mathrm{beam}^{-1}$. 
A\&A 631, A127 (2019)
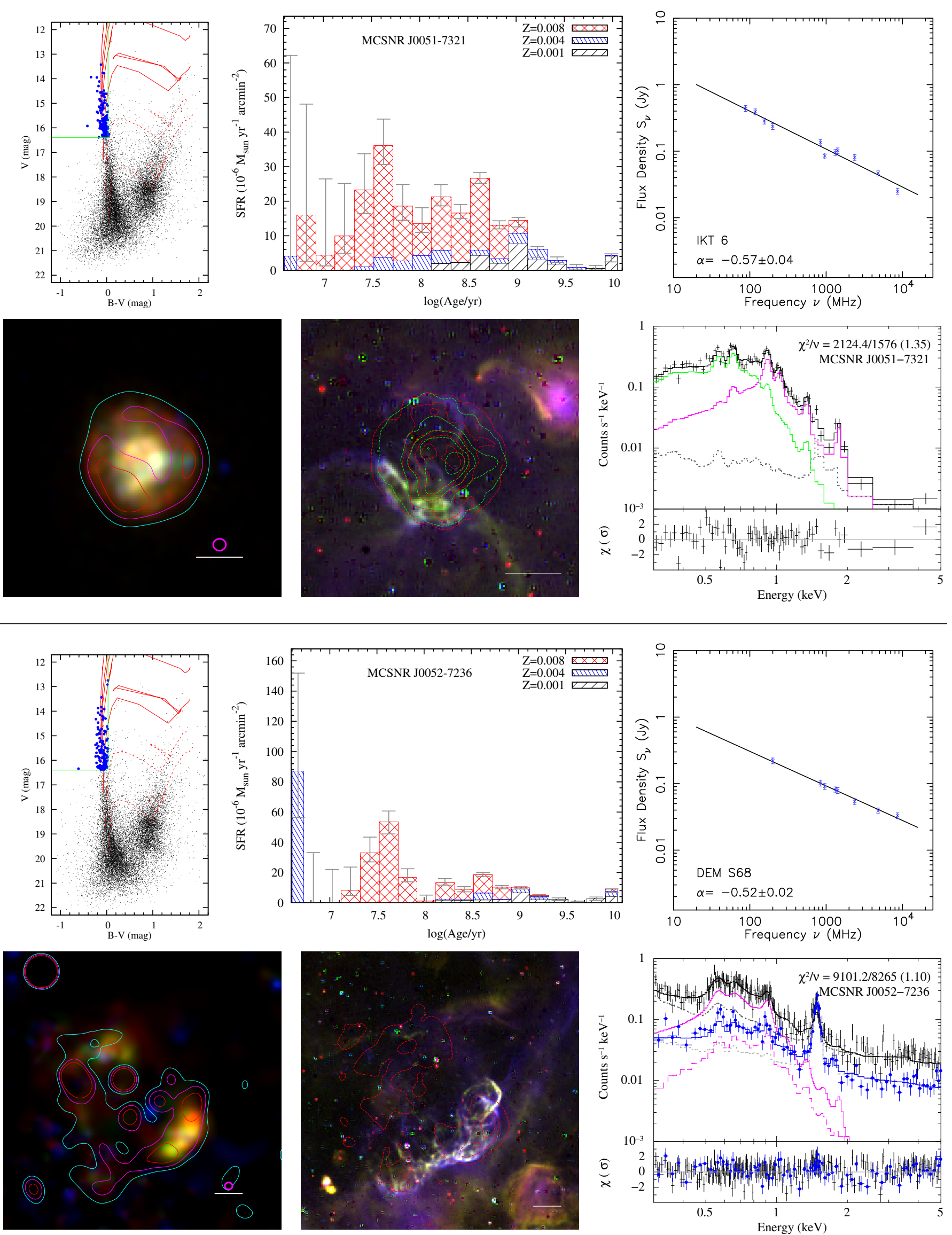

Fig. B.3. Same as Fig. B.1 for MCSNR J0051-7321 (top part), with radio contour levels at 0.5, 1, and $1.5 \mathrm{mJy}^{\text {beam }}{ }^{-1}$, and for MCSNR J0052-7236 with levels at $0.3,0.5$, and $0.8 \mathrm{mJy}_{\text {beam }}^{-1}$ (bottom part). 
P. Maggi et al.: The supernova remnant population of the Small Magellanic Cloud
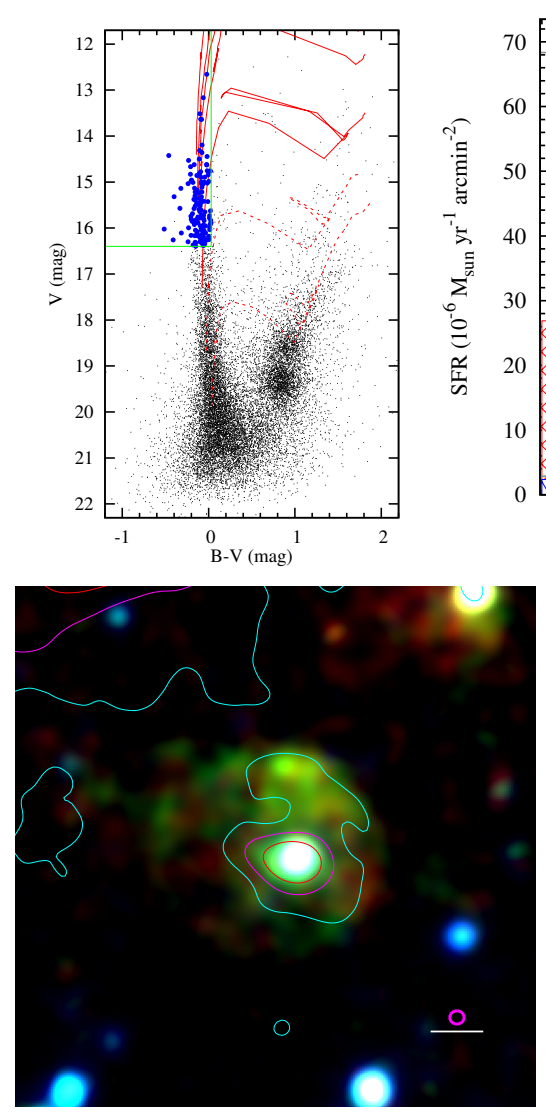
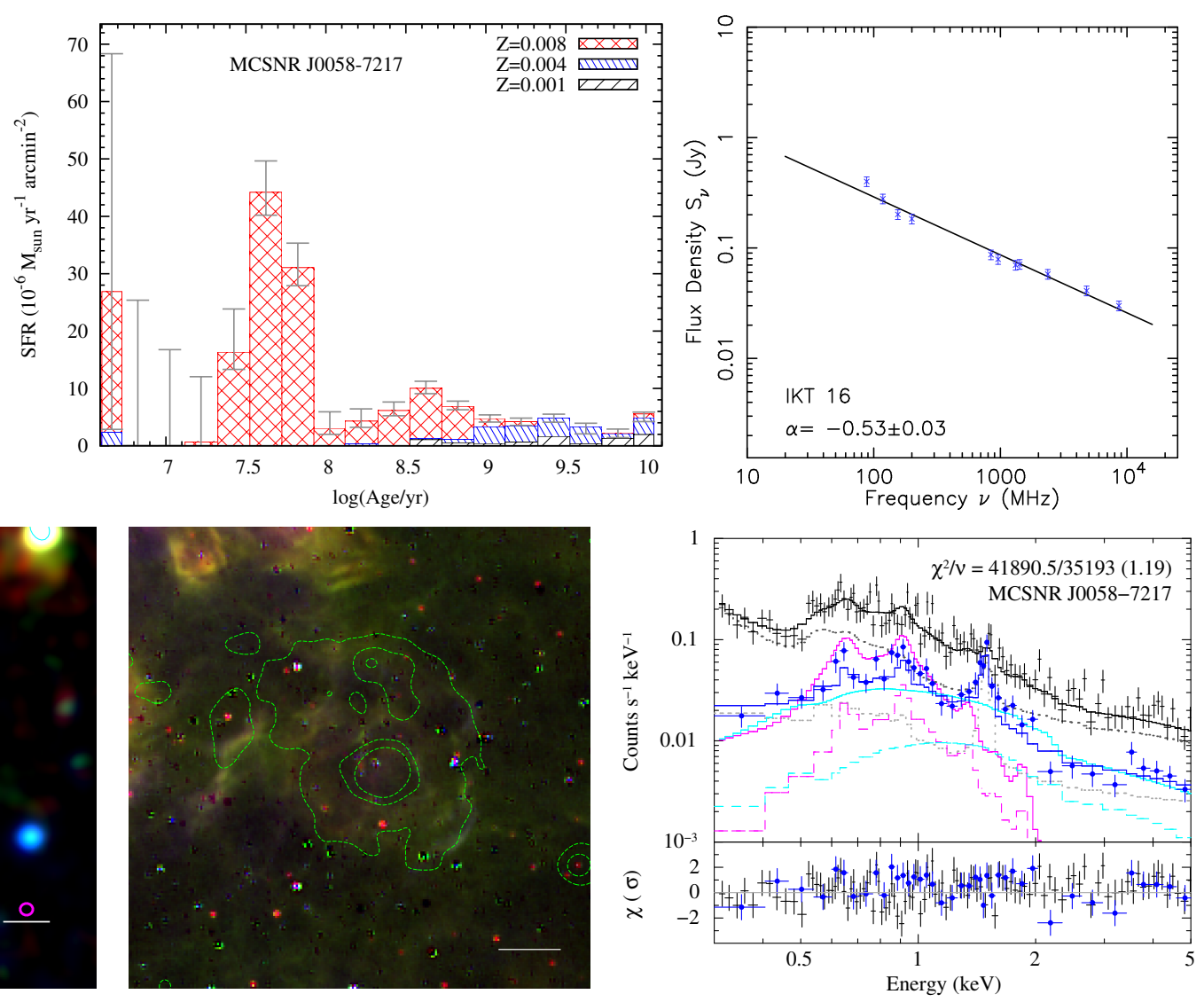

IKT 16
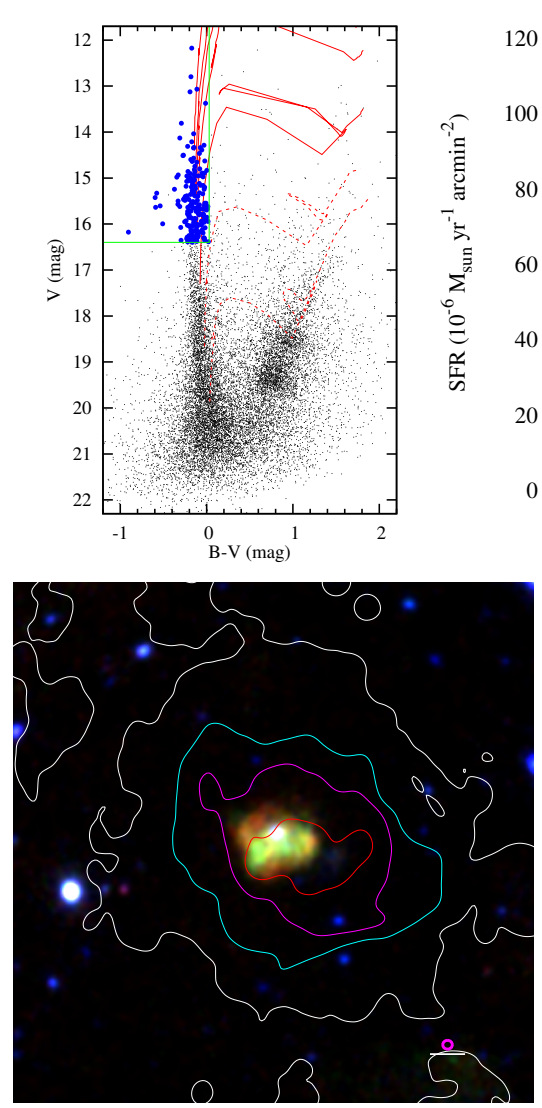
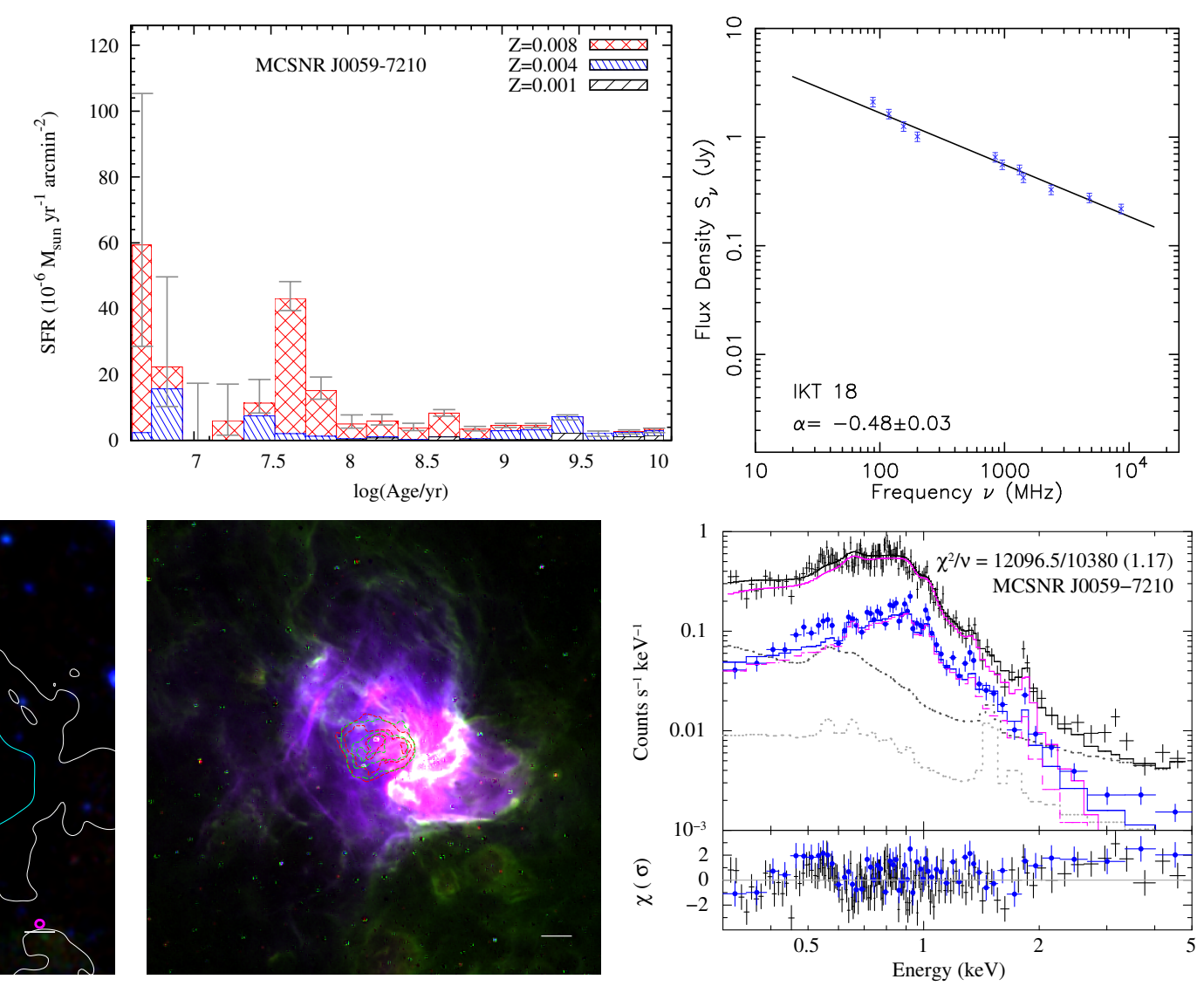

Fig. B.4. Same as Fig. B.1 for MCSNR J0058-7217 (top part), with radio contour levels at $0.15,0.6$, and $1.5 \mathrm{mJy}^{\mathrm{beam}}{ }^{-1}$, and for MCSNR J0059-7210 with levels at $0.15,0.8,2$, and $8 \mathrm{mJy}^{\text {beam }}{ }^{-1}$ (bottom part). 

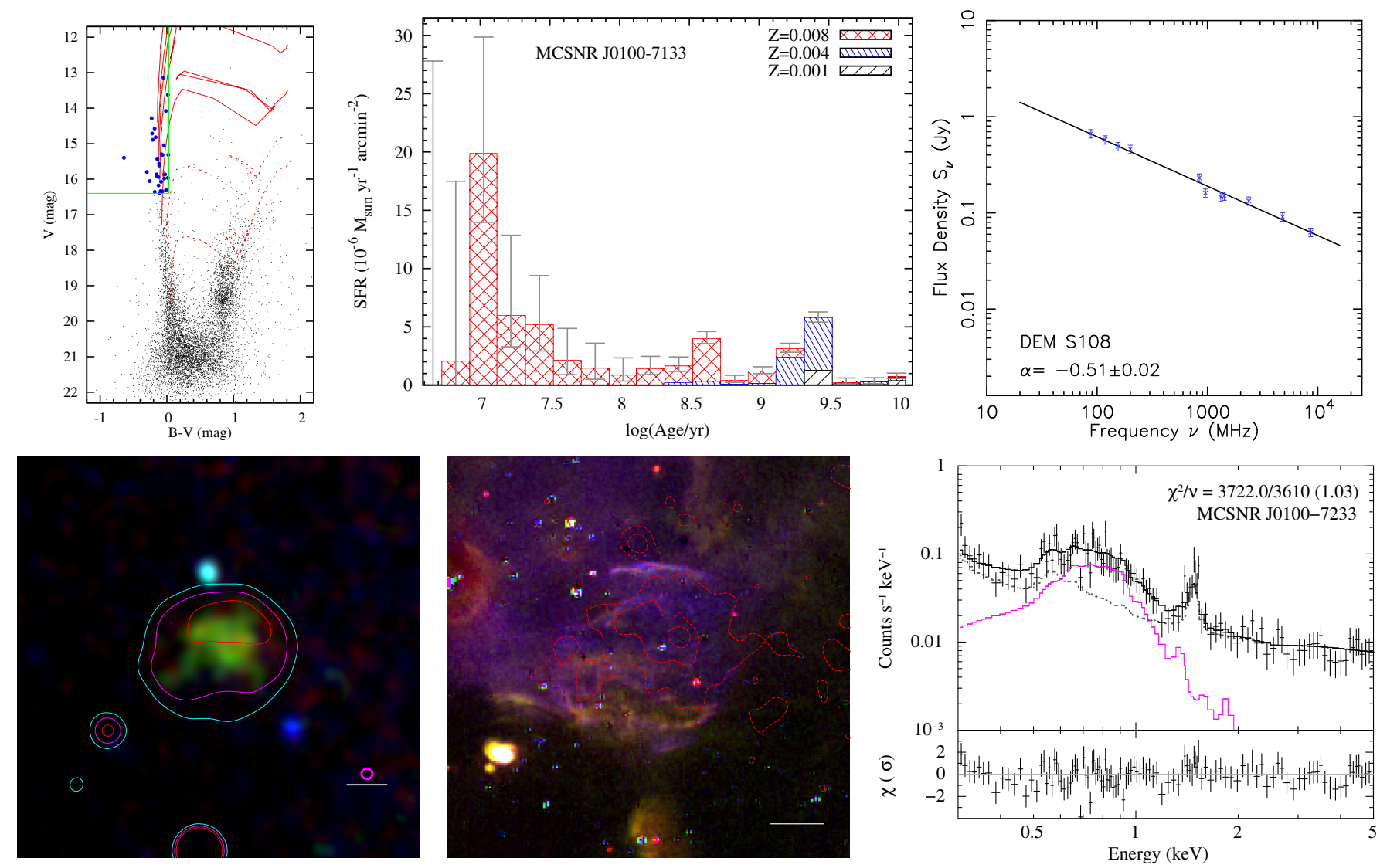
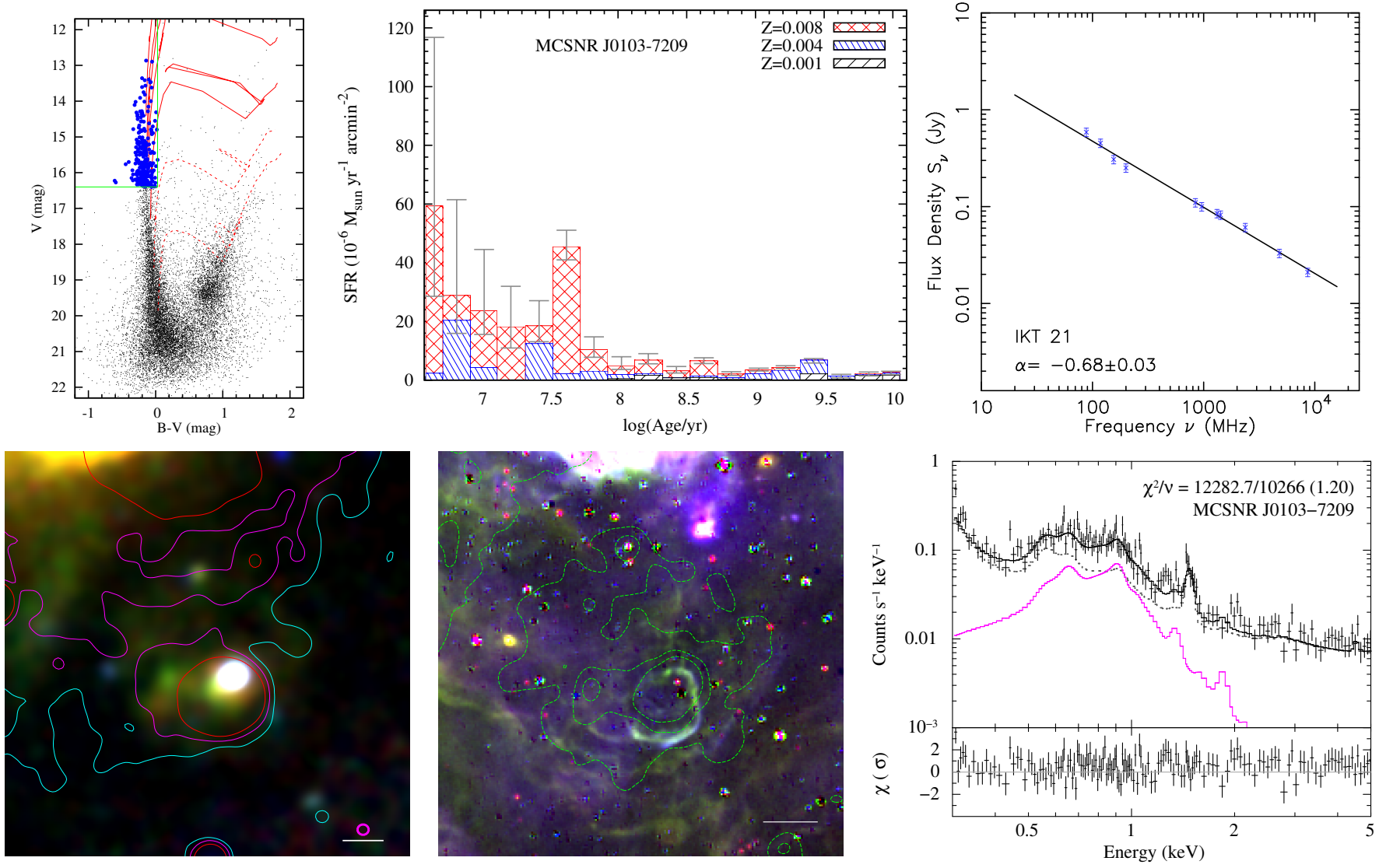

Fig. B.5. Same as Fig. B.1 for MCSNR J0100-7133 (top part), with radio contour levels at $0.3,0.8$, and $1.5 \mathrm{mJy}^{\mathrm{beam}}{ }^{-1}$, and for MCSNR J0103-7209 with levels at 0.15, 0.3, and $0.6 \mathrm{mJy}^{\text {beam }}{ }^{-1}$ (bottom part). 
P. Maggi et al.: The supernova remnant population of the Small Magellanic Cloud
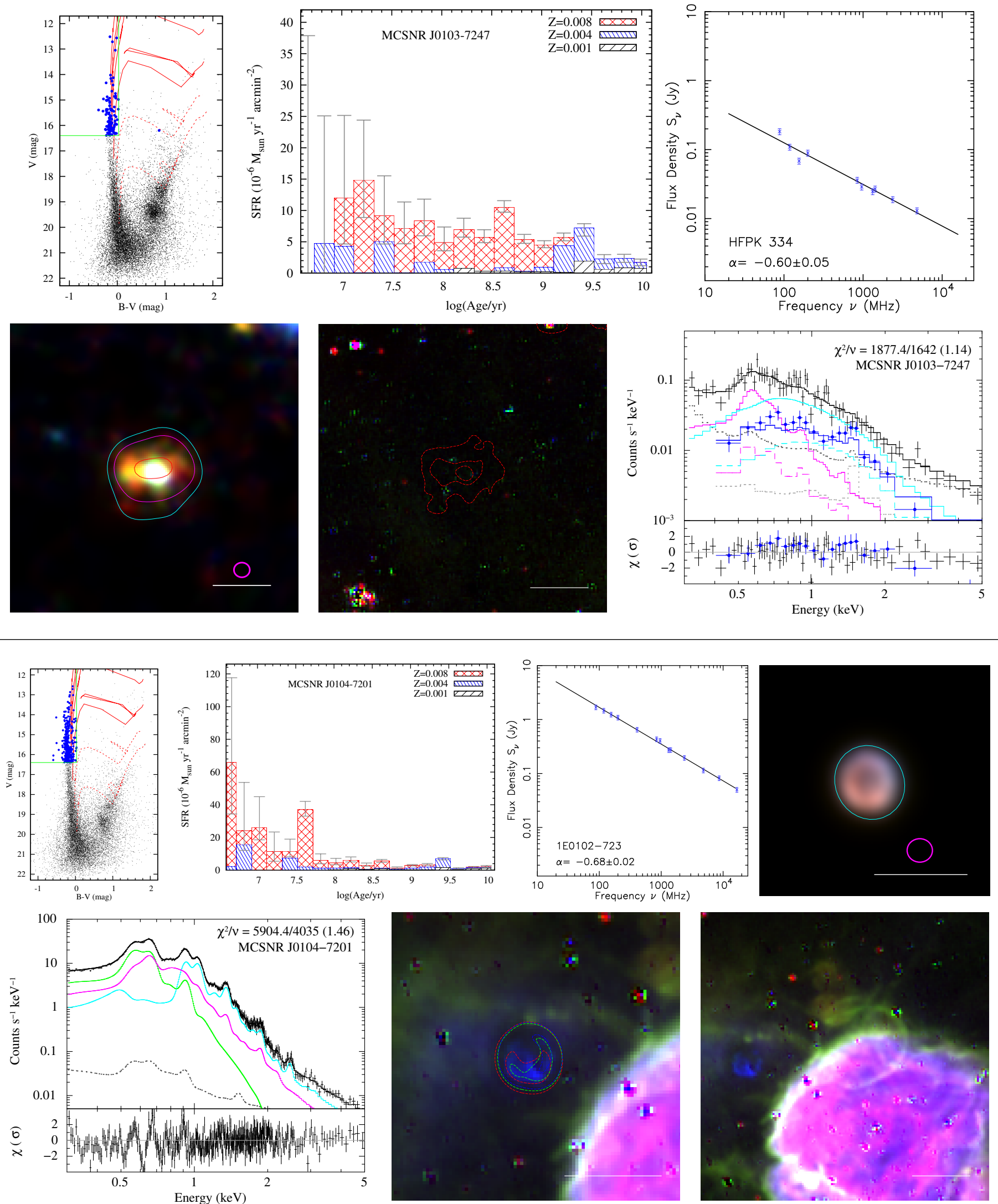

Fig. B.6. Same as Fig. B.1 for MCSNR J0103-7247 (top part), with radio contour levels at 0.3 , 0.6 , and $1.5 \mathrm{mJy}^{\mathrm{m}}$ beam ${ }^{-1}$, and for MCSNR J0104-7201 with one level at $20 \mathrm{mJy}$ beam $^{-1}$ (bottom part). At the bottom right we show the MCELS image of MCSNR J0103-7201, identified by its $\mathrm{H} \alpha$ shell. Its CMD and SFH are essentially the same as that of the neighbouring J0104-7201. 
A\&A 631, A127 (2019)
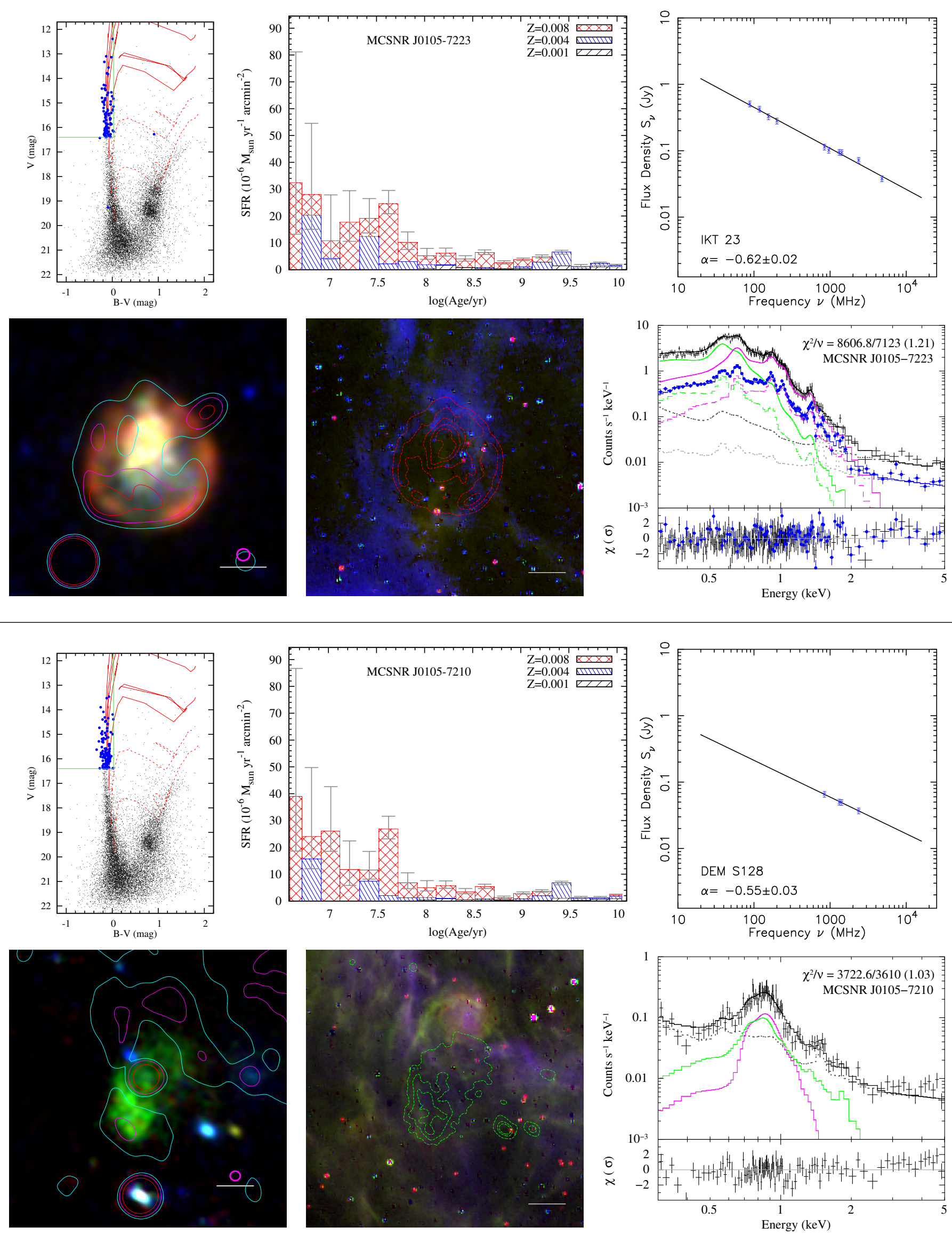

Fig. B.7. Same as Fig. B.1 for MCSNR J0105-7223 (top part), with radio contour levels at 0.3 , 0.5 , and $0.8 \mathrm{mJy}^{\mathrm{beam}}{ }^{-1}$, and for MCSNR J0105-7210 with levels at 0.1, 0.2, and 0.5 mJy beam $^{-1}$ (bottom part). 
P. Maggi et al.: The supernova remnant population of the Small Magellanic Cloud
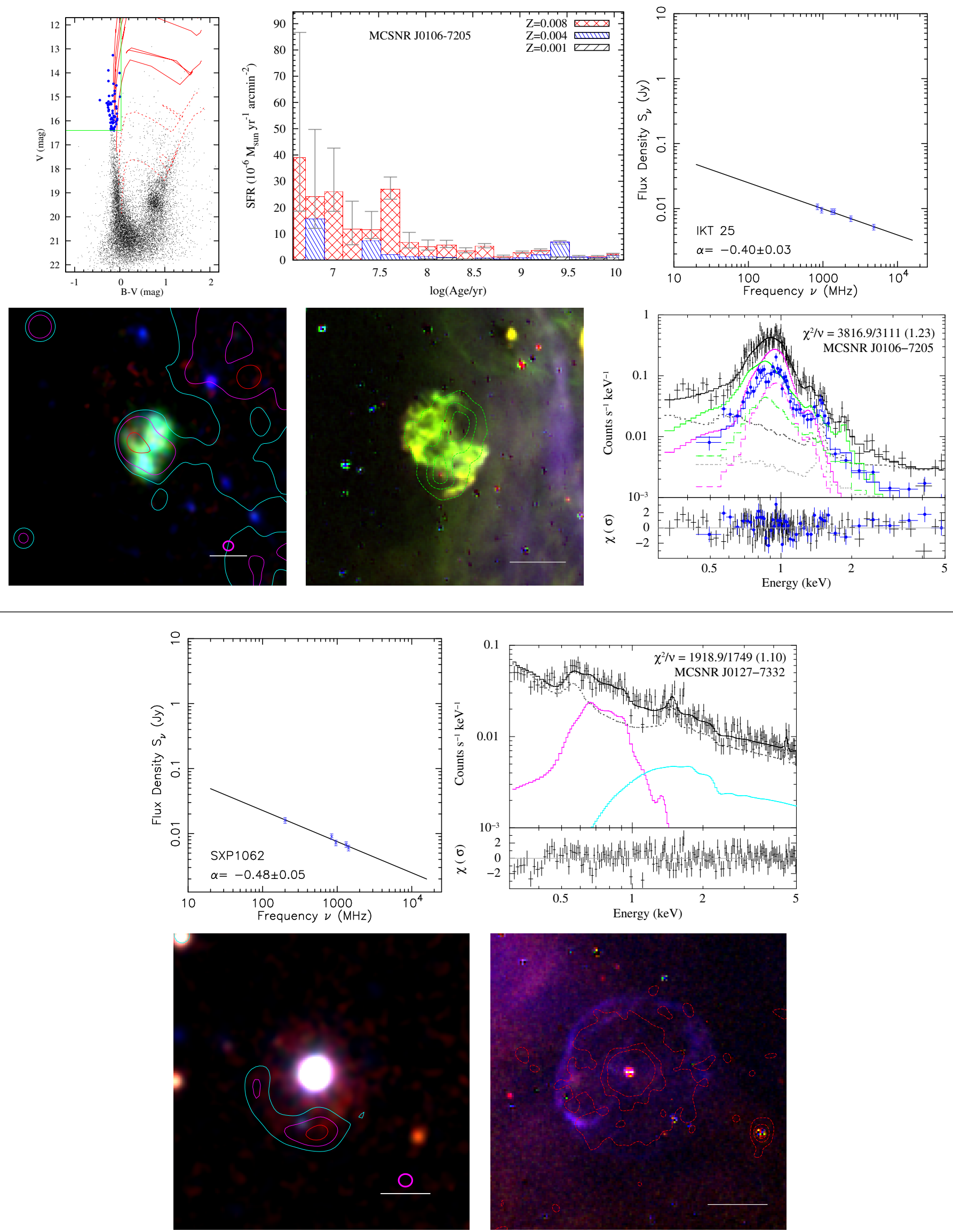

Fig. B.8. Same as Fig. B.1 for MCSNR J0106-7205 (top part), with radio contour levels at 0.1, 0.2, and 0.5 mJy/beam, and for MCSNR J0127-7332 with levels at $0.1,0.15$, and $0.2 \mathrm{mJy} \mathrm{beam}^{-1}$ (bottom part). For the last one, there are neither CMD nor SFH, as it was outside the area covered in the MCPS (Harris \& Zaritsky 2004). 

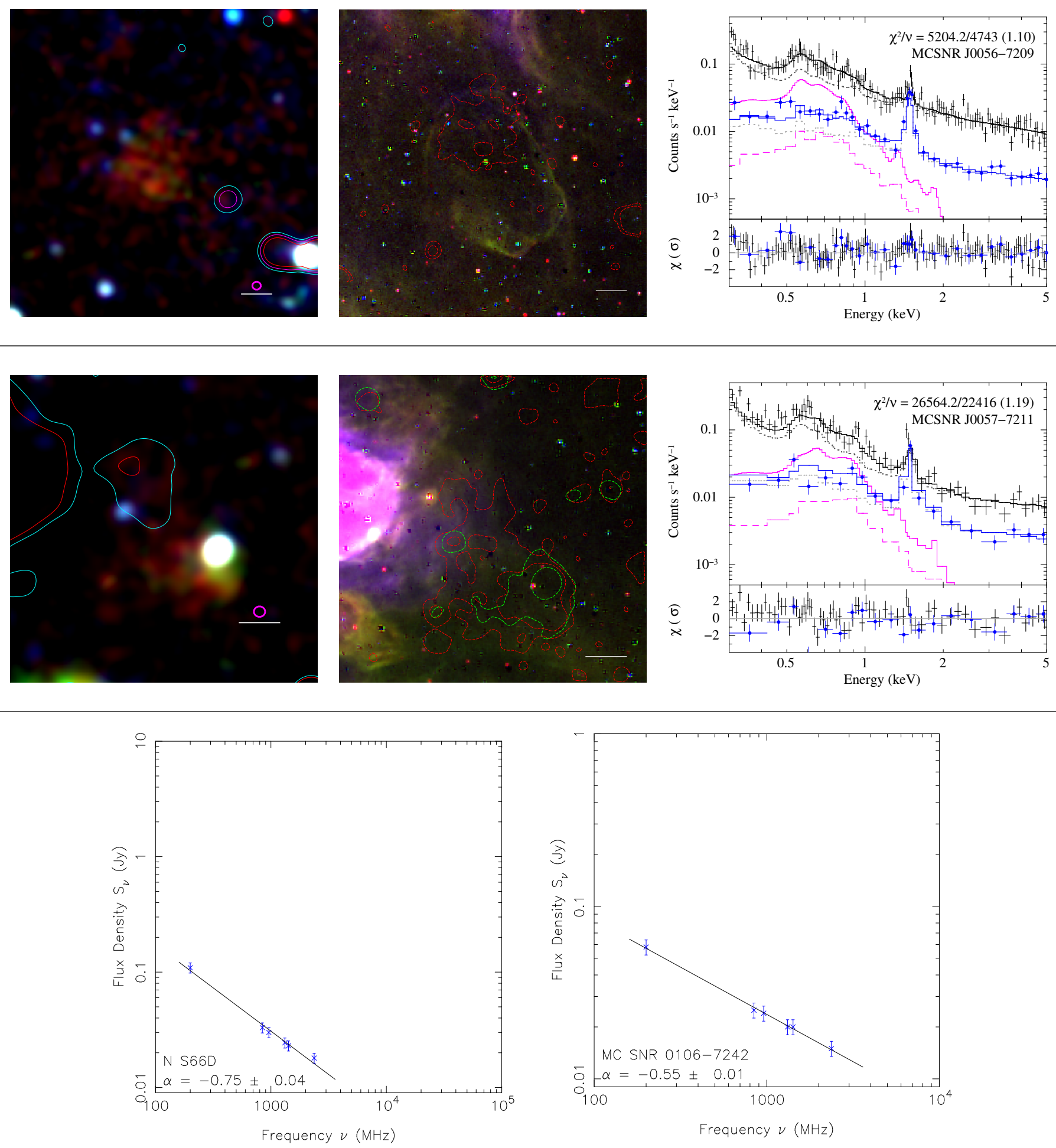

Fig. B.9. Top row: X-ray image of the newly confirmed MCSNR J0056-7209 with radio contours, levels at $0.3,0.8$, and $2 \mathrm{mJy}^{\mathrm{m}}$ beam ${ }^{-1}$; MCELS image with X-ray contours; and X-ray spectrum. Middle row: same as above for the newly confirmed MCSNR J0057-7211. The radio contours are at the 0.3 and $0.5 \mathrm{mJy}_{\text {beam }}{ }^{-1}$ level. Bottom row: radio spectra for the newly confirmed MCSNR J0057-7211 (N S66D) and MCSNR candidate J0106-7242. 